\title{
Self-Organized Formation of Metal-Carbon Nanostructures by Hyperthermal Ion Deposition
}

Dissertation

\author{
zur Erlangung des Doktorgrades \\ der Mathematisch-Naturwissenschaftlichen Fakultäten \\ der Georg-August-Universität zu Göttingen \\ Vorgelegt von \\ Inga Karolin Hannstein, geb. Gerhards \\ aus \\ Brake an der Unterweser
}

Göttingen, 2006 
D7

Referent: Prof. Dr. H. C. Hofsäss

Koreferent: PD Dr. M. Seibt

Tag der Mündlichen Prüfung: 26. April 2006 


\section{Contents}

1 Introduction 3

1.1 The Qualities of Metallic Particles in an Amorphous Carbon Matrix . 5

1.2 Overview of the Status of Research $\ldots \ldots \ldots \ldots \ldots$

1.3 Examples of Self-Organization . . . . . . . . . . . . . . . . 12

2 The Base Materials $\quad 15$

2.1 Carbon . . . . . . . . . . . . . . . . . . . . . . 15

$2.2 \quad$ Metals of the Copper Group and Iron . . . . . . . . . . . . 18

2.3 Structural Properties of Small Metal Clusters . . . . . . . . . . . 20

2.4 Nucleation and Growth of Thin Metal Films on Carbon Substrates . 22

3 Film Growth 26

$3.1 \quad$ Mass Selective Ion Beam Deposition (MSIBD) . . . . . . . . . . . 26

3.1 .1 The MSIBD Setups ADONIS and MR. STRINGER . . . . . . . 26

\begin{tabular}{|ll}
3.2 & Reactive Magnetron Sputtering Deposition (R-MSD) \\
\hline
\end{tabular}$\ldots \ldots \ldots$

3.3 MSIBD versus R-MSD Processes $\ldots \ldots \ldots \ldots \ldots$

3.4 Processes during the Deposition of Hyperthermal Species . . . . . . . 31

\begin{tabular}{|lll}
4 & Characterization Techniques & 39
\end{tabular}

4.1 Transmission Electron Microscopy . . . . . . . . . . . . . . . . . . . . 39

4.2 Rutherford Backscattering Spectroscopy _. . . . . . . . . . . . . 43

4.3 Raman Spectroscopy $\ldots \ldots \ldots \ldots \ldots$

$4.4 \quad$ X-Ray Diffraction . . . . . . . . . . . . . . . . . . . . . . . . . 48

$4.5 \quad$ Auger Electron Spectroscopy Depth Profiling. . . . . . . . . . . . . 49

5 Review of Previous Work 51

5.1 Diffusion in Amorphous Carbon . . . . . . . . . . . . . . . . . . 51

$5.2 \quad$ Amorphous Carbon Containing Copper . . . . . . . . . . . . . . 52

5.3 Amorphous Carbon Containing Silver . . . . . . . . . . . . . 55

$6 \quad$ Spontaneous Formation of Multilayers Reported in the Literature 58

$6.1 \quad$ Experiments by $\mathrm{Wu}$ and Ting $\ldots \ldots \ldots \ldots \ldots \ldots$

6.1 .1 Experimental Results . . . . . . . . . . . . . . . . . . 58

6.1 .2 Catalysis Model for Multilayer Formation . . . . . . . . 59 
6.2 Experiments by Corbella and Co-workers . . . . . . . . . . . 60

$6.2 .1 \quad$ Experimental Results . . . . . . . . . . . . . . . . . 60

6.2 .2 Cahn-Hillard Based Spinodal Decomposition Model . . . . . . 61

6.3 Experiments by He and Co-workers . . . . . . . . . . . . . . . 62

$6.3 .1 \quad$ Experimental Results . . . . . . . . . . . . . . . . . . 63

6.3.2 Combined Cahn-Hillard/Ion Beam Induced Segregation Model 64

7 Morphology of MSIBD-Grown a-C:Me films $\quad 66$

$7.1 \quad$ a-C:Cu Film Morphology Dependence on $\mathrm{Cu}^{+}$Ion Energy $\ldots . . .66$

$7.2 \quad$ Amorphous Carbon Containing Gold $\ldots \ldots \ldots \ldots$

7.3 Amorphous Carbon Containing Iron . . . . . . . . . . . . . . . 77

7.4 Summary and Discussion $\ldots \ldots \ldots \ldots$. . . . . . . . . . . 81

8 Structure Formation Model $\quad 84$

8.1 The Uniform Cluster Distribution of a-C:Cu films . . . . . . . . . . 85

8.2 Surface Segregation and Multilayer Formation . . . . . . . . . . 86

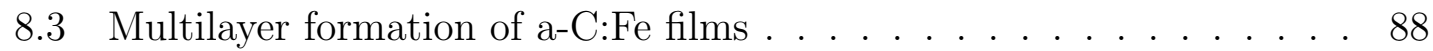

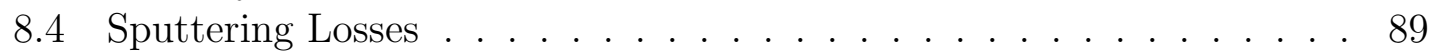

8.5 Estimation of the Multilayer Period for Metal Surface Segregation . . 90

8.6 Estimation of the Multilayer Period for Carbide Forming Metals . . . 92

8.7 Predictions $\ldots \ldots \ldots \ldots \ldots \ldots$

8.8 Comparison of the Multilayer Formation Models . . . . . . . . . . 94

$\begin{array}{lll}9 & \text { Magnetron Sputtered Films } & 96\end{array}$

9.1 Magnetron Sputtered a-C:Cu _ . . . . . . . . . . . . . . . . 97

$9.2 \quad$ Magnetron Sputtered a-C:Fe . . . . . . . . . . . . . . . . . . . . 101

9.2 .1 Variation of the Gas Flow Ratio . . . . . . . . . . . . 102

9.2 .2 Variation of the Target-Substrate Distance. . . . . . . . . . 108

9.2 .3 Variation of RF Power . . . . . . . . . . . . . . . . . . . . 112

9.3 Summary and Discussion . . . . . . . . . . . . . . . . . . . 113

10 Final Discussion and Conclusion 116

\begin{tabular}{ll}
\hline References & 119
\end{tabular} 


\section{Chapter 1}

\section{Introduction}

The need for even smaller and more efficient electronic devices or more sturdy materials has given rise to a vast number of research activities, especially in the field of nanoscience. As quantum effects come into play for nanoscale objects, and the surface to volume ratio becomes eminently large, a number of interesting new material properties arise. These properties may be tailored to meet certain needs, provided the growth processes are well understood.

This work focuses on the investigation of the mechanisms driving the formation of various morphologies of metal-containing amorphous carbon films. In the course of the experiments, an intriguing variety of different morphologies was found: in amorphous carbon films containing either copper or-under certain conditionsgold (a-C:Cu and a-C:Au), metallic precipitates with diameters in the order of a few nanometres are homogeneously distributed; silver-containing amorphous carbon (a-C:Ag) decomposes and the silver accumulates at the surface of a silver-depleted amorphous carbon film; and films containing either gold or iron (a-C:Au or a-C:Fe) may show a structure of alternating layers of more and less densely distributed metallic nanocrystals. Such a self-organized formation of multilayers was found for the first time in the course of this work and presented at nearly the same time as similar studies by $\mathrm{Wu}$ and Ting [1]. It is the aim of this thesis to achieve a better understanding of the evolution of these different morphologies.

Metallic particles of nanometre size embedded in dielectric, and in particular diamond-like, matrices have a number of properties that can make these materials suitable for various applications. These include an interesting linear and non-linear optical behaviour, chemical inertness of the matrix, very good tribological performance, and, where applicable, magnetic properties. Section 1.1 gives a broader 
overview of the promising application potential of such materials. The recent research activities on the structure and structure formation mechanisms are discussed in Section 1.2 .

At present, nanoscale device fabrication is mostly limited to lithographic techniques, which are rather expensive and time-consuming. Therefore, self-organization effects are both technically and scientifically appealing. A range of different mechanisms that are known and used at present are summarized in Section 1.3. However, it will be shown further down that non of these mechanisms are able to account for the morphologies found in this thesis.

Chapter 2 is an introduction to the materials this thesis deals with: the class of interesting carbon materials has grown immensely in the recent past. The two basic carbon phases, graphite and diamond, and especially the matrix material of this work, amorphous carbon, are introduced in Section 2.1. Four different metals were incorporated into the films: the three non-carbide forming metals of the copper group, as well as the magnetic and carbide-forming metal iron. Their chemical properties and interactions with carbon are described in Section 2.2. The structural properties of small metal clusters have turned out to play a major role in the parameters influencing the structure of the films. An overview of experimental and theoretical considerations is given in Section 2.3. Furthermore, the film formation mechanisms of metals on an amorphous carbon surface will help understand the structure of the multilayers. A summary is given in Section 2.4.

The two film growth techniques used in this thesis are mass selective ion beam deposition and reactive magnetron sputter deposition. Both are based on the deposition of particles of energies exceeding thermal energies (a few to a few hundred eV), a process also called deposition of hyperthermal species. The two methods are introduced in Chapter 3 with special emphasis on the setups. A comparative discussion of the growth parameters of these two techniques is given. Furthermore, in Section 3.4, the processes provoked by energetic ions in matter are described, as these are the basic mechanisms that have to be considered for the understanding of the evolution of the different film morphologies. The following Chapter 4 describes the characterization techniques implemented for the analysis of the film composition and nanostructures.

Chapter 5 gives a summary of preceding studies on diffusion of foreign atoms in amorphous carbon as well as the results for copper- and silver-containing amorphous carbon films. 
A self-organized multilayer structure similar to the results of this work for the gold- and iron-containing amorphous carbon films was also found by three other groups [1, 2, 3. Their experimental results and models are described in Chapter 6.

Chapter 7 presents the results of the structural analysis of the films grown by mass selective ion beam deposition. Sections 7.1 through 7.3 give the results of an ion energy dependent analysis of the copper-containing films and the investigation of two further systems: gold and iron incorporated in amorphous carbon films. Including the results presented in Chapter 5, three different structures were found: a homogeneous cluster distribution for the a-C:Cu films, surface accumulation for the a-C:Ag films, and self-organized cluster layers separated by amorphous material for the a-C:Au and a-C:Fe films. An attempt to model the formation of the different structures is given in Chapter 8. This includes an estimation of the periodicities of the multilayers found for a-C:Au and a-C:Fe films, taking into account the structural properties of small clusters of the different metals as well as the influence of the ion impact. A comparison with the models of Wu and Ting [1], Corbella and co-workers [2], and He and co-workers [3] is given in Section 8.8

In order to investigate whether the multilayer formation processes are similar or driven by the same parameters for both magnetron sputter deposition and mass selective ion beam deposition, magnetron-sputtered a-C:Cu and a-C:Fe films were grown. The results are presented in Chapter 9 .

The thesis closes with a final discussion, conclusion and an outlook to future research.

\subsection{The Qualities of Metallic Particles in an Amorphous Carbon Matrix}

Metallic particles embedded in dielectric matrices bear useful linear and non-linear optical properties [4]. These properties have to be attributed to the limited size of the particles, where surface-to-volume effects and quantum effects come into play. In a particle of nanometre size, the electron gas is spatially extremely confined in comparison to its bulk extension. For example correlation lengths or mean free paths of the respective bulk material are much larger than the diameter of the cluster [4, 5]. The optical properties reflect the structure and the dynamics of the delocalized electrons. The more strongly bound electrons and the core form the ionic background for the electron gas. In the case of alkali metals, there is no further influence of 

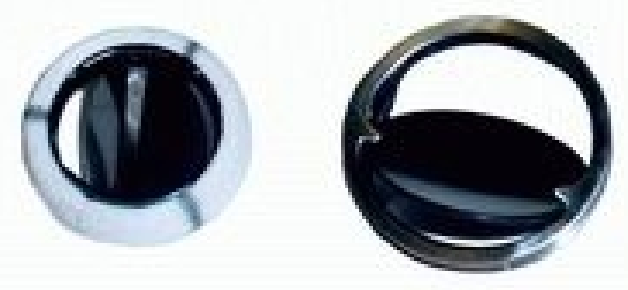

Figure 1.1: Artificial heart valves coated with diamond-like carbon (from [19]). Even better performance may be obtained by introducing metals into medical implants and thus achieving an antibacterial behaviour [18.

excitations in the core levels on the electron gas. However, for transition and noble metals, core level excitation energies are of the order of a plasmon resonance. This also holds true for II-VI and III-V semiconductor clusters [6, 7]. For noble metal nanoparticles, the third-order non-linear susceptibility $\chi^{(3)}$ is strongly enhanced up to $\sim 2.5 \times 10^{-6}$ esu around the plasmon resonance with response times in the picosecond range [8, 9]. These properties make a metal cluster/dielectric host material a promising candidate for optical devices, for instance optical switches [10]. A good overview of linear and non-linear optical properties is given in Reference [11].

Another broad field of application derives from the excellent tribological properties of diamond-like carbon thin films. These may be further advanced when metal particles are embedded in the film. Here, one major aim is the reduction of compressive stress. The compressive stress is typical of most diamond-like materials and limits the film thickness [12,13]. The incorporation of metals provides the possibility to relieve some of this internal stress. Although the $\mathrm{sp}^{3}$ bonding content of the carbon matrix decreases with increasing metal content ([14], this work), the hardness and Young's modulus may remain at rather high values [15]. An approach to achieve the best performance is to grow metal-containing amorphous carbon films with a gradient metal concentration, yielding a higher metal content at the substrate/film interface that decreases towards the surface. This is, for example achieved by alternate laser ablation of carbon and the respective metal from a rotating graphite target with metal strips attached to its surface. During deposition, the laser spot is moved from its position close to the centre towards the edge of the target [16, 17].

Together with the chemical inertness and the resulting biocompatibility, the good tribological properties of diamond-like carbon lead to two biomedical applications: as a coating for either blood contacting implants, such as heart valves or stents, or prosthetic joint replacements [18]. The former are already commercially available (see Figure 1.1). In various in vitro and in vivo experiments, diamond-like carbon coated medical implants feature a high albumin/fibrinogen ratio, a low number of 
platelets adhering to the surface, a decreased platelet activation, and a decreased tendency of thrombus formation, due to their excellent haemocompatibility. The introduction of certain toxic elements, such as copper, silver, or vanadium results in an antibacterial effect.

Another field of application arises, when the metal incorporated in the diamondlike carbon matrix offers magnetic properties. FePt and CoPt nanocrystals may exhibit a large uniaxial magnetocrystalline anisotropy of $K_{u} \cong 7 \times 10^{6} \mathrm{~J} / \mathrm{m}^{3}$ if present in the face centred tetragonal phase. The $K_{u}$ value together with the particle volume determines the magnetic stability of individual particles. Therefore, small CoPt and FePt particles may be suitable for future ultra-high density recording media [20, 21, 22]. In order to decrease the exchange interaction, it is expedient to separate the clusters by a non-magnetic matrix material, for example carbon $[23,24,25]$. Diamond-like carbon films are already used as coatings of commercially available hard disk drives [26], and progress is being made towards ever thinner amorphous carbon films with high $\mathrm{sp}^{3}$ bonding contents in order to further decrease the spacing between the disc and the read/write head and hence increase the recording density [27]. A further progress would be to grow CoPt or FePt particles within an amorphous carbon matrix of diamond-like quality.

\subsection{Overview of the Status of Research on Metal- Containing Amorphous Carbon}

Over the past two decades, there has been a considerable amount of research about the structure and structure formation of metal-containing diamond-like carbon thin films, which is, of course, mainly due to the promising potential in the various fields of application mentioned above. There are a number of methods that are used for the preparation of carbon films containing metal nanoparticles, including pulsed laser deposition [28, 29], integrated nanocluster deposition [30], filtered cathodic vacuum arc deposition [31, 32], ion beam co-sputtering [33, 34, 35], and DC [36, 37] and RF magnetron sputtering [38, 39].

The analyses performed on metal-containing amorphous carbon films, of course, depend on the field of application that the researchers are aiming at. A number of the above groups investigate the influence of the metal on the tribological properties of the films [31, 32, 38, while others focus on the behaviour of the metal within the films, i.e. cluster formation and the corresponding cluster properties in dependence 


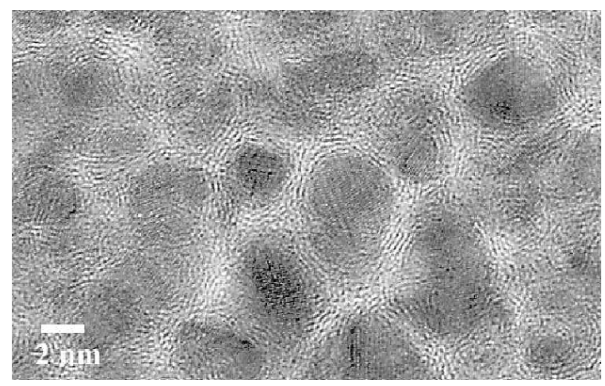

Figure 1.2: High resolution transmission electron micrograph of an a-C:Cu film with 55 at.\% copper. Layers of carbon hexagons arranged parallel to the cluster surfaces can be distinguished (from [40]).

on the deposition parameters [29, 33, 34, 36, 37].

Generally, the former find a decrease in the "diamond-like properties", such as $\mathrm{sp}^{3}$ bonding content, Vickers hardness, and wear resistance with increasing metal content. Accordingly, the stress reduction of the film is successful.

The results of the latter depend on the deposition technique, partly even on the specific setup. The pulsed laser ablated films of Benchikh and co-workers contain rather large $\mathrm{Ni}$ clusters with diameters of about $100 \mathrm{~nm}$ [29]. The $\mathrm{Ni}$ content is assumed to be close to 50 at.\%. They find evidence for the existence of Ni-C bonds; however, no quantitative information is given.

Babonneau, Cabioc'h and co-workers examined the formation of graphene cages around silver, copper, and iron nanoclusters in co-sputtered a-C:Me films [33, 40, 41, 42. Generally, the reported cluster sizes are much smaller than those in the laser-ablated films by Benchikh and co-workers, ranging from 2 to $12 \mathrm{~nm}$. For the silver-containing films, cage growth occurs at temperatures higher than $773 \mathrm{~K}$, or higher than $573 \mathrm{~K}$ if ion assistance was implemented. The particle diameters vary from around $2 \mathrm{~nm}$ for the non-assisted films to 2-12 nm with broad size distributions for the ion beam assisted films. The a-C:Cu films show a graphene cage formation without ion beam assistance even at room temperature (see Figure 1.2). This is attributed to a catalytic effect of copper that leads to the graphitization of the surrounding amorphous carbon matrix. The copper clusters in the matrix are elongated along the growth direction of the film. This is explained by a nucleation and growth process utilizing surface diffusion and demixing of carbon and copper and a resulting copper island growth. The ongoing film growth then leads to a columnar growth. The cluster diameters parallel and perpendicular to the growth direction are in the range of 5 and $7 \mathrm{~nm}$, respectively. Similar results are presentend for a-C:Fe films. In the later publication by Babonneau and co-workers, a comparison to $\mathrm{Fe}^{+}$ion implantation and post-deposition irradiation as well as co-irradiation with inert gas ions is drawn. While the non-irradiated as well as the $\mathrm{He}^{+}$ion irradiated sputter-deposited 
films show a structure of elongated iron-rich clusters, the clusters are spherical for the co- and post-deposition $\mathrm{Ar}^{+}$ion irradiated films. This is attributed to a stress reduction and the rearrangement processes during so-called thermal spikes caused by the ion impacts. Furthermore, the $\mathrm{Fe}^{+}$ion implanted films also exhibit spherical clusters. Babonneau and co-workers argue that in this case, the clusters form within the matrix, and the process is therefore not driven by surface diffusion.

Schiffmann and co-workers investigated the particle size and distance dependence on the metal concentration of gold, platinum, tungsten, and iron in a-C:H films grown by reactive gas sputtering of metal targets [34]. The various characterization techniques implemented (Transmission Electron Microscopy (TEM), Scanning Tunnelling Microscopy (STM), Small Angle X-Ray Scattering (SAXS)) show that the particle radii as well as their centre distances increase with increasing metal content. The particle diameters range from 1 to about $5 \mathrm{~nm}$. For the highest amount of incorporated metal, the particle distance is about twice the particle radius, corresponding to a closed package of metal clusters in the films. The fact that films containing carbide-forming metals show smaller particle sizes is attributed to a smaller mobility of deposited carbide molecules.

Rather extensive studies on amorphous carbon and amorphous hydrogenated carbon films containing either copper, iron, or cobalt were published by IvanovOmskiǔ, Yastrebov, and co-workers [36, 43, 44, 45, 46]. They used a planar DC magnetron setup for the deposition of a-C:H(Cu) films. The deposition setup was operated with an $\mathrm{Ar} / \mathrm{H}_{2}$ gas mixture. Different areas of a graphite target were covered by copper platelets yielding various copper concentrations. In their early publications, they present data showing a constant mean copper particle diameter of about $3 \mathrm{~nm}$ for copper concentrations up to 14 at.\%. The size distribution of a sample can be fitted by a Gaussian function. At the smallest copper content of 4 at.\% they derive a smooth sphere structure from their SAXS data, while at larger copper contents the cluster surface is found to be rather rough. A model presented by Kulikov and co-workers assumes the following: the incoming carbon and copper atoms arrive at the film surface with low energies and therefore do not penetrate the surface. The copper clusters are formed due to surface diffusion, as the diffusivity of copper within amorphous carbon is negligible. Therefore, the cluster formation process takes place only during the growth of one or two monolayers [43]. The cluster size distribution function derived from this model represents the experimental data fairly well. However, the constant cluster diameter at low concentrations presented 
in Reference [36] is not discussed and not easily understandable from the model. Furthermore, the assumption that the incoming atoms do not penetrate the surface seems rather questionable, because the magnetron power of $350-450 \mathrm{~W}$ is rather high and the deposition pressure of 1-10 mTorr is comparatively moderate.

A rather cursory study on cobalt-containing amorphous carbon films also reveals a cluster formation with a cluster diameter of approximately $7 \mathrm{~nm}$ for a film with a cobalt concentration of 18 at.\% [46]. Upon annealing at $800^{\circ} \mathrm{C}$ in an argon atmosphere, the cobalt concentration of the film is increased to 80 at.\% due to evaporation of the a-C:H host. The cluster size distribution is then considerably broader and can be fitted by two Gaussians centered at 12 and $26 \mathrm{~nm}$. No interpretation of this effect is given.

In their studies on iron-containing amorphous carbon films, Yastrebov and coworkers restricted themselves to a closer analysis of the matrix structure as a function of the metal content [47, 39]. In this case, films were grown by RF magnetron sputtering. In the first publication, they derive from Raman spectra in the range of $1000-1800 \mathrm{~cm}^{-1}$ that the matrix structure remains unchanged by the presence of iron up to a concentration of 25 at.\%. The latter study analyzes Raman spectra in the range of $200-1000 \mathrm{~cm}^{-1}$. From the features found in these spectra, the formation of short carbon nanotubes promoted by the presence of iron is suggested.

At the National Cheng Kung University of Tainan, Taiwan, various researchers work on the deposition and analysis of metal-containing amorphous carbon films. Chen and Hong grew a-C:Cu films in a pulsed magnetron with $\mathrm{RF}$ biased substrates [48]. They found clusters with diameters in the range of $15-30 \mathrm{~nm}$ embedded in an amorphous carbon matrix. Again, the main interest is the improvement of the film ductility, i.e. the reduction of stress while maintaining high hardness. The incorporation of about 11 at.\% copper at a magnetron pulse power of $280 \mathrm{~W}$ resulted in a stress reduction by a factor of 4 and an improved work load of $80 \mathrm{~N}$ as compared to $66 \mathrm{~N}$ while maintaining a reasonable film hardness of $16 \mathrm{GPa}$ as compared to $22 \mathrm{GPa}$ of a metal-free amorphous carbon reference sample. Lee and co-workers used a DC magnetron setup for the growth of magnetic $\mathrm{Fe}_{3} \mathrm{C}$ and $\mathrm{FePt}$ particles in an amorphous carbon matrix by non-reactive sputtering of graphite targets onto which pellets of the respective metals were attached [37, 49]. The as-deposited a-C:Fe films consist of clusters with sizes below $5 \mathrm{~nm}$. Selected area diffraction patterns indicate the presence of crystalline $\mathrm{Fe}_{3} \mathrm{C}$ as well as iron. Upon annealing at temperatures up to $550^{\circ} \mathrm{C}$, the iron carbide signals become more pronounced and 
(a)

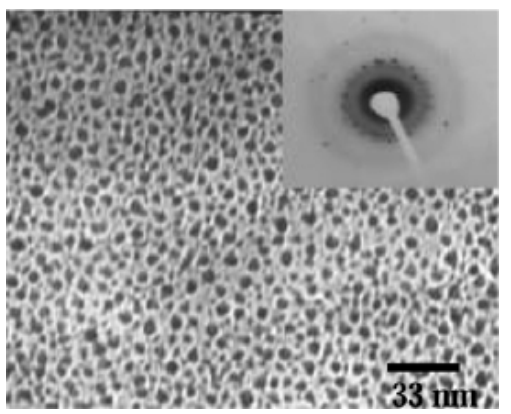

(b)

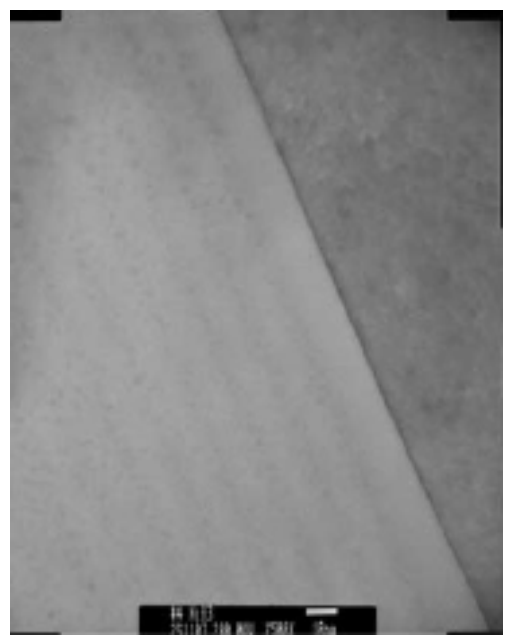

Figure 1.3: TEM micrographs of magnetron-sputtered a-C:Ni films. (a) In plane view transmission electron microscopy, nickel clusters appear to be uniformly distributed throughout an amorphous carbon matrix. (b) A cross-sectional view reveals the multilayer structure of an a-C:Ni film (from [51] and [1]).

the cluster size rises up to a maximum value of $18 \mathrm{~nm}$. At higher temperatures, the $\mathrm{Fe}_{3} \mathrm{C}$ starts to decompose. In contrast to this, the a-C:FePt films are rather insensitive to thermal treatment. All films are composed of face centered tetragonal FePt clusters of $\sim 3 \mathrm{~nm}$ size.

Ting and co-workers employed RF as well as DC magnetron setups for the deposition of amorphous carbon films containing platinum, nickel, or copper. They used an $\mathrm{Ar} / \mathrm{CH}_{4}$ gas mixture and sputtered from pure metal targets. The RF magnetrongrown a-C:Pt films show platinum clusters with cluster sizes of $1-5 \mathrm{~nm}$, the size increasing with increasing $\mathrm{Ar} / \mathrm{CH}_{4}$ ratio, which corresponds to a higher platinum concentration [38, 50]. The deposition of a-C:Pt and a-C:Cu films by $\mathrm{DC}$ magnetron sputtering results in cluster sizes below $5 \mathrm{~nm}$, while a-C:Ni films obtain clusters of 5-15 nm, increasing with the nickel concentration. From plane view TEM, Wu and Ting concluded that the clusters of all films are spherical and distributed homogeneously throughout the amorphous carbon matrix [51]. However, cross-sectional TEM analysis and depth resolved Auger electron spectroscopy revealed that the films really consist of alternating layers of densely distributed metal clusters and almost metal-free amorphous carbon layers [1]. The respective micrographs are shown in Figure 1.3. Their findings, along with their multilayer formation model, are discussed in more detail in Chapter 6 .

Recently, Corbella and co-workers published a study on the spontaneous forma- 
tion of carbon/titanium multilayers in a pulsed DC magnetron sputtering process with RF biased substrates [2]. They assumed a spinodal decomposition process that leads to a surface-directed decomposition of the growing film. The energy of the incoming species from the $\mathrm{Ar} / \mathrm{CH}_{4}$ plasma is believed to strongly enhance diffusion in a region near the surface. The formation of alternating layers is caused by the affinity of one of the components to the substrate. An important feature of their films is that the multilayers become blurred towards the film surface and the morphology passes into a homogeneous distribution of clusters. In an earlier study, the growth of carbon-metal multilayers by alternating magnetron deposition was reported [52]. In further investigations, the composition and morphology of metal-containing amorphous carbon was studied, comparing the differences of the four carbide-forming metals tungsten, molybdenum, niobium and titanium [53, 54]. The experimental conditions were the same as in the case of the self-organized multilayer films. The multilayer structure had not been revealed by TEM studies at the time of the earlier publications as only plane view images were recorded. The results are therefore summarized along with a more detailed description of the experimental findings on the multilayer films and the model approach in Chapter 6.

\subsection{Examples of Self-Organization}

The development of techniques of industrial interest for the fabrication and optimization of nanostructured materials is based on a thorough understanding of the nucleation and growth processes. In this context, self-organization mechanisms are especially appealing for both technological and scientific reasons. Of course, the term self-organization is somewhat vague, since there is always a physical force driving the process. In this work, the definition of the "Lexikon der Physik" [55] will provide the basis for discussion: Self-organization refers to a spontaneous evolution of stable, ordered structures in dissipative systems.

Self-organization occurs in any order of magnitude, ranging from star clusters to just a few atoms [56]. It is of considerable value for the generation of lowdimensional semiconductor structures, as lithography and etching-based fabrication are rather complicated processes. A number of examples are given by Moriarty [57]: semiconductor nanoclusters with narrow size-distributions grow self-assembled on semiconductor substrates due to a lattice mismatch of the cluster and substrate materials. Also, lateral positioning of clusters can be controlled by using a suitably 
prepared substrate.

An organization into columnar structures has been observed in epitaxial LSMO (Lanthanum-Strontium-Manganate) films grown by pulsed laser deposition onto $\mathrm{LaAlO}_{3}$ (Lanthanum-Aluminum-Oxide) [58]. In the initial stage, a few monolayers grow coherently onto the substrate, followed by a misfit dislocation formation at a critical thickness and a proceeding island-like rough surface structure formation. The islands are believed to be unstressed regions that act as nucleation sites for the columns. The valleys are filled with material of much lower density and show an amorphous structure. Babonneau and co-workers [59] also found a columnar structure for Fe-BN films grown by ion beam co-sputtering of a BN disk with an attached iron platelet if an assistance ion beam is applied. The columnar structure arises for both reactive and inert gas ion beam assistance. However, a short range ordering is only observed when the assistance ion beam is inert. The effect is attributed to advanced surface diffusion.

A technique of growing films with a multilayer structure that is often associated with the term self-assembly is the Langmuir-Blodgett growth. Langmuir and Blodgett developed a technique to spread a monomolecular layer of fatty acids onto an air/water interface. The molecules self-assemble in such a way that the carboxyl group is in contact with the water and the hydrocarbon chains are oriented perpendicular to the surface. The film is transferred to a substrate by dipping. In the first dip, the carboxyl group attaches to the substrate. When the substrate is lowered into the water again, the second layer is attached, the third when it is raised again and so on. This dipping process results in a multilayer film with an opposite molecular orientation of the successive layers [60]. This technique has gained renewed attention starting in the 1980s in connection with the advancement of the field of molecular electronics. A review of the recent development is given in Reference [61].

Another chemical process resulting in multilayer structured films is the layerby-layer self-assembly. This process relies on the self-assembled adsorption from alternately polyanionic or polycathionic aqueous solutions onto a charged substrate 62]. The substrate is dipped alternately into each solution. However, this technique is not strictly a self-organization process for the purpose of the above definition.

Fukunaga and co-workers report on the self-assembly of lamellar ABC triblock co-polymer films into multilayer systems during solvent-vapour treatment [63]. The as-prepared dip-coated films are of a sponge-like structure. When exposed to tetrahydrofuran at room temperature, the films start to organise into layers of the differ- 
ent polymers starting from the surface.

A mechanism often leading to some form of self-organized structure is spinodal decomposition. In the case of supercooling, an alloy is unstable against concentration fluctuations when the second derivative of the molar free energy with respect to the concentration of one component is negative. This results in a negative interdiffusion coefficient, which may, for instance, lead to the formation of periodic structures known as Liesegang patterns [64, 65]. Depending on the initial geometry, rings or layers are formed in a diffusion process of one electrolyte into a material containing another electrolyte. At certain depths, the concentration of both electrolytes rises above a threshold level and precipitation occurs. The position of the precipitation front is $\propto \sqrt{t}$ ( $t$ is the time), which is a direct consequence of the diffusive nature of the process. The evolution of nearly periodic concentration variations of $\mathrm{Cd}$ over-stoichiometric ion implanted CdSe nanocrystals reported by Grosshans and co-workers is very closely related to the Liesegang pattern formation [66].

The above-mentioned self-organization processes basically result from one of the following: either, the conditions given by the surroundings, the chemistry of the system, or equilibrium thermodynamics. This is important to note for the understanding of the structure formation processes described in this thesis. It will be discussed further below that all of the processes described in this section can be excluded from being responsible for the formation of the layered structures of this work. 


\section{Chapter 2}

\section{The Base Materials}

\subsection{Carbon}

Carbon is a very versatile element. Most widely known are the two crystalline phases graphite and diamond. Graphite is composed of layers of hexagons; each atom is $\mathrm{sp}^{2}$-hybridized and is bonded to three neighbours. The layers interact by weak Vander-Waals forces and may slip with respect to each other. Therefore, graphite is a very soft material and frequently used as a lubricant. In contrast to this, diamond is to date the hardest known material. The carbon atoms in a diamond lattice are $\mathrm{sp}^{3}$-hybridized, which leads to a tetragonal atomic structure with very strong bonds. The graphite and diamond lattices are schematically shown in Figure 2.1.
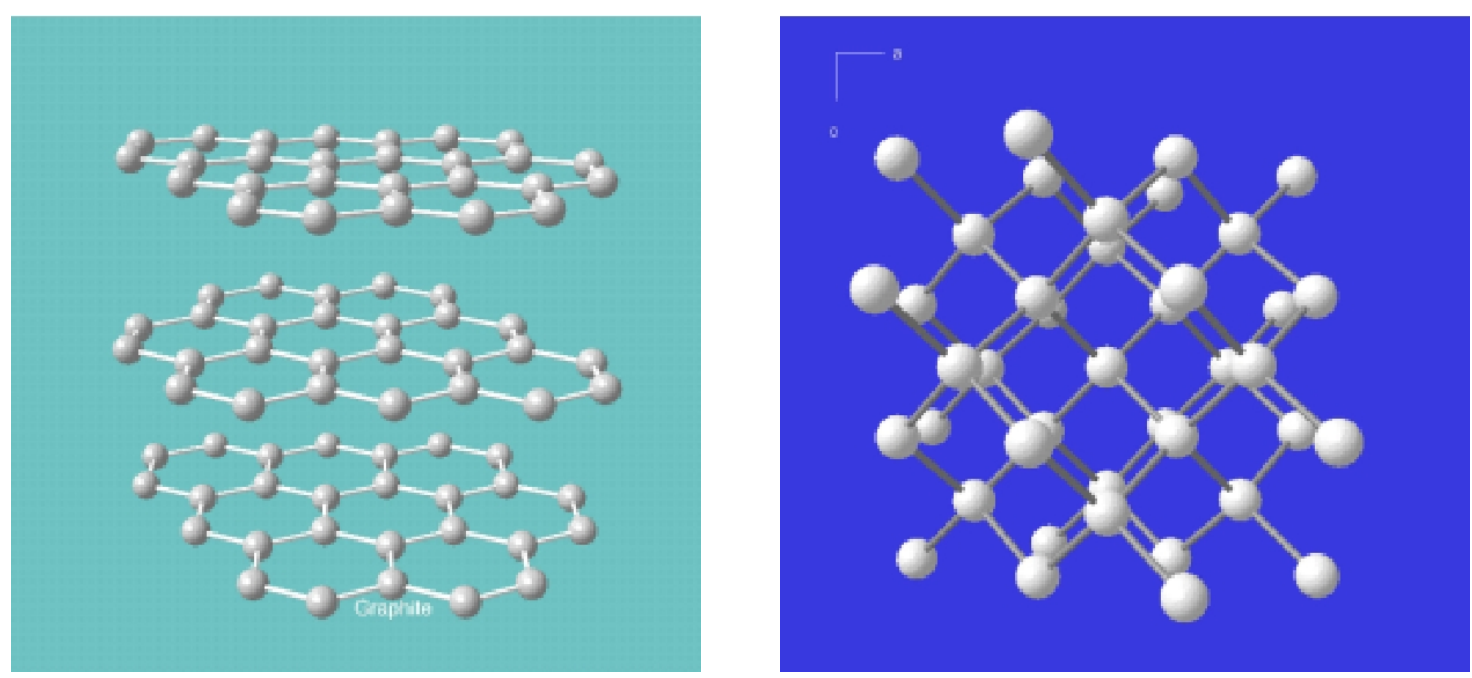

Figure 2.1: Schematic of the atomic structures of graphite (left) and diamond (right) (from [67]). 


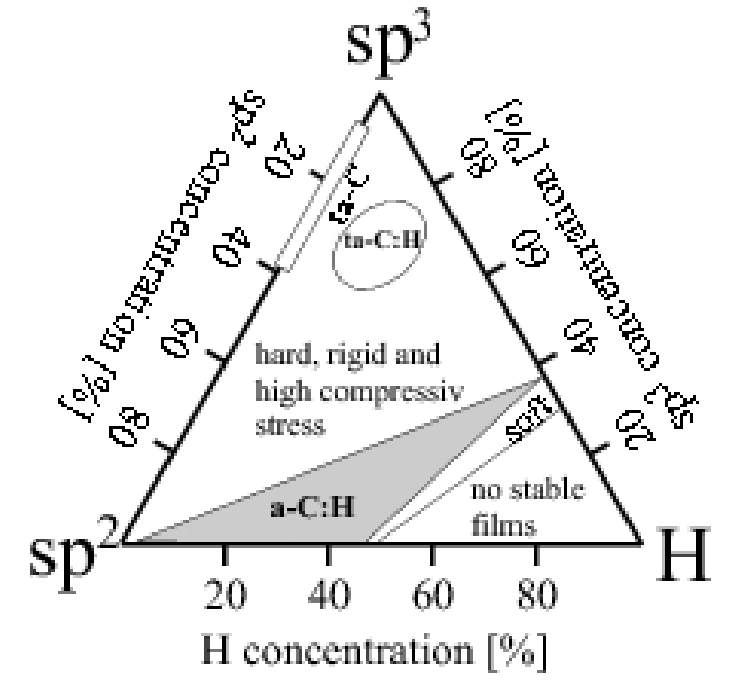

Figure 2.2: Ternary phase diagram of $\mathrm{sp}^{2}$ and $\mathrm{sp}^{3}$-bonded amorphous carbon and hydrogen (from [70] and references therein).

The crystal structure of graphite is hexagonal close packed with the lattice constants $a=2.4612 \AA$ and $c=6.7079 \AA$ and the space group is $\mathrm{D}_{6 \mathrm{~h}}{ }^{4}$. The diamond crystal structure is face centred cubic with two base atoms. The second atom is shifted with respect to the first in the direction of the space diagonal of the unit cell by one quarter of the length of the diagonal. The lattice constant is $a=3.5670 \AA$. The differences between graphite and diamond stretch far beyond their hardness values: graphite is a black metallic conductor, while diamond is a transparent wide band gap semiconductor $\left(E_{g}=5.45 \mathrm{eV}\right)$ with excellent thermal conductivity and a high index of refraction.

Additionally, there is the large class of amorphous carbon structures ranging from fully $\mathrm{sp}^{2}$-bonded material to films with over $85 \% \mathrm{sp}^{3}$-hybridized carbon atoms. Depending on the deposition method, the material may also contain significant amounts of hydrogen. Figure 2.2 shows the ternary phase diagram covering the amorphous carbon and amorphous hydrogenated carbon materials. The hardness values of the hydrogen-containing phases are usually of the order of 20-30 GPa, but the material can still posses a number of high quality properties, such as a large band gap, high index of refraction, chemical inertness, and a very low friction coefficient [70]. With increasing $\mathrm{sp}^{3}$-bonding content, towards the tip of the phase diagram in Figure 2.2 , the material becomes harder and its properties become more diamond-like.

In the literature, the term diamond-like carbon (DLC) has been stretched somewhat beyond its definition for amorphous carbon containing virtually any amount of $\mathrm{sp}^{3}$-bonded atoms. However, the material's properties approach the extreme values of diamond only when the film is free of hydrogen and the $\mathrm{sp}^{3}$-bonding content 
Figure 2.3: Calculated atomic structure of amorphous carbon. Blue atoms are $\mathrm{sp}^{3}$-bonded, green atoms $\mathrm{sp}^{2}$-bonded (from [68]).

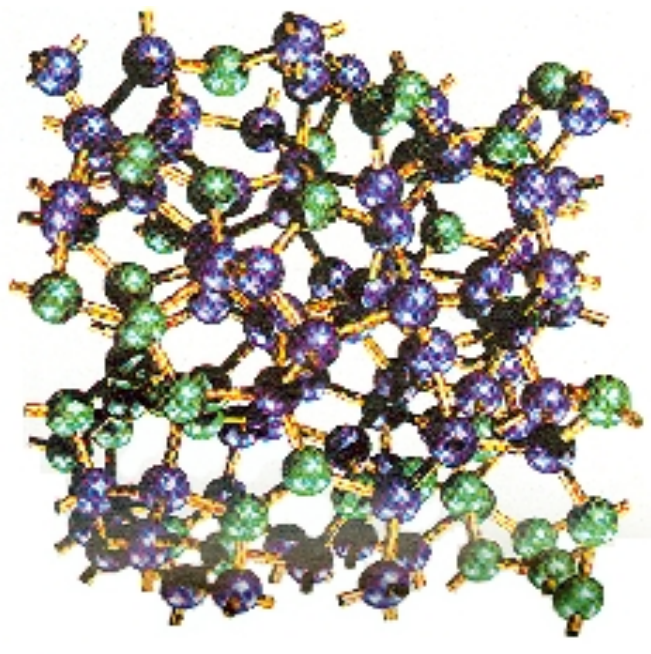

is $\geq 80 \%$; such a material is then called tetrahedrally bonded amorphous carbon (ta-C). Figure 2.3 shows an amorphous carbon structure with a certain fraction of $\mathrm{sp}^{3}$-bonded atoms which was calculated using molecular dynamics simulations.

Ta-C has been the subject of research in our group for several years (e.g. References $[69,70,71,72])$. The excellent properties of ta-C thin films deposited using the mass selective ion beam setup ADONIS are given in Table 2.1 in comparison with the physical properties of diamond. Compared to chemical vapour-deposited diamond films, ta-C films show a very low surface roughness $(<1 \mathrm{~nm})$ but bear a rather high internal compressive stress of $4-15 \mathrm{GPa}$ [70]. This high internal stress limits the maximum film thickness, because the films peel off when a critical thickness is reached. Some effort is made to decrease the internal stress of amorphous carbon films, either by thermal treatment (e.g. References [73] and [74]) or by incorporation

Table 2.1: Properties of ta-C films deposited using the mass selective ion beam deposition setup ADONIS compared with the properties of diamond (from [70]).

\begin{tabular}{lrr}
\hline \hline & ta-C & Diamond \\
\hline Hardness [GPa] & $\sim 40-80$ & 100 \\
Density [g/cm $\left.{ }^{3}\right]$ & $\sim 3$ & 3.51 \\
$\mathrm{sp}^{3}$ Bonding Fraction & $>80 \%$ & $100 \%$ \\
Spec. Resistivity $[\Omega \mathrm{cm}]$ & $\sim 10^{10}$ & $>10^{16}$ \\
Band Gap [eV] & $\sim 2-3$ & 5.45 \\
Index of Refraction & $\sim 2.5$ & 2.4 \\
Transparency & $\mathrm{UV}-\mathrm{IR}$ & $\mathrm{UV}-\mathrm{IR}$ \\
Thermal Conductivity $[\mathrm{W} /(\mathrm{cmK})]$ & $1-7$ & 20 \\
\hline \hline
\end{tabular}


of foreign atoms (e.g. Reference [75]). As mentioned above, incorporating metals is another attempt to improve the tribological properties of a-C. All of the attempts mentioned before eventually lead to a reduction of the $\mathrm{sp}^{3}$-bonding content, mostly accompanied by a degradation of the diamond-like properties. In this work, one aim is to maintain the high standard of the properties of the embedding matrix that were achieved for pure ta-C films, even though the metal is introduced.

\subsection{Metals of the Copper Group and Iron}

The deposition techniques employed in this study result in growth processes far from thermodynamic equilibrium. Nevertheless, it is useful to have a look at the thermodynamic properties of the bulk of the metals and their chemical behaviour in the presence of carbon.
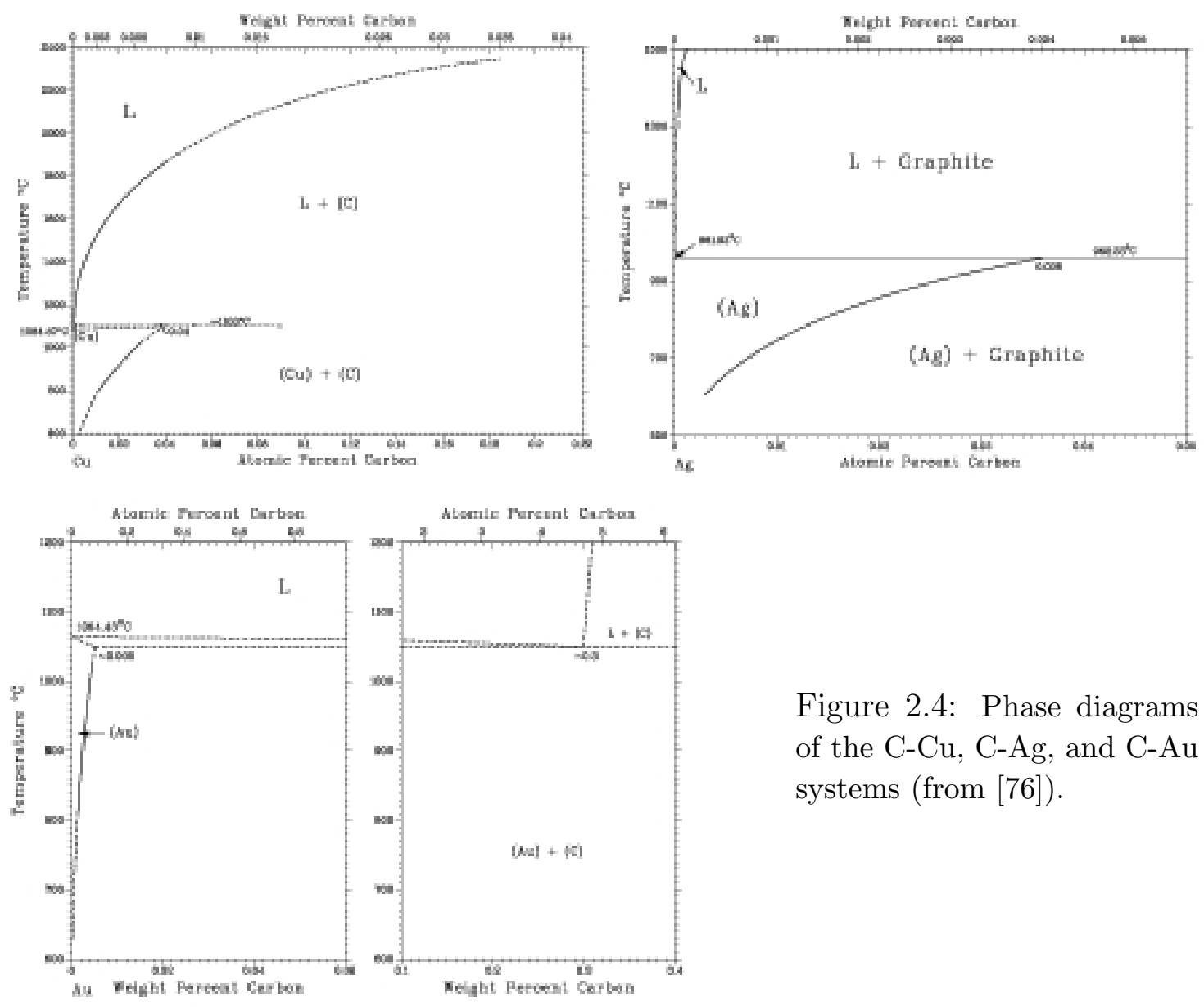

Figure 2.4: Phase diagrams of the $\mathrm{C}-\mathrm{Cu}, \mathrm{C}-\mathrm{Ag}$, and $\mathrm{C}-\mathrm{Au}$ systems (from [76]). 
Figure 2.5: Phase diagram of the C-Fe system (from [77]).

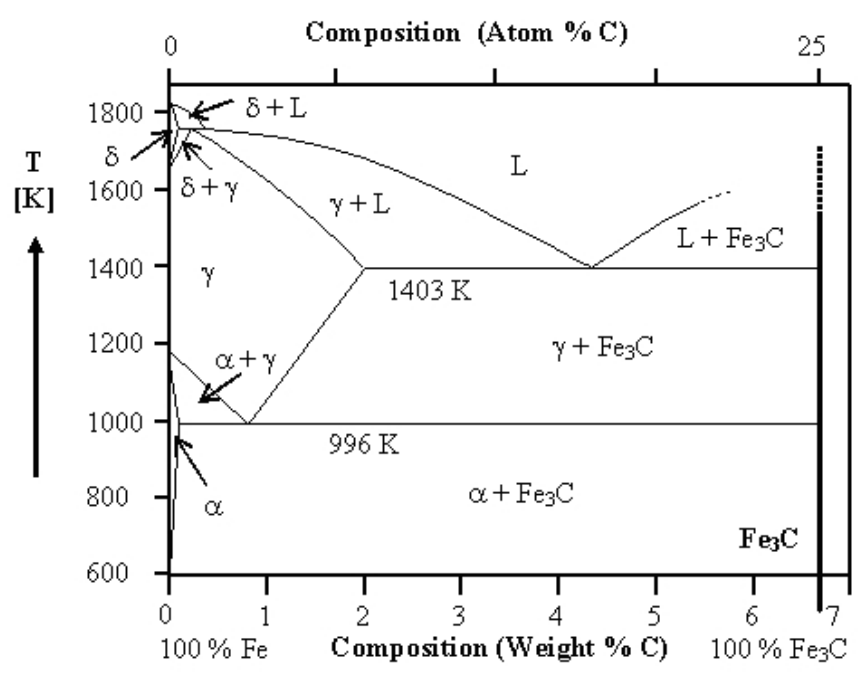

The three metals of the copper group all have a face centred cubic crystallographic structure. They are malleable and ductile, and good electrical and thermal conductors. Non of them are carbide-forming and the mutual solubilities of carbon and each metal are negligibly small, as it can be seen in the phase diagrams in Figure $2.4[78,79,80,81]$.

The iron-carbon system is somewhat more complex. The respective equilibrium phase diagram is displayed in Figure 2.5. At very small carbon concentrations, the body centred cubic $\alpha$-iron (ferrite) and, at higher temperatures, $\delta$-iron phases can be found. Furthermore, the face centred cubic $\gamma$-iron (austentite) may contain up to 8.3 at.\% carbon at interstitial sites. At higher carbon concentrations and lower temperatures, $\alpha$-iron and the stable orthorhombic $\theta-\mathrm{Fe}_{3} \mathrm{C}$ (cementite) phases are present; at elevated temperatures, $\gamma$-iron and $\theta-\mathrm{Fe}_{3} \mathrm{C}$ are found.

Additionally, there is a group of metastable phases, which can only be obtained by non-equilibrium processes: the body centred tetragonal martensite, in which carbon is incorporated at interstitial sites, and the monoclinic $\chi-\mathrm{Fe}_{5} \mathrm{C}_{2}$ (Hagg-carbide), hexagonal $\epsilon$-Fe $\mathrm{C}_{2-x}$ ( $\epsilon$-carbide), or orthorhombic $\eta$-Fe $\mathrm{F}_{2} \mathrm{C}$ ( $\eta$-carbide). All of the phases can be synthesized using ion implantation of carbon into iron films and consequent thermal treatment [86]. It is important to note that the highest carbon concentration of all these carbides is 33 at.\% in the case of $\eta$-carbide. 


\subsection{Structural Properties of Small Metal Clusters}

When only a small number of atoms of a specific element agglomerate to form a cluster, the structural properties of this cluster are likely to differ greatly from the bulk properties. In an ion beam growth process, where a rather large amount of energy is transferred to the growing material, the stability of a small cluster may be an important factor determining the final film structure.

Two related types of models describing the electronic properties of simple metal clusters can be found in the literature: the shell models and the jellium models [87, 88, 89, 90; for reviews cf. References 91 and 92. Both model types rely on rather simple assumptions. The ionic cores are treated as a uniform positively charged background. The shell models describe the valence electrons using an effective single particle potential - e.g. a spherical square well potential for spherical clusters, a harmonic potential for spheroidal, or a distorted harmonic potential for ellipsoidal clusters. Such potentials result in spherical shell structures due to their symmetry, with the valence electrons successively filling the degenerate states. As opposed to this, the jellium model treats the valence electrons self-consistently, while the positively charged ionic background (the jellium) may be spherical, ellipsoidal or otherwise deformed. Despite their strong simplifications, both models are surprisingly successful in reproducing experimental data, such as magic numbers and
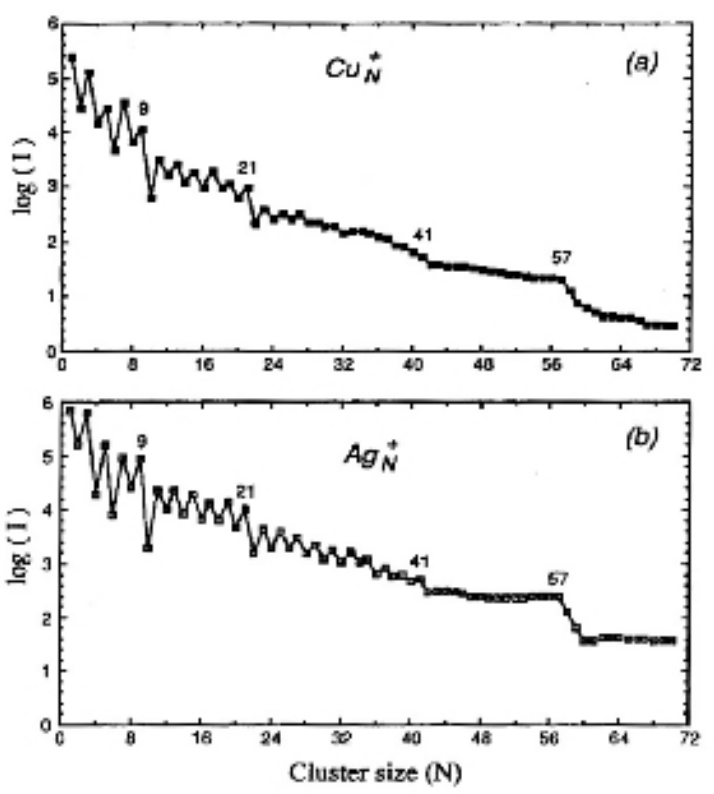

Figure 2.6: Mass abundance spectra of (a) copper and (b) silver clusters. An increased number of clusters is found for atom numbers corresponding to closed shells according to the shell model (from [91]). 

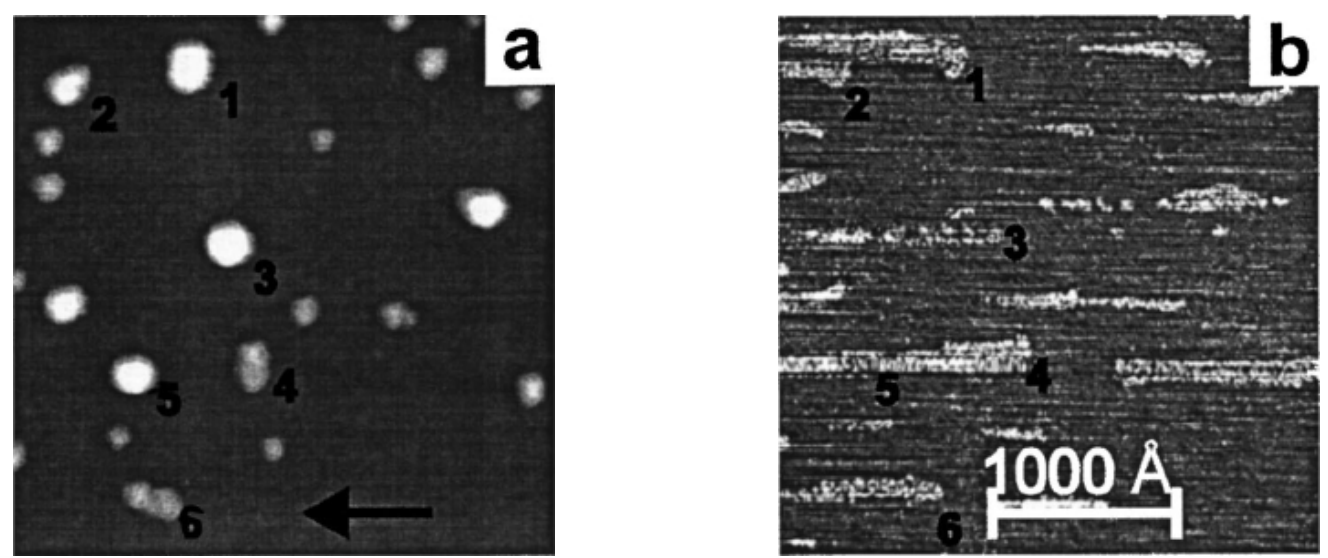

Figure 2.7: Silver clusters distributed on silicon surface (a) before and (b) after manipulation with the STM tip. The numbers are to guide the eye in identifying the original positions (from [95]).

the fine structure of the mass abundance spectra. Figure 2.6 shows the mass abundance spectra of copper and silver clusters generated by a sputter cluster ion source employing a $20 \mathrm{keV}$ krypton ion beam [91]. The spectra show pronounced steps at cluster sizes corresponding to the predictions of the shell model. The closed shells at atom numbers $N=8,20,40,56, \ldots$ are surpassed always by one atom due to the fact that the spectroscopy was performed on singly charged cluster cations. The higher abundance of clusters at electronic shell closures implies that these clusters are more stable than agglomerations of other numbers of atoms. Fragmentation preferentially produces clusters with closed shells.

Despite the astonishing success the two models described above, their neglect of the geometric structure of the ionic background is a problematic drawback. The studies by Winter and co-workers and Fournier suggest that clusters of the two metals copper and silver show indeed very different structural behaviour [93, 94]. Although both their electronic properties are well described by the jellium and the shell model, Winter and co-workers suggest that copper clusters also show an icosahedral geometrical structure, while Fournier finds silver clusters to appear as liquid droplets with large vibrational amplitudes and many different isomers for a given number of atoms. Such a structural difference is supported by further experimental evidence: in an experiment by Chey and co-workers [95], copper and silver clusters were attempted to be moved across $\operatorname{Si}(111)-(7 \times 7)$ surfaces using the tip of a scanning tunnelling microscope (STM). The copper clusters could not be moved and broke when the applied force was too strong. The attempt to move silver clusters 
with the STM tip was successful and resulted in a track of silver atoms along the path the cluster was moved - an effect described as nanopainting (see Figure 2.7). It can be concluded that the Ib-group metals behave rather differently when reduced to agglomerates of only a few atoms, and therefore differences in the structures of their nanocomposites are not surprising.

\subsection{Nucleation and Growth of Thin Metal Films on Carbon Substrates}

The transport of atoms arriving at the surface of a substrate at thermal energies mainly includes adsorption, desorption and surface diffusion. The rate of adsorption is dominated by the vapour pressure and the square root of both the molecular weight of the particles and the source temperature. For the other two processes, activation energies have to be overcome and the rates at which each of them occurs is given by a respective Arrhenius law. Another important mechanism that proceeds in the same manner is the capture of adatoms by already existing agglomerates, which is, of course, the main requirement for the growth of a film.

There are three basic growth modes: layer or Frank-van de Merve growth, island or Volmer-Weber growth, and the combined layer and island or Stranski-Krastanov growth. Figure 2.8 schematically shows their main characteristics. The mode in which a film grows is determined by the strength of the cohesion forces between the film atoms and the adhesion forces between the film atoms and the substrate atoms. Frank-van der Merve growth is the result if the adhesion forces are predominant. Therefore, a full coverage of the substrate with one monolayer of film atoms is completed before the next monolayer starts growing. On the contrary, when the cohesion forces dominate, the film atoms form small three-dimensional islands on the substrate (Volmer-Weber growth). The film closes only when the density of islands

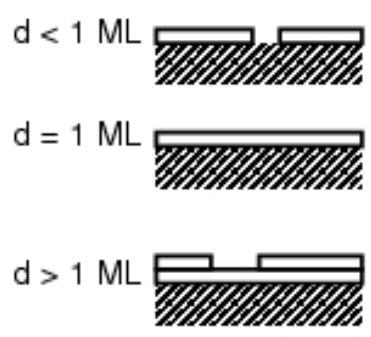

(a)

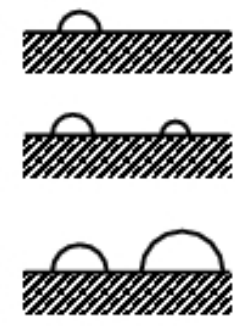

(b)

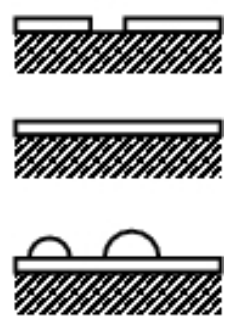

(c)
Figure 2.8: Growth modes of thin films: (a) Frank-van de Merwe (b) Volmer-Weber and (c) Stranski-Krastanov. 
Figure 2.9: An island on a substrate surface. $\gamma_{S}, \gamma_{F}$, and $\gamma_{S / F}$ are the surface tensions of the the substrate and the film and the interface tension between the substrate and the film, respectively. The direction of these tensions are given by the forces resulting from adhesion and cohe-

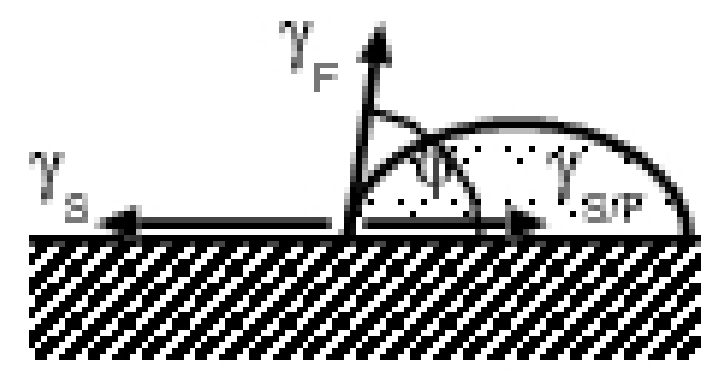
sion.

becomes large and the islands coalesce. The Stranski-Krastanov growth is an intermediate case. After the deposition of one or even several monolayers according to layer growth, the mode switches to island growth. This is predominantly generated by a lattice mismatch between the substrate and the film that cannot be maintained into the bulk.

The growth mode can be determined by evaluating the involved surface and interface tensions, which are given by a force per unit length. Regarding the case depicted in Figure 2.9, one finds that the equilibrium of forces for an island on a substrate is accomplished for $\gamma_{S}=\gamma_{S / F}+\gamma_{F} \cos \varphi$. This results in the following selection rules for the growth modes:

- Frank-van de Merwe growth: $\gamma_{S} \geq \gamma_{S / F}+\gamma_{F}, \quad \varphi=0$

- Volmer-Weber growth: $\quad \gamma_{S}<\gamma_{S / F}+\gamma_{F}, \quad \varphi>0$

If the substrate and film materials exhibit a lattice matching, and the substrate is of good crystalline quality, epitaxial layer growth is favoured. Otherwise, island growth predominates as in the case of metals on alkali halides, mica, or graphite [96]. The presence of defects then profoundly affects the growth of the islands, as the binding energy of adatoms to defects is much higher than that to the plain surface. More detailed information on the growth of thin films by evaporation can be found in the book by Lüth [97] and the review article by Reichelt [96].

Ion beam sputtered films of copper, gold, platinum, and nickel were grown on evaporated amorphous carbon films at room temperature or substrate temperatures up to $300^{\circ} \mathrm{C}$ by $\mathrm{Xu}$ and co-workers [98, 99, 100]. They studied the particle size distribution and deposition rate as a function of the sputtering parameters as well as the fractional substrate coverage as a function of the equivalent thickness of the film. From their observations they conclude an island growth for all the examined 
Au
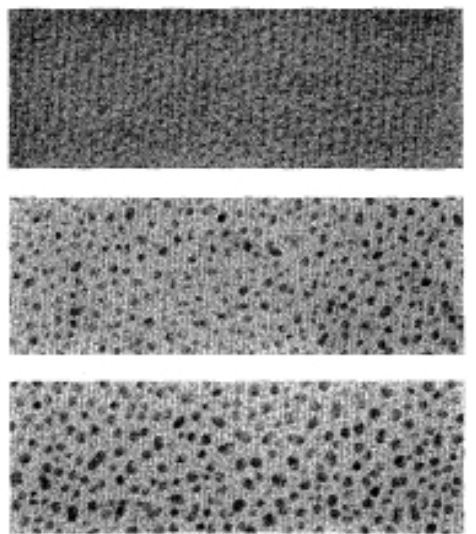

20
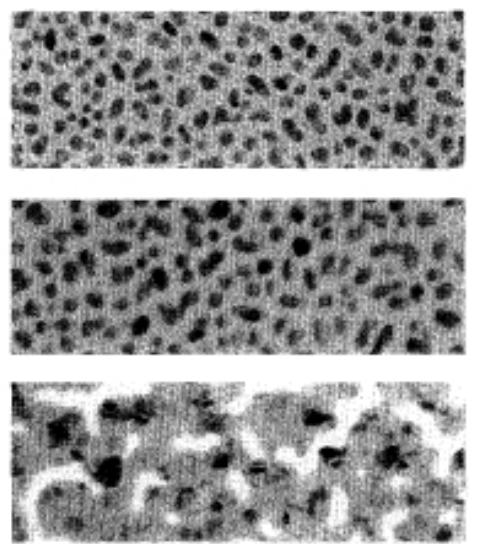

70

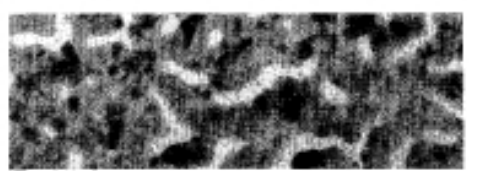

90

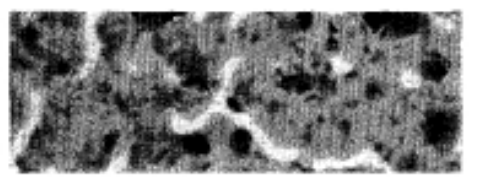

100

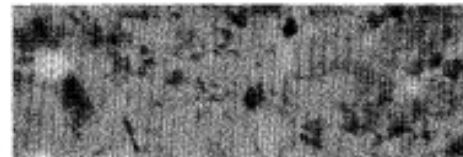

$\mathrm{Cu}$

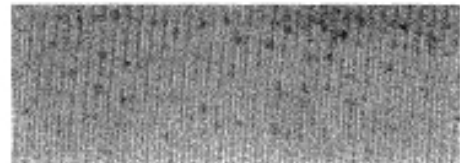

10

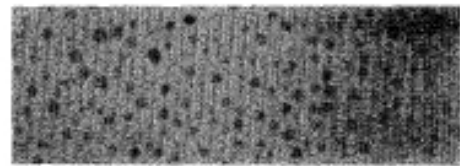

15

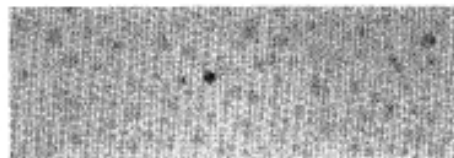

20
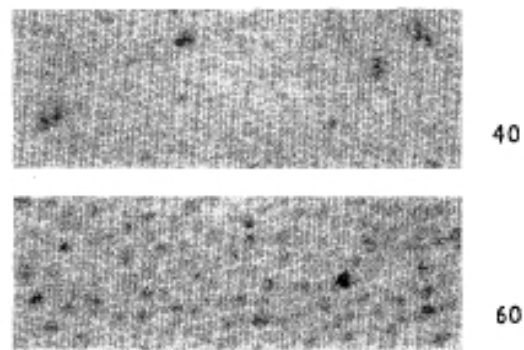

60

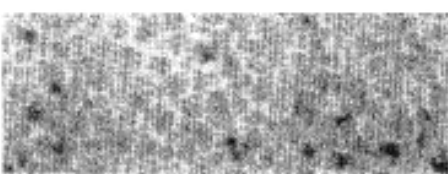

100
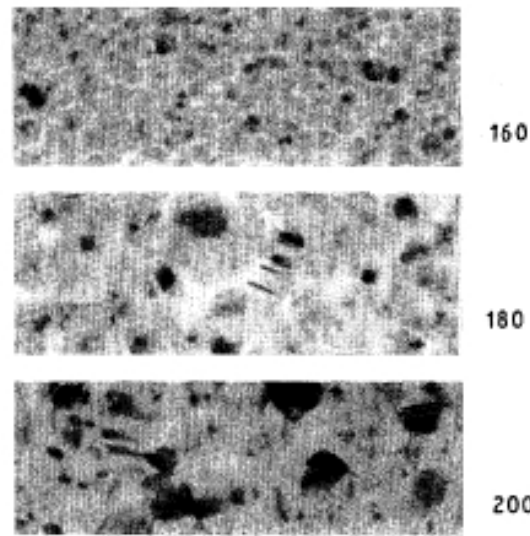

200

$50 \mathrm{~nm}$

$50 \mathrm{~nm}$

Figure 2.10: TEM micrographs of gold and copper islands grown by ion sputtering onto amorphous carbon substrates at room temperature. The number next to each micrograph denotes the equivalent thickness of the respective film in $\AA$ (from [98]). 
metals (copper, gold, platinum, and nickel). Platinum and nickel coalesce already at small equivalent thicknesses and the small islands are observed to grow mainly in two dimensions into a disc-like shape. However, gold and copper islands grow in three dimensions into hemispherical shape and coalescence starts only at equivalent thicknesses of $20 \AA$ and $40 \AA$, respectively (see Figure 2.10). A full coverage of the substrate can be observed at an equivalent thickness of $100 \AA$ in the case of gold and at $210 \AA$ for copper. It is also found that the island size in the case of gold films depends on the voltage of the sputtering source in very much the same manner as on the substrate temperature: A higher source voltage results in larger islands. The sputtering source voltage also influences the energy at which the metal atoms arrive at the substrate and therefore the mobility of the atoms on the surface is enhanced.

In more recent studies, the tendency of a preferred island growth of metals on amorphous carbon was verified by Borchers and co-workers [101, 102]. They examined the structure of ultrathin metal/light element multilayer films in view of potential application as X-ray mirrors. The growth of both nickel and chromium/amorphous carbon multilayers shows the following: while carbon wets the metal, the metal grows in islands on the amorphous carbon layers. Borchers and co-workers explain this by the surface energies of nickel, chromium and amorphous carbon: $\gamma_{\mathrm{a}-\mathrm{C}} \sim 0.5 \mathrm{~J} / \mathrm{m}^{2}, \gamma_{\mathrm{Ni}} \sim 2.3 \mathrm{~J} / \mathrm{m}^{2}, \gamma_{\mathrm{Cr}} \sim 2.0 \mathrm{~J} / \mathrm{m}^{2}$ (see References [103] and [104]). The surface tensions of the metals are larger than the surface tension of amorphous carbon. Bilamiuk and Howe also found values for the interface energies in between the values of the metal and amorphous carbon. Therefore, a carbon surface is energetically favourable.

The theoretical surface tensions for copper, silver, gold and iron are: $\gamma_{\mathrm{Cu}} \sim$ $1.9 \mathrm{~J} / \mathrm{m}^{2}, \gamma_{\mathrm{Ag}} \sim 1.3 \mathrm{~J} / \mathrm{m}^{2}, \gamma_{\mathrm{Au}} \sim 1.6 \mathrm{~J} / \mathrm{m}^{2}$, and $\gamma_{\mathrm{Fe}} \sim 2.9 \mathrm{~J} / \mathrm{m}^{2}$ [104. These values confirm island growth for gold and copper on amorphous carbon, and island formation can also be expected when silver or iron atoms emerge at the surface of amorphous carbon films. 


\section{Chapter 3}

\section{Film Growth}

\subsection{Mass Selective Ion Beam Deposition (MSIBD)}

From the summary of the status of research on metal-containing amorphous carbon in Section 1.2, it can be concluded that the atomic structure of carbon-metal composite films and their morphology on the nanoscale strongly depends on the deposition method used. Even the specific setup geometry can play a major role in determining the final film structure. In general, the specific parameters that are of great influence cannot easily be varied separately or even be precisely controlled. Therefore, it is difficult to gain a general understanding. Mass selective ion beam deposition (MSIBD) is a suitable tool to achieve a better knowledge of such films. MSIBD is a rather unique thin film growth technique. Its properties are especially valuable for the understanding of growth processes rather than technological applications. The strong advantage is that the deposition parameters can be varied independently and are quite well defined. The species to be deposited are filtered to an isotopically pure ion beam and the ion energy can be selected to meet the requirements of the experiment. The samples discussed in the present work were deposited on either of the two MSIBD setups available at the $2^{\text {nd }}$ Institute of Physics of the University of Göttingen.

\subsubsection{The MSIBD Setups Adonis and Mr. Stringer}

The two MSIBD setups are very comparable but differ in a few details. For both of them, the ions are extracted from the ion source by a voltage of up to $30 \mathrm{keV}$. The ions are filtered by a $90^{\circ}$ separation magnet and focused by an ion optical setup into the respective deposition chamber. The ion beam is scanned across the substrate 


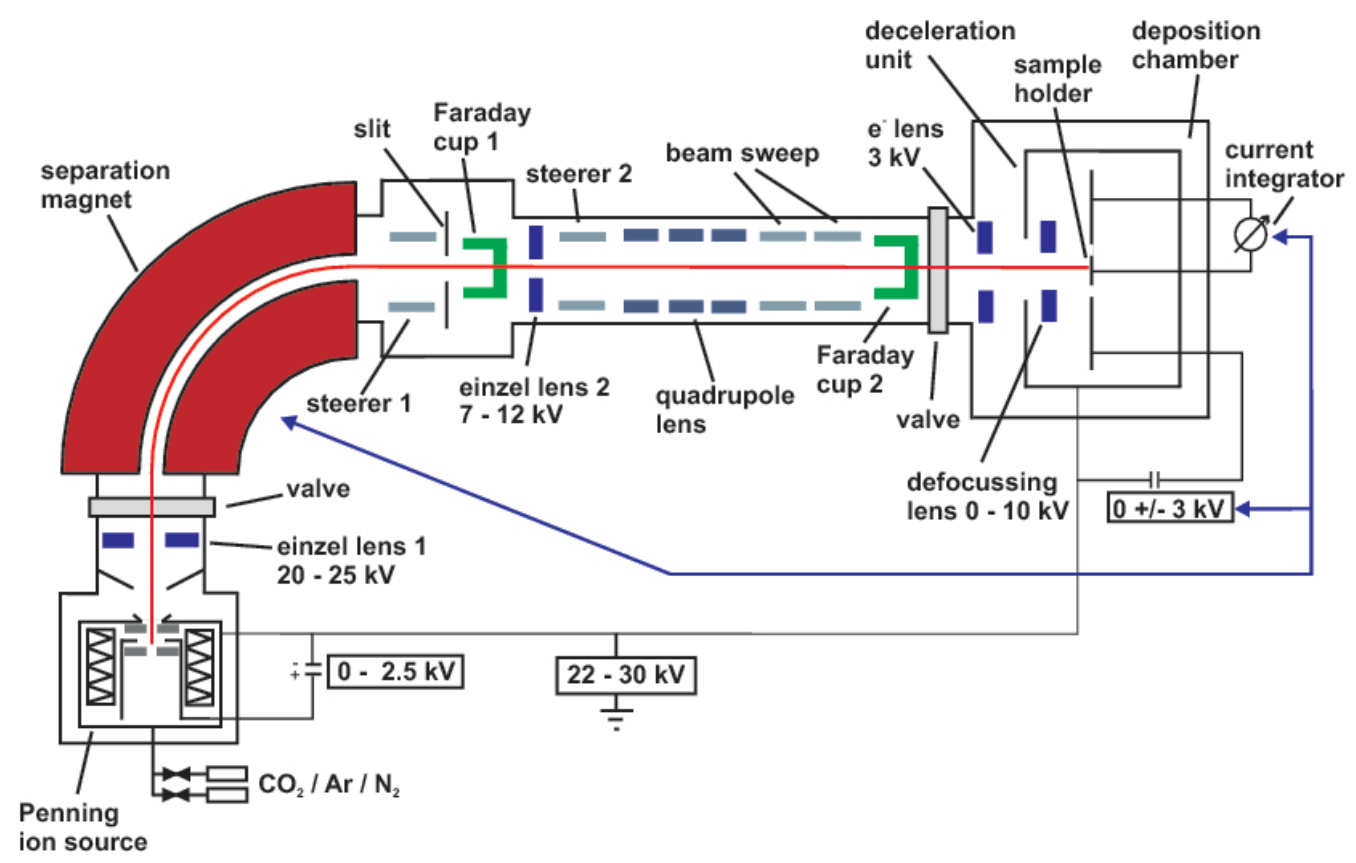

Figure 3.1: Schematic of the MSIBD setup Mr. STRINGER.

by a beam sweep in order to assure a good lateral film homogeneity. Just before impinging on the substrate, the ions are decelerated to the desired kinetic energy; for the films presented here, the ion energy values range from 40 to $200 \mathrm{eV}$. The ion current is measured on the substrate and time integrated. This way, the deposited charge is determined and can be used in order to steer the separation magnet and deceleration unit. The differences between the two set-ups lie mostly in the ion sources, deposition base pressures, and specifications of the ion optical setup.

\section{Specific Properties of Mr. StRINGeR}

Figure 3.1 schematically shows the setup of Mr. STRINGER. A set of Frankfurt type penning ion sources [105], including a pure gas source, a source operating with a sputter gas and a sputter target, and a source equipped with a heating coil for the evaporation of solids, is available for this deposition setup. The great advantage of this type of ion source is the high currents that can be produced, especially for metal ions. However, a high discharge voltage has to be applied in order to ignite the plasma. This results in a rather broad energy distribution of the extracted ions of about $60 \mathrm{eV}$. The beam line and the deposition chamber are kept at pressures $\leq 10^{-6}$ mbar. 


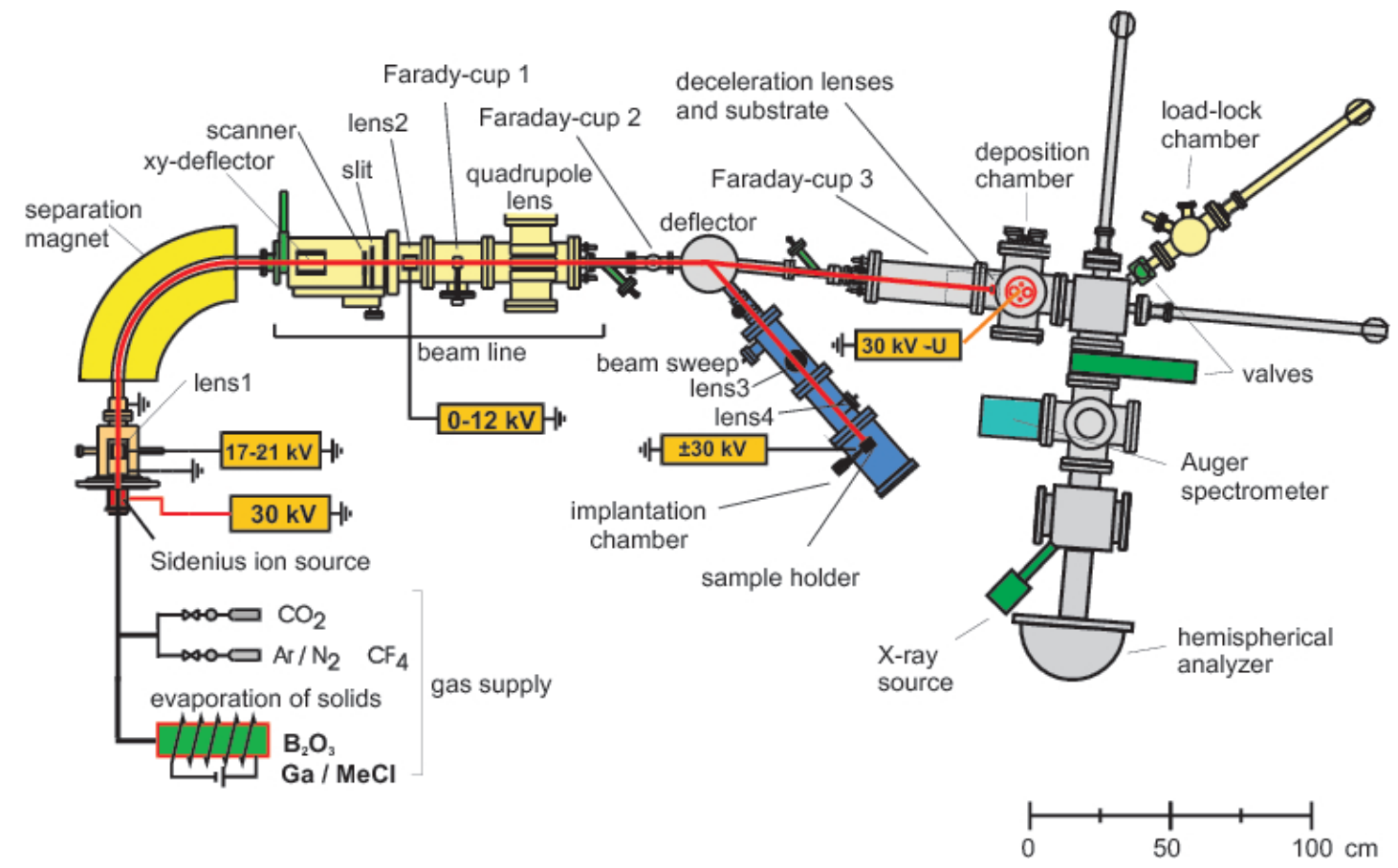

Figure 3.2: Schematic of the MSIBD setup Adonis.

\section{Specific Properties of ADONIS}

Figure 3.2 gives an overview of the MSIBD setup Adonis. Adonis is operated with Sidenius type hot filament hollow cathode ion sources [106. This type of source produces carbon ions from $\mathrm{CO}_{2}$ gas and metal ions from evaporated metals or metal chlorides. The advantage over the Frankfurt penning ion sources used at Mr. Stringer is the very narrow energy distribution of less than $10 \mathrm{eV}$.

By differential pumping, a pressure of $\approx 10^{-8}$ mbar is achieved during deposition. A $5^{\circ}$ deflection capacitor filters all recombined atoms from the ion beam just as it enters the UHV deposition chamber. By this means, the deposition process is kept very clean and very well controllable, as only the selected species are deposited.

Furthermore, ADONIS is equipped with a chamber for surface analysis by electron spectroscopy, including Auger electron spectroscopy (AES), electron energy loss spectroscopy (EELS) and X-ray as well as UV photoelectron spectroscopy (XPS and UPS). This chamber is directly linked to the deposition chamber and the sample can be transferred under UHV conditions. 


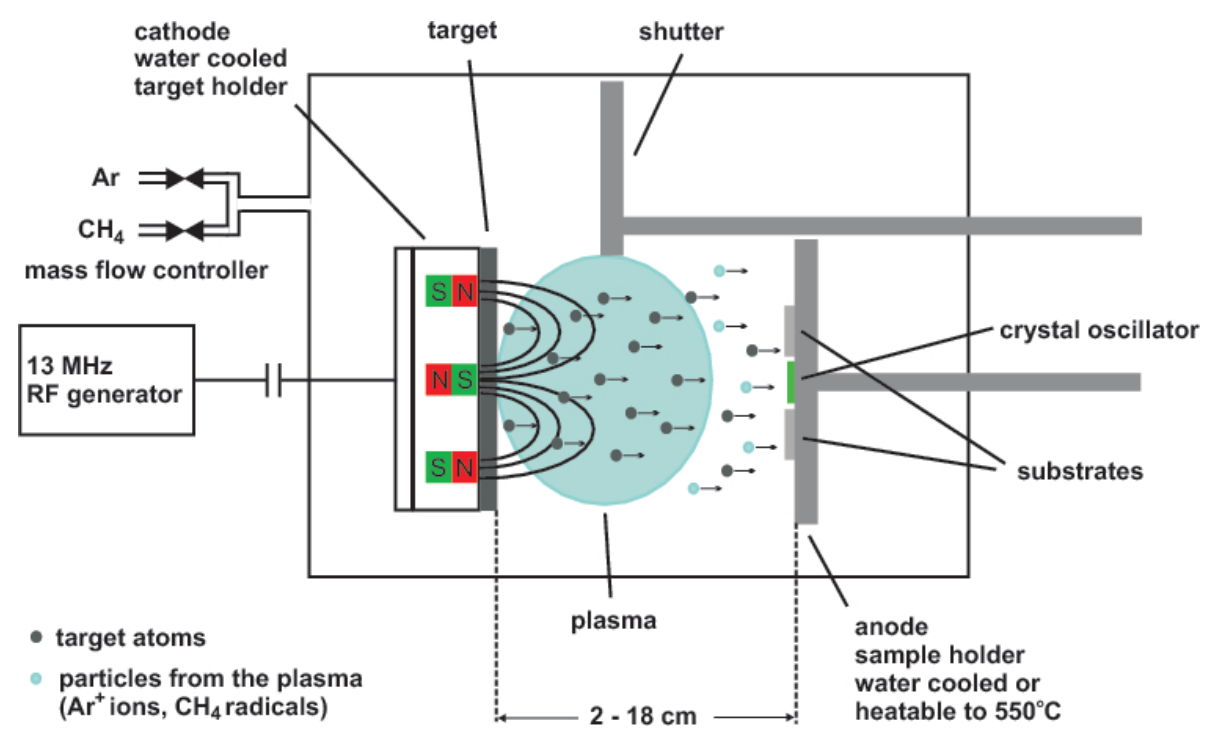

Figure 3.3: Schematic of the R-MSD setup.

\subsection{Reactive Magnetron Sputtering Deposition (R- MSD)}

The second growth method employed in this work is reactive magnetron sputter deposition (R-MSD). On the one hand, this technique provides higher deposition rates than MSIBD, but on the other hand, the deposition parameters cannot be varied independently and are not very well defined.

Figure 3.3 schematically shows the R-MSD setup used. An RF high voltage is applied between the sample holder, which serves as the anode, and the sputtering target holder cathode. The discharge gas is fed into the chamber and a plasma is ignited. The ions from the plasma sputter atoms off the target. Unlike conventional diode sputtering, a magnetic field is applied in the way displayed in Figure 3.3 . Therefore, secondary electrons from the target cathode are forced onto closed trails and the plasma density is enhanced. R-MSD is characterized by a specific fraction of a reactive gas (e.g. an $\mathrm{Ar} / \mathrm{CH}_{4}$ gas mixture).

The energy distribution of the sputtered material is centred at about $0.5-2 \mathrm{eV}$. Ions from the plasma or reflected neutrals from the target arrive at the substrate with energies of about 20-30 eV, corresponding to energies up to the plasma potential [107, 108.

The controllable parameters for R-MSD are the gas composition, the RF power, 
the gas pressure during deposition, the target-substrate distance, and the substrate temperature. While the gas composition mainly effects the final sample composition, the RF power influences the sputtering yield, i.e. the number of particles sputtered off the target and the energy the particle carries after being sputtered. The gas pressure and the target-substrate distance also influence the energy of the impinging particles. At higher pressures, the mean free path of the particles is shorter and therefore the particle energy is reduced due to a larger number of collisions with other gas particles. If the distance between the target and the substrate is decreased, the particles do not have to travel as far and the propability for collisions is reduced.

One problem with reactive sputter deposition techniques can be the coating of the sputter target with atoms from the reactive gas. This effect is known as target poisoning [109]. It results in a reduced content of the sputtered element in the growing film. The deposition parameters can be adjusted to control target poisoning. However, this strongly limits the number of parameter sets and is therefore an unfortunate restriction to the analysis of film structures.

\subsection{MSIBD versus R-MSD Processes}

The processes involved in the two deposition techniques described above show mainly one important similarity, i.e. in all of them the energy of the impinging species is of the same order of magnitude. However, there are a number of differences that can be summed up to the point that the MSIBD parameters are much better defined than the R-MSD parameters.

In MSIBD, the deposited charge can be measured and a well defined fluence ratio of the different elements can be selected by this means. In R-MSD, however, samples of different metal content are grown by varying the $\mathrm{Ar} / \mathrm{CH}_{4}$ ratio, but the final sample composition remains somewhat uncertain and cannot be precisely preset.

In MSIBD, only the selected species, singly charged $\mathrm{C}^{+}$and Metal $\left(\mathrm{Me}^{+}\right)$ions, are deposited with a rather well defined energy. Choosing an ion energy of the order of $100 \mathrm{eV}$ results in an implantation depth of 1-2 $\mathrm{nm}$. The ions are implanted just below the surface or subplanted [110, 111]. The current densities are low and therefore the incident ion beam does not cause any global heating of the substrate. In contrast to this, there are a number of different species at various energies contributing to the growth by R-MSD. From the $\mathrm{Ar} / \mathrm{CH}_{4}$ plasma, $\mathrm{CH}_{x}$ radicals are deposited 
onto the substrate. Ions from the plasma and reflected neutrals impinge on the substrate with energies in the order of the plasma potential with a rather broad distribution centred around 3-20 eV, depending on the deposition pressure and the applied discharge voltage. At these energies, the target atoms may also penetrate as far as 1-2 $\mathrm{nm}$ into the substrate, comparable to the subplantation process in MSIBD. A comparison of the penetration ranges of the respective ions using the Monte-Carlo computer program SRIM [112] (Version 2003) results in only slight differences in the low energy regime. Taking gold ions bombarding an a-C:Au film with a gold concentration of 20 at. $\%$ as an example yields the following: $100 \mathrm{eV} \mathrm{Au}^{+}$ ions penetrate about $1.3 \mathrm{~nm}$ with a straggling of $0.5 \mathrm{~nm}$ while $20 \mathrm{eV}$ ions still have an ion range of $0.9(3) \mathrm{nm}$. Although SRIM was developed for ion implantation, the calculated ion ranges for low-energy ions are reasonably accurate [113. Furthermore, the focus here is not on the exact values but on the comparison, which in the example above results in a factor of only 1.4 in the ion ranges. For the same example a factor of 1.8 for carbon ions with ion energies of $20(0.6 \mathrm{~nm})$ and $100 \mathrm{eV}(1.1 \mathrm{~nm})$, respectively, can be calculated. Recapitulating, a factor of 5 in the ion energy yields only a factor of 1.3-2 in the ion ranges in this low energy regime. Since the absolute values are very small, the differences are just one or two bond lengths [114.

\subsection{Processes During the Deposition of Hyperthermal Species}

The term hyperthermal species was introduced by Lifshitz in 1989 and denotes ions, atoms, or molecules with energies in the range of a few to a few hundred eV [110]. Using them in thin film deposition leads to a number of - partially competingprocesses that differ somewhat from processes during deposition by evaporation. The techniques based on the deposition of hyperthermal species includes, for example sputter deposition, magnetron sputter deposition, laser ablation, ion beam assisted deposition, cathodic arc evaporation, and mass selective ion beam deposition. The comparatively high transfer of kinetic energy and momentum from the incident particle to the substrate or growing film severely influences the final film morphology. Therefore, the qualities of the resulting films may be improved in various ways: higher film hardness, better substrate adhesion, low surface roughness, or epitaxial growth at low temperatures [115, 116, 117]. The use of hyperthermal species can also result in the formation of metastable phases that may not be producible by 
other means (e.g. cubic boron nitride thin films) [118, 119, 120].

In this section, the basic effects of an energetic ion travelling through matter are summarized. Comprehensive early studies on the subject are given by Slater [121] and Seitz and Koehler [122. A thorough review can be found in the book by Nastasi, Mayer, and Hirvonen [123.

An ion impinging on a material with a certain energy loses this energy to the surrounding material until it comes to rest. Generally, there are two ways for the energy to dissipate: by electronic excitation (inelastic scattering) or by nuclear collisions (elastic scattering). As long as the material is conductive, the electronic excitation usually has no permanent effect on the atomic structure of the material. An estimate of the influence of electronic excitations in the case of an insulating material like tetrahedrally bonded amorphous carbon is given by Hofsäss and co-workers [72]: the fraction of an energy of $500 \mathrm{eV}$ deposited in the form of electronic excitation that is transferred to the phonon system is as low as approximately $20 \mathrm{eV} / \mathrm{nm}^{3}$, yielding an energy of $130 \mathrm{meV}$ per atom. If the activation energy for the displacement of an atom is $E^{\prime} \approx 3 \mathrm{eV}$, the contribution from the electron system can be neglected. The energy transfer responsible for changes in the atomic structure is therefore the nuclear elastic scattering process.

In the case of hyperthermal species, where energies are comparatively small and therefore the projectiles are rather slow, the repulsion between the two collision partners is provided by the Coulomb interaction of their electron clouds. This is described by a Thomas-Fermi type screened Coulomb potential ([123], p. 32):

$$
V(r)=\frac{Z_{1} Z_{2} e^{2}}{r} \chi(r),
$$

where $Z_{1}$ and $Z_{2}$ are the atomic numbers of the impinging particle and the target atoms, respectively; $r$ is their distance; $e$ is the elementary charge; and $\chi(r)$ is the screening function. Using this potential and integrating over the product of the energy transfer and the differential cross-section then yields the nuclear stopping power $S_{n}(E)$. With the reduced energy

$$
\epsilon=\frac{a_{T F}}{Z_{1} Z_{2} e^{2}} \frac{M_{2}}{M_{1}+M_{2}} E,
$$

where $a_{T F}$ is the Thomas-Fermi screening length, and $M_{1}$ and $M_{2}$ are the the masses of the impinging ion and the atom in the target, respectively, we get ([123], pp. 9099), 


$$
S_{n}(\epsilon)=\frac{\ln (1+1.1383 \epsilon)}{2\left(\epsilon+0.01321 \epsilon^{0.21226}+0.19593 \epsilon^{0.5}\right)} .
$$

From the stopping power, the mean ion range $r_{I}$ in an amorphous material of atomic density $n$ can now be derived by integration from the initial ion energy $E_{0}$ to the point where the ion is at rest ([123], p. 121):

$$
r_{I}=\int_{E_{0}}^{0} \frac{1}{n S(E)} d E .
$$

The reduced range is related to this ion range by

$$
\rho_{I}=\int_{0}^{\epsilon} \frac{1}{S(\epsilon)} d \epsilon=r_{I} 4 \pi n M_{2} a_{T F}^{2} \frac{M_{1}}{\left(M_{1}+M_{2}\right)^{2}} .
$$

Equations (3.4) and (3.5) are not limited to the low energy regime, where only nuclear stopping is relevant. However, for small energies, the stopping power is $S(E) \propto \sqrt{E}$ and the integration yields $r_{I} \propto \sqrt{E}$, as is in accordance with the values calculated using the Monte Carlo program SRIM [112] in the previous section. Of course, the ion range does not take discrete values, but can rather be described by a Gaussian distribution with straggling in the $x y$-plane if the surface normal is in $z$-direction.

The basis of the Monte Carlo simulation program SRIM for the calculation of mean ion ranges is a linear collision approximation using empirical screened Coulomb potentials similar to the one given in Equation (3.1) [112] and semi-experimental stopping powers $S_{e} l$. In the case of $100 \mathrm{eV} \mathrm{C}^{+}$ions impinging on either a-C or a$\mathrm{C}: \mathrm{Me}(\mathrm{Me}=\mathrm{Cu}, \mathrm{Ag}, \mathrm{Au}, \mathrm{Fe}$; metal concentration: 20 at.\%), the ion ranges calculated by SRIM result in values between $0.9(4) \mathrm{nm}$ and 1.1(6) nm. For the metal ions $(40 \mathrm{eV}$ $\mathrm{Fe}^{+}, 40 \mathrm{eV} \mathrm{Cu}^{+}, 60 \mathrm{eV} \mathrm{Ag}^{+}$, and $100 \mathrm{eV} \mathrm{Au}^{+}$), the ion ranges in a-C lie between $0.8(1) \mathrm{nm}$ and 1.6(1) $\mathrm{nm}$. For the same ions impinging on the respective metalcontaining a-C, with a metal concentration of 20 at.\%, the values range between $0.7(2) \mathrm{nm}$ and $1.3(5) \mathrm{nm}$. The implantation profile of $100 \mathrm{eV} \mathrm{Au}$ on a film of 80 at.\% carbon and 20 at.\% gold is shown in Figure 3.4 .

The process of energy transfer from the incoming particle to the surrounding material can be divided into three regimes that occur at rather different time scales: the collision cascade, the thermal spike, and the relaxation stage. Within the first $10^{-15} \mathrm{~s}$, the impinging ion is scattered by the atoms in the solid. If an atom is released from its site in such a binary collision, it travels at a velocity according to 


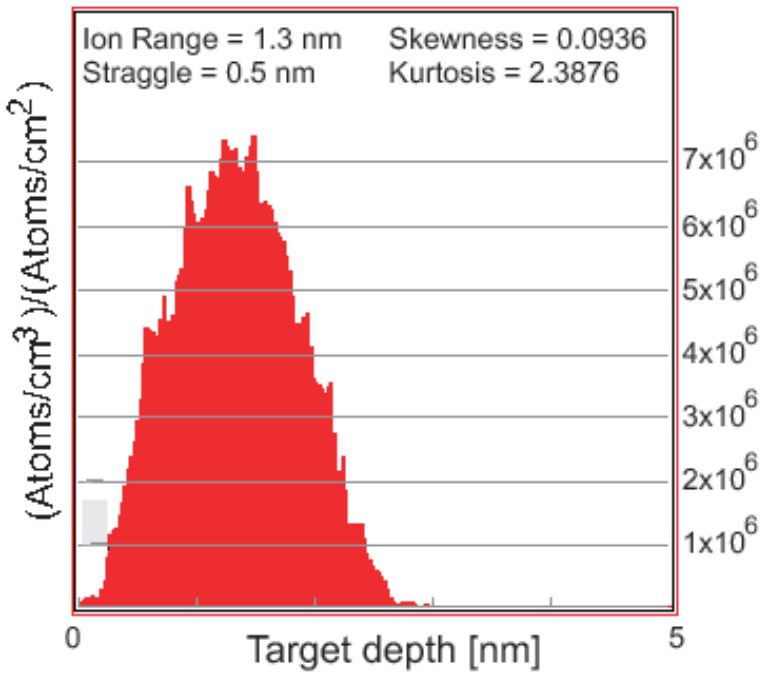

Figure 3.4: SRIM simulation of the implantation profile of $100 \mathrm{eV}$ $\mathrm{Au}^{+}$ions implanted into a layer of 80 at.\% carbon and 20 at.\% gold 112 .

the residue of the energy it received minus the bonding energy. If this remaining energy is small, the released target atom does not impose any further damage to the target and we talk about primary displacements. If it is large enough, it may release further atoms from their sites. Therefore, secondary or even higher order displacements are possible, and the result is a collision cascade. In this time regime, also phonons are excited.

On a time scale of $10^{-11} \mathrm{~s}$, the generated phonons dissipate. According to Seitz and Koehler [122], the portion of the energy transmitted to the lattice by an incident particle results in very concentrated lattice vibrations yielding extremely high local temperatures. The affected volume is called a thermal spike and resembles a liquid, in which a number of atoms move freely until the material freezes in a rearranged atomic structure. In the case of a low energetic ion impinging on a film, the geometry of the spike is assumed to be cylindrical rather than spherical. The energy is released radially from the initial path of the ion. The cylindrical thermal spike is treated theoretically in Reference [124]. The total number of displaced atoms in a cylindrical thermal spike is derived from the assumption that the deposited energy produces a localized increase in temperature that spreads and dissipates according to classical heat conduction in a continuum.

After the collision cascade and thermal spike stages, the material relaxes until the next particle arrives in the same surroundings. During this stage, diffusion processes, chemical reactions, phase transformations, or stress relaxation occur. Depending on the current densities, this may take up to milliseconds.

The displacement of atoms from their original sites can have various wanted or 
unwanted effects: in a crystalline material, defects are induced, surface atoms may desorb (sputtering), mixing of interfaces may occur, the material may be transformed to a metastable phase, and radiation-triggered diffusion may occur. In this thesis, all of these effects play a certain role. First, the silicon substrates of the MSIBD-grown films are pre-treated with a $1 \mathrm{keV} \mathrm{Ar}^{+}$ion beam in order to sputter off the $\mathrm{SiO}_{2}$ layer and amorphize the material. Second, during analyses that employ ion beams in order to deliberately sputter the film for depth profiling, a multilayer structure may be blurred. Third, the aim is to grow the matrix with a high content of $\mathrm{sp}^{3}$ bonds. Fourth, if enough energy is transferred to a surface atom it may overcome the binding to the film and will desorb. This can influence the final film composition. And fifth, metal atoms are transported through the matrix. In the following, the last three points will be discussed in more detail as well as the enhancement of surface diffusion.

\section{The Generation of $\mathrm{sp}^{3}$ Bonds During Ion Beam Deposition}

In their publication on the cylindrical thermal spike model, Hofsäss and co-workers examined the influence of the ion energy on the $\mathrm{sp}^{3}$ bonding content of amorphous carbon films [72]. They regard a Gaussian energy density rather than a temperature in the spike volume and consider only the energy $Q$ available for phonon excitations. The energy dissipation is regarded as a transport process with a diffusion constant $D$ determined by the velocity of sound and the phonon mean free path. The number of rearrangement processes $n_{T}$ can be calculated and compared to the number of atoms in the spike volume $n_{S}$. The length $L$ and width $\sigma$ of the thermal spike is assumed to be given by the mean ion range and the width of the cylindrically symmetric Gaussian energy density distribution, respectively. Rearrangement processes require an activation energy $E^{\prime}$ and occur at a rate $\nu=\nu_{0} e^{E^{\prime} / k T}$, where $\nu_{0}$ is the attempt frequency, which is of the order of phonon frequencies. These assumptions yield

$$
\frac{n_{T}}{n_{S}} \approx\left(\frac{Q}{L}\right)\left(\frac{1}{E^{\prime}}\right) \frac{e \nu_{0}}{4 \pi n D} \exp \left(-\sigma^{2} / \sigma_{c}^{2}\right)
$$

for the fraction of rearrangement process of available atoms in diamond-like carbon materials. In Equation 3.6), $n$ is the atomic density and $\sigma_{C}=\sqrt{Q / 2 \pi E^{\prime} N L}$ denotes a characteristic width of the thermal spike. Using this, Hofsäss and co-workers found an optimum ion energy of $100 \mathrm{eV}$ for the generation of the maximum $\mathrm{sp}^{3}$-bonding content in amorphous carbon [72]. 


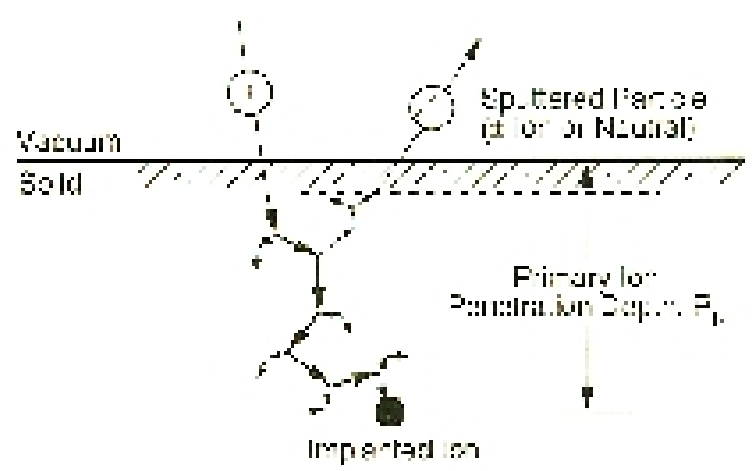

Figure 3.5: $\quad$ Schematic of linear collision cascade sputtering (from [123]).

\section{Sputtering}

The sputtering yield $s$ is defined by the mean number of eroded atoms per incoming particle. The incident ion transfers energy to atoms in the target which may transfer energy to other target atoms. If the recoil energy of an atom at the surface due to a collision is large enough to overcome the surface binding energy, it is released from the target. A possible collision cascade leading to the sputtering of an atom is schematically shown in Figure 3.5. The sputtering yield is mainly influenced by the amount of energy deposited into the region near the surface, $F_{D}\left(E_{0}\right)=\alpha n S_{n}\left(E_{0}\right)$, where $\alpha$ is a correction factor that is a function of $M_{2} / M_{1}$ and accounts for the angle of incidence of the beam to the surface. It furthermore depends on the material factor $\Lambda$, which contains the surface binding energy $U_{0}$, etc. ([123], pp. 218-223):

$$
s=\Lambda F_{D}\left(E_{0}\right) .
$$

According to Sigmund's description (Chapter 2 by P. Sigmund in Reference [125]), the material function is $\Lambda \approx 4.2\left(n U_{0}\right)^{-1} \mathrm{~nm} / \mathrm{eV}$. Therefore, the sputtering yield can be written as

$$
s \approx 4.2 \alpha S_{N}\left(E_{0}\right) / U_{0} .
$$

The linear collision cascade approximation does not apply to the deposition of low-energy ions, because the sputtering yield is strongly enhanced by the thermal spike close to the surface ([123], pp. 244-247). The atoms in a cylindrical volume can be treated as an ideal gas with a Maxwellian velocity distribution and a mean velocity $\bar{v}=\sqrt{\frac{k_{B} T_{\text {surf }}}{2 \pi M_{2}}}$. The spike surface temperature $T_{\text {surf }}$ is proportional to the average deposited energy at the surface $E_{\text {surf }} \propto S_{n}(E) / A_{\text {cas }}^{\text {surf }}$, where $A_{\text {cas }}^{\text {surf }}$ is the spike surface area emitting particles. The evaporation rate per unit time and unit 
area is

$$
J_{e} \approx n \bar{v} \exp \left(\frac{-U_{0}}{k_{B} T_{\text {surf }}}\right) .
$$

For a spike lifetime $\tau$, the sputtering yield is then given by

$$
s_{\text {spike }}=\tau A_{\text {cas }}^{\text {surf }} J_{e} .
$$

A comprehensive description of sputtering is found in the books edited by Behrisch [125, 126, 127].

The MC simulation program SRIM makes use of the binary collision model for the calculation of sputtering yields. The thus obtained sputtering yields can be compared with the sputtering yields gained from experiments. In the case of the MSIBD-grown films, the experimental sputtering yields are easily accessible from the comparison of the metal ion fraction during deposition with the actual concentrations obtained from composition analysis methods, such as Rutherford Backscattering Spectroscopy.

\section{Ion Beam Induced Atomic Transport}

The cylindrical thermal spike model can also be used to estimate the ion-induced transport of atoms within the film. The displacement and rearrangement is, of course, a means of transportation. The number of displacements arising from the collisional stage is negligible in comparison to the rearrangement during the thermal spike for the low ion energies employed in this thesis [72]. We express the number of atoms rearranged in a thermal spike, as it was calculated by Vinyard [124], in correspondence with the cylindrical thermal spike model by Hofsäss and co-workers [72] to

$$
n_{T}=\frac{\nu_{0} \varepsilon^{2}}{8 \pi \kappa C E^{\prime 2}}
$$

where $\nu_{0}$ is the jump attempt frequency, $\varepsilon$ is the energy density per unit length introduced at time $t=0$ along the centre line of the cylindrical spike, and $\kappa$ and $C$ are the temperature-independent thermal conductivity and heat capacity of the material, respectively [124]. If the fraction $n_{T} / n_{S} \approx 1$ (see Equation (3.6)), each atom within the surrounding of the ion path moves the distance of about 1 atom spacing per incoming ion. This results in an overall transport of atoms with travelled distances in the order of a few nanometres. 


\section{Ion Beam Enhanced Surface Diffusion}

The enhancement of surface diffusion under the bombardment by energetic particles has been observed in various experiments, but is nevertheless not fully understood yet. Thermally activated surface diffusion proceeds according to Fick's law $\vec{\Gamma}=$ $-D \vec{\nabla} c$, where $\vec{\Gamma}$ is the flux of the diffusing species, $c$ denotes the concentration, and $D=D_{0} \exp \left(-E_{d} / k_{B} T\right)$ is the diffusion coefficient with the activation energy $E_{d}$ for diffusion (see e.g. Reference [129]).

The most common influence of an ion impinging on a surface is given by its collision with a surface atom. The probability of this atom to diffuse is then significantly enhanced, as more energy is transferred from the ion to the atom $E_{i \rightarrow a}$. This is introduced by lowering the activation energy [130]:

$$
P \propto \exp \frac{-\left(E_{d}-E_{i \rightarrow a}\right)}{k_{B} T} .
$$

Furthermore, as discussed above, low-energy particle bombardment leads to thermal spikes in the region near the surface. The local increase in temperature $\delta T$ due to the spike should also enhance the surfaces diffusion. The diffusion constant yielding the combined effect of thermally activated diffusion plus the enhancement due to the thermal spike is then [129]:

$$
D=D_{0}\left[\exp \frac{-E_{d}}{k_{B} T}+f_{\text {spike }, t} \exp \frac{-E_{d}}{k_{B}(T+\delta T)}\right]
$$

where $f_{\text {spike, } t}$ gives the fraction of the surface that is in the state of a thermal spike at any given time. In particular, $f_{\text {spike, } t}$ only depends on the current density of the bombarding ions and not on the temperature. Therefore, from Equation (3.13) it is expected that the relative increase of diffusion due to an increasing current density will be larger for lower substrate temperatures. However, the temperature dependence found in experiments is just the opposite. Robinson and Rossnagel therefore imply that the enhancement of surface diffusion should be related to the increased number of ion beam excited phonons from the ion impact [129]. As their relaxation to lower energy phonons is comparatively slow, they have an effect over larger areas and interaction between multiple ion impacts may occur. This process implies the same temperature dependence as suggested above. It is therefore suggested that a minimum ion-induced phonon density in the surface regions may be required for the radiation enhanced surface diffusion. 


\section{Chapter 4}

\section{Characterization Techniques}

Several characterization methods were implemented in order to obtain a good understanding of the structural properties of the films and the mechanisms by which these structures evolve. This chapter gives a short overview of the basics of these techniques.

\subsection{Transmission Electron Microscopy and Energy Dispersive X-Ray Spectroscopy}

The most powerful tool for the analysis of film morphologies is transmission electron microscopy (TEM). As in nanoscience ordinary light microscopy fails due to its limited resolution, microscopes offering radiation of much shorter wavelengths are essential. However, the theoretically feasible resolution as determined by Abbe's formula, when applying high energy electrons, cannot be reached in actual electron microscopes. The resolution is rather limited because of the poor quality of the electron lenses, which is often compared to the quality of using the bottom of a coke bottle as a magnifying glass [131]. Reference [131] is also an excellent textbook dealing with most aspects of transmission electron microscopy.

\section{Specimen Preparation}

Electrons are easily absorbed by matter. Therefore, a sample has to be thinned extensively, before it is suitable for TEM analysis. Especially for amorphous materials, the sample thickness has a strong influence on the resolution that can be obtained. Using specimens thinned perpendicular to the film surface allows a cross-sectional view of the sample and therefore the analysis of its vertical structure. 


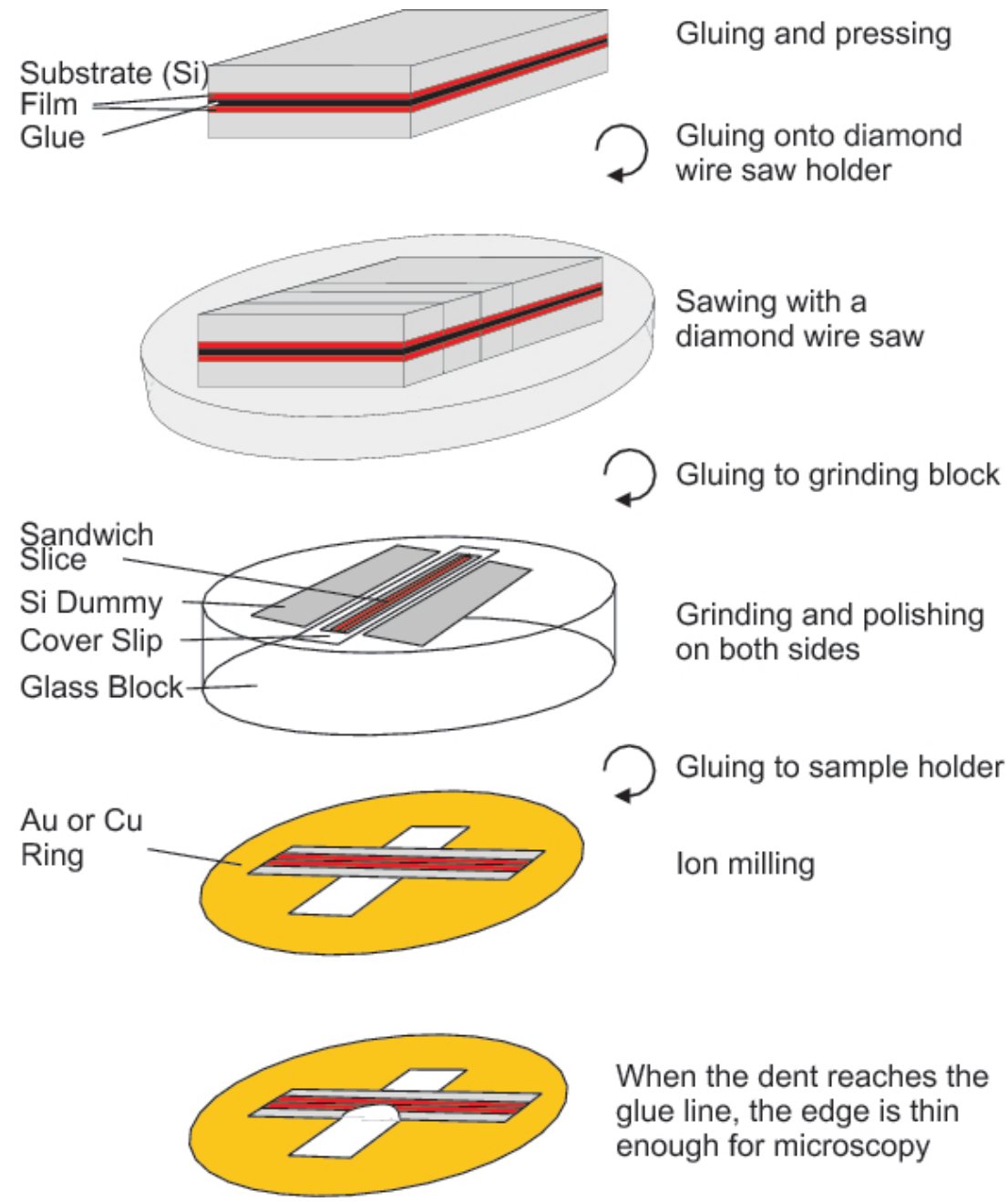

Figure 4.1: The TEM sample preparation process (not to scale).

Before preparing a sample for TEM analysis, a few questions have to be considered: how does the film respond to certain solvents? Is the film well adherent to the substrate? Is it sensitive to thermal treatment? Considering the ion-beam deposited samples, the answer to the first two questions is positive: the films are chemically inert and withstand any contact with conventional solvents. Furthermore, the film adhesion is rather good due to the sub-surface growth of the films and a pre-deposition Argon sputtering process, which leaves a pure amorphous silicon substrate to deposit onto. However, previous annealing experiments showed that the films may undergo changes in composition and structure, even at temperatures as low as $150{ }^{\circ} \mathrm{C}$. Therefore, the TEM specimen preparation has to be performed at 
room temperature. Only glues that do not need heating as a hardening process or that do not have to be melted can be used. The preparation mechanism employed for the samples discussed in this thesis is shown in Figure 4.1 and will be outlined in the following.

- Making a sandwich: in the first step, a homogeneously coated part is cut from the substrate using a diamond scriber. The resulting piece is cut in half. The two parts are cleaned in an ultrasonic bath and glued together with the coated sides facing one another, comparable to a sandwich (bread=siliconsubstrate, butter $=$ film, cheese $=$ glue). The glue used for sandwiching is a $U H U$ Endfest 300 Plus two-component adhesive.

- Preparing a number of slices: the sandwich is glued to a ceramic block using UHU Sekunden-Alleskleber. The ceramic block serves as a sample holder for a diamond wire saw. It allows accurate sawing of the sandwich into a number of slices, each of $0.5 \mathrm{~mm}$ width. Afterwards, the slices are removed from the holder by bathing in acetone. Only one slice is necessary for the following steps. However, a slice may easily break in the process. In that case, one of the additional slices serves as a backup.

- Grinding and polishing: one of the slices is glued onto a $150 \mu \mathrm{m}$ thick cover slip on a glass block of known thickness. The glue line is now vertically oriented. Two pieces of silicon (silicon dummies) are glued on either side of the cover slip. They serve as protection and allow the measurement of the current thickness of the sandwich slice. The silicon dummies and the cover slip are glued to the glass block using an epoxy resin with a melting point of $150^{\circ} \mathrm{C}$. The glue used to fix the sandwich slice onto the cover slip is again UHU Sekunden-Alleskleber. The sample is ground using abrasive paper with granularities P 500 and P 1000 (Fepa) to about half its original thickness. In the next step, the sample is polished with Winter Diaplast polishing paste of $7 \mu \mathrm{m}, 1 \mu \mathrm{m}$, and $0.25 \mu \mathrm{m}$ diamond grains. The sample is then removed from the holder in an acetone bath, turned over and glued back onto the cover slip. Now, the other side is ground and polished until a final thickness of $\leq 30 \mu \mathrm{m}$ is reached. At this stage, the sample shimmers in a reddish tone when observed in the dark field mode of an ordinary light microscope. Again, the slice is removed from the holder in an acetone bath. 
- Attaching the sandwich slice to a support ring: the slice has to be glued onto a support ring, usually made of copper, gold, or nickel. The ring has a suitable diameter and thickness to fit into regular TEM sample holders. It is important to choose a ring material that is not contained in the film to be analyzed.

- Ion milling: the sample is now thinned by ion milling in a Gatan dual ion mill; first under an angle of $17^{\circ}$ at $4 \mathrm{kV}$ and $1 \mathrm{~mA}$. When the sandwich shows a dent, the angle, voltage, and current are reduced to $12^{\circ}, 3 \mathrm{kV}$, and $0.8 \mathrm{~mA}$, respectively. The sample is sufficiently thinned when the dent in the slice has reached and cut the glue line. The sample holder is mounted on a pole that is cooled with liquid nitrogen during the entire ion milling process.

Three samples were prepared for cross-sectional TEM using the focused ion beam setup Nova 600 Nanolab. In order to obtain electron transparent lamellae, the following procedure is implemented: first, two layers of platinum of about $3 \mu \mathrm{m}$ overall thickness are grown by electron beam and ion beam assisted chemical vapour deposition from an organometallic gas. In the next step, a lamella about $5 \mu \mathrm{m}$ in width, $7 \mu \mathrm{m}$ in depth, and $1 \mu \mathrm{m}$ in thickness is cut free by using $30 \mathrm{kV}$ focused $\mathrm{Ga}^{+}$ ions. Only one small bridge connects the lamella to the surrounding material. It is then fastened to an Omniprobe nanomanipulator by platinum deposition and the connection to the material is cut by the focused ion beam. From here, it is fastened to a support that fits into regular TEM sample holders by platinum deposition. The connection to the manipulator is cut by focused ion beam sputtering. Finally, the lamella is thinned to a thickness of a few nanometres by $30 \mathrm{kV}$ and $5 \mathrm{kV} \mathrm{Ga}{ }^{+}$ ion sputtering. A comprehensive description of TEM sample preparation using a focused ion beam is given in Reference [132].

\section{The Microscope Philips CM 200-UT}

The Philips CM 200-UT of the $4^{\text {th }}$ Institute of Physics of the University of Göttingen was used for all TEM analyses reported on in this thesis. This microscope combines a field emission electron source with a high-resolution objective lens. Therefore, the best point resolution is $0.187 \mathrm{~nm}$ and the smallest distance that can be resolved is $0.11 \mathrm{~nm}$. In addition to conventional TEM and high resolution TEM (HRTEM), this microscope can be operated in scanning mode. It is equipped with an X-ray 
detector, in order to allow energy dispersive X-ray spectrometry (EDX). This is a very convenient tool for a chemical analysis at specific locations of the film with a resolution of down to 1-2 nm. For instance, the chemical composition can be obtained along a line across the film (line scans). These line scans are of great value for the analysis of multilayer structured films.

\subsection{Rutherford Backscattering Spectroscopy}

In order to determine a sample's integral elemental composition and obtain a concentration depth profile, Rutherford backscattering spectroscopy (RBS) is a very convenient tool. Figure 4.2 shows the principle of an RBS measurement. A beam of light ions (e.g. $\mathrm{He}^{2+}$ ) is focused onto the sample. The particles are backscattered due to elastic scattering by the target nuclei and therefore suffer a characteristic transfer of energy, depending on the mass of the nuclei they were scattered by. The energy loss due to this process is expressed by the $K$-factor, which gives the ratio of the projectile energy before and after scattering:

$$
K:=\frac{E_{P}}{E_{0}}=\left(\frac{\sqrt{1-\left[\left(M_{P} / M_{T}\right) \sin \theta\right]^{2}}+\left(M_{P} / M_{T}\right) \cos \theta}{1+\left(M_{P} / M_{T}\right)}\right)^{2},
$$

where $E_{0}$ and $E_{P}$ are the energies of the incident and backscattered projectile, respectively; $M_{P}$ and $M_{T}$ are the masses of the projectile and the target atom, respectively; and $\theta$ is the scattering angle. The $K$-factor value is characteristic to $M_{T}$ for fixed $E_{0}, M_{P}$ and $\theta$. Therefore, it is possible to identify the elements in the target and gain information about the sample composition. This technique is

Figure 4.2: The principle of Rutherford Backscattering Spectroscopy: A beam of particles of the mass $M_{P}$ is focused onto the target at an energy $E_{0}$. The incoming projectiles are backscattered by the target atoms of mass $M_{T}$ and therefore encounter a characteristic loss of energy in addition to the electronic energy loss depending on the incoming and outgoing path length. The energy of the backscattered projectile $E_{P}$ is measured by the detector.

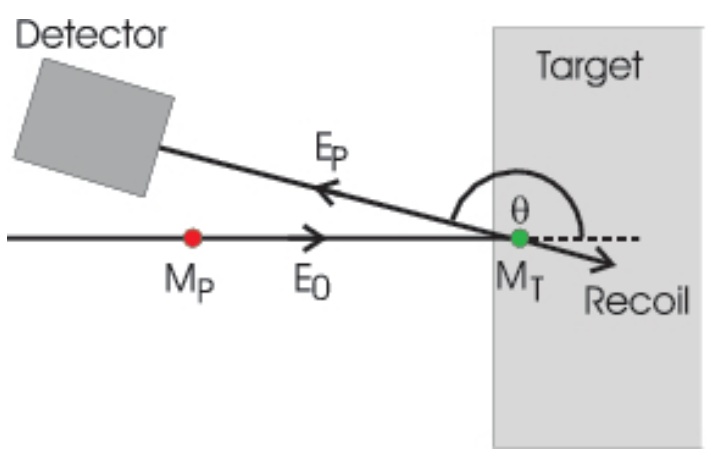




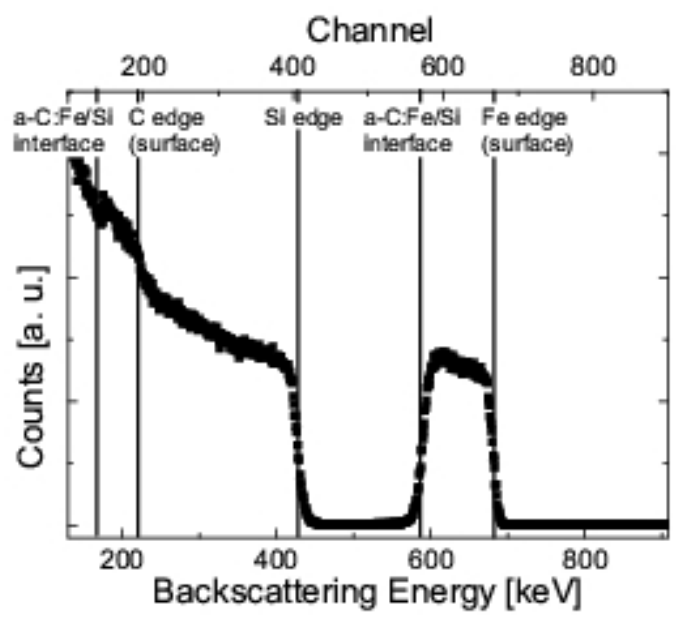

Figure 4.3: Example of a typical RBS spectrum of an a-C:Fe film of about $120 \mathrm{~nm}$ thickness on a silicon substrate. The incident particles are $\mathrm{He}^{2+}$ ions with an energy of $900 \mathrm{keV}$.

especially applicable for the analysis of heavy elements in light matrices, such as amorphous carbon.

If the incoming projectile is not backscattered at the surface of the film, it will suffer a loss of energy while travelling through the material. Therefore, a particle scattered at some depth also carries a smaller energy than a particle scattered at the surface. One obtains a box-shaped signal. Light elements incorporated in a thin film on top of a substrate of heavier elements will result in an overlap of the sample signal and the substrate signal. A representative spectrum of an a-C:Fe film grown on a silicon substrate is given in Figure 4.3. The carbon signal and the silicon substrate signal are superimposed. The large signal with its surface edge at about $680 \mathrm{keV}$ arises from the ions backscattered from iron atoms. The areal density of the incorporated metal can be obtained from the integral over its RBS signal if the Rutherford scattering cross-section in the centre-of-mass frame is considered:

$$
\frac{d \sigma}{d \Omega}=\left(\frac{Z_{P} Z_{T} e^{2}}{16 \pi \epsilon_{0} E_{0}}\right)^{2} \frac{1}{\sin ^{4}(\theta / 2)} .
$$

Here, $Z_{P}$ and $Z_{T}$ are the atomic numbers of the incoming projectile and the target atom, $E_{0}$ and $\theta$ denote the same quantities as above, and $e, \pi$, and $\epsilon_{0}$ have their usual meaning. If $N$ is the number of counts integrated from the metal signal, $Q$ is the number of impinging ions, and $\Delta \Omega$ is the detector opening angle, then the 
areal density $n$ is given by

$$
n=\frac{N}{Q \frac{d \sigma}{d \Omega} \Delta \Omega}
$$

The areal density of a given element in the ion beam deposited films can then be compared to the fluence of the respective ion species during deposition. Analysis of the integral concentration or, where applicable, investigation of the concentration depth profile, was performed using the software packages RUMP and NDF [133, 134]. A general introduction to RBS is given in Reference [135.

RBS was performed at the Göttingen heavy ion implanter IonAS using $900 \mathrm{keV}$ $\mathrm{He}^{2+}$ ions [136]. The RBS chamber is equipped with three silicon surface barrier detectors with an opening angle $\Delta \Omega=3.4 \mathrm{mSr}$. The detectors are placed at an angle of $\theta=165^{\circ}$ to the incident $\mathrm{He}^{2+}$ beam. The depth resolution depends on the energy resolution of the detectors, which is typically $12.5 \mathrm{kV}$ in the present setup.

\subsection{Raman Spectroscopy}

The Raman effect was first predicted by Smekal in 1923, and in 1928 Raman succeeded in its experimental verification [137, 138]. An incident laser beam aimed at a sample interacts with the molecular vibration modes or phonons. If inelastic scattering occurs, the light may either loose or gain energy according to the quantized states of these vibrations (Stokes and anti-Stokes scattering). The case of energy loss to the sample vibrations has a much higher probability, as mostly the vibrational ground states are occupied at moderate temperatures. The energy shift (or shift in wavenumbers) after inelastic scattering from the sample gives insight into its atomic structure. Reference [139] is a good textbook introducing theoretical and practical aspects of Raman spectroscopy.

There are several methods suitable for the analysis of the $\mathrm{sp}^{3}$-bonding fraction of an amorphous carbon film, and visible Raman spectroscopy is not the most accurate one of these. However, it becomes invaluable, as the other methods, for example electron energy loss spectroscopy or X-ray photoelectron spectroscopy are very surface-sensitive and are only reasonable if the measurements can be performed without breaking the vacuum. Furthermore, these methods may yield wrong results, when the films to be analyzed are not pure carbon films.

The Raman spectra of different carbon phases are shown in Figure 4.4. The Raman line of diamond at $1331 \mathrm{~cm}^{-1}$ arises from the transverse optical phonon. 
(a)

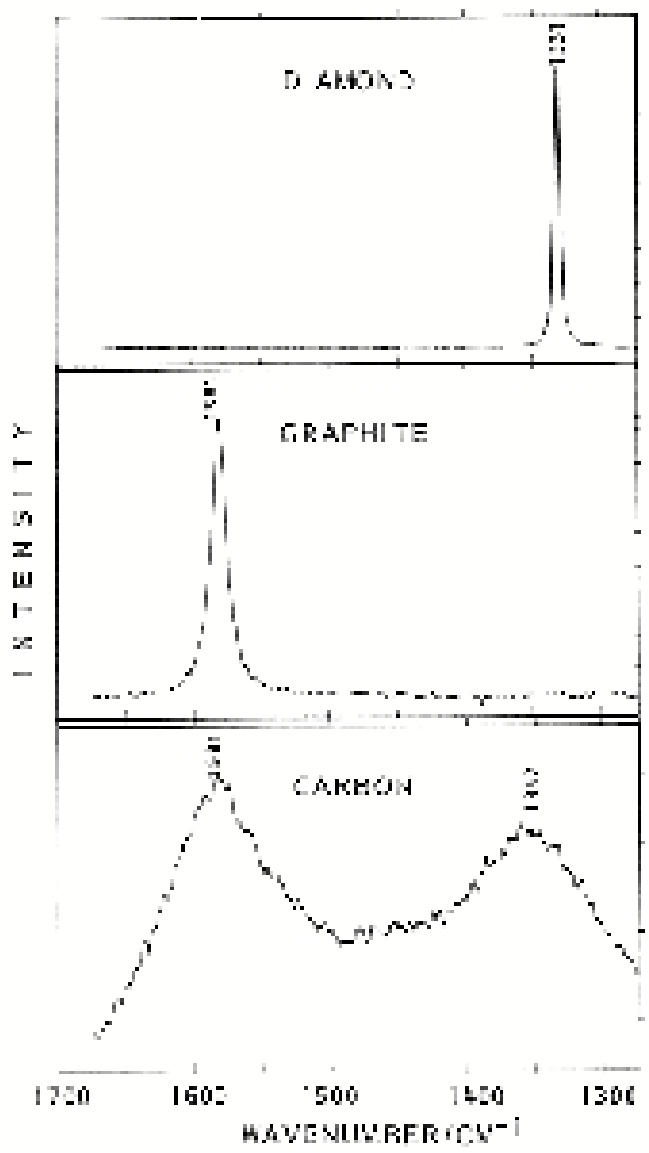

(b)

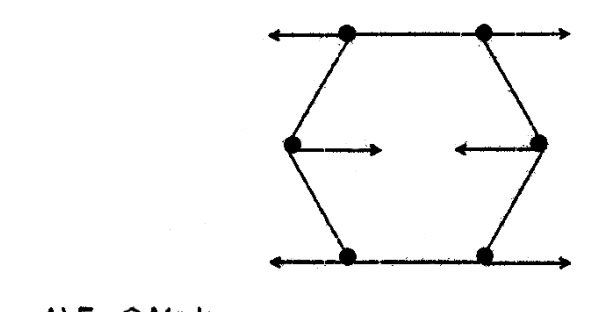

A) $E_{20} G$ Mode

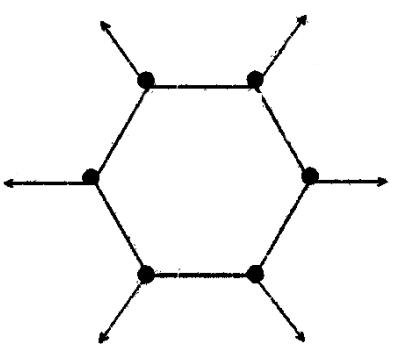

B) $A_{19} D$ breathing mode

Figure 4.4: (a) Raman spectra of diamond, HOPG, and disordered carbon. In the spectrum of disordered carbon, the G-band is situated at $1581 \mathrm{~cm}^{-1}$ and the D-band at $1352^{-1}$ (from [140]). (b) Vibration modes of graphite rings (from [141]).

Highly ordered pyrolytic graphite vibrates in $\mathrm{E}_{2 g}$ symmetry resulting in a Raman shift of $1580 \mathrm{~cm}^{-1}$. When the aromatic rings of graphite are disordered, this peak significantly broadens into the (G-band), and the $\mathrm{A}_{1 g}$ breathing mode becomes active and gives rise to a further signal at $1352 \mathrm{~cm}^{-1}$ (D-band) [140, 141].

In highly tetrahedrally bonded amorphous carbon, there are virtually no complete aromatic rings present. Therefore, the D-band vanishes from the spectrum and leaves a slightly skew broad peak. The intensity distribution of this peak can best be fitted using a Breit-Wigner-Fano (BWF) function [143]

$$
I(\omega)=\frac{I_{0}\left[1+2\left(\omega-\omega_{0}\right) / Q \Gamma\right]^{2}}{1+\left[2\left(\omega-\omega_{0}\right) / \Gamma\right]^{2}}
$$


Figure 4.5: Raman spectra of a-C films containing different fractions of $\mathrm{sp}^{3}$ bonds (indicated near each spectrum). The spectra were obtained with an excitation wavelength of $244 \mathrm{~nm}$ (from [142]).

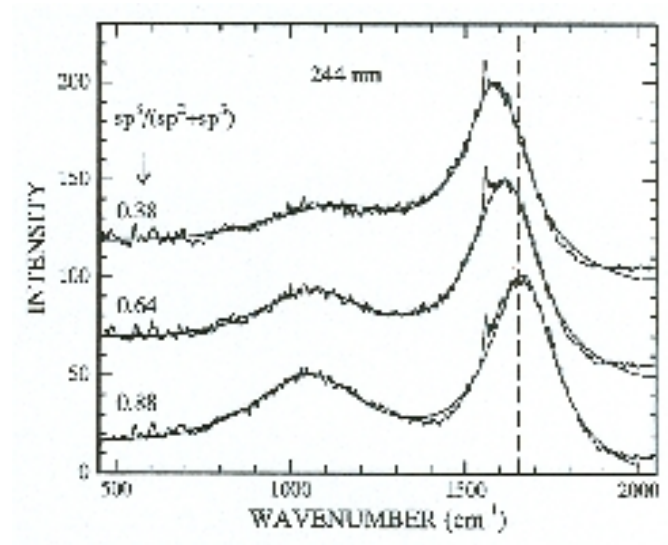

Here, $I_{0}$ is the line intensity, $\omega$ is the angular frequency of the scattered light, $\omega_{0}$ is the peak position in the spectrum, $\Gamma$ is the full width at half maximum, and $Q$ is the BWF coupling parameter. If $1 / Q \longrightarrow 0$, Equation (4.4) approaches a Lorentzian line shape and the sample has a high $\mathrm{sp}^{3}$-bonding content. However, $Q$ has no physical meaning. As the $\mathrm{sp}^{2}$-bonding content increases, the signal becomes more skew and the D-band gains in intensity an additional Lorentzian is used to fit the spectrum.

A few publications promote a quantitative analysis of the $\mathrm{sp}^{3}$ content of a film by calculating the ratio of the intensities of the $\mathrm{G}$ and D-band and observing peak positions and the coupling parameter $Q$ [141, 144]. However, this procedure seems to be suitable only under certain growth conditions and a comparison with more reliable techniques shows that the method should rather be regarded as a means of gaining relative and qualitative information.

More reliable information can be gained if UV Raman is available [142. As described above, the $\mathrm{G}$ and D-band in the spectra gained by visible Raman spectroscopy are both generated by vibrations of $\mathrm{sp}^{2}$ bonds. The commonly used 488 and $514.5 \mathrm{~nm}$ lines of Ar ion lasers energetically correspond to $\pi-\pi^{*}$ transitions at $\mathrm{sp}^{2}$ sites. Therefore, the Raman signal of the $\mathrm{sp}^{2}$ bonds is resonantly enhanced. The spectrum is dominated by the $\mathrm{G}$ and $\mathrm{D}$-band described above. The vibrational mode of the $\mathrm{sp}^{3}$ bonds, positioned at about $1100 \mathrm{~cm}^{-1}$, cannot be seen, as the G-band is about two orders of magnitude stronger. Using a UV laser of $244 \mathrm{~nm}$ wavelength for excitation, this band becomes visible (see Figure 4.5). Nevertheless, even a film with $88 \% \mathrm{sp}^{3}$ bonds still shows a strong G-band at about $1650 \mathrm{~cm}^{-1}$.

The Raman spectra presented in this thesis were obtained with two different setups. The first is the Jobin Yvon T 64000 of the Institute of Physical Chemistry, 
Department of Chemistry. This spectrometer can be operated in micro mode or in macro mode. It is equipped with a three stage monochromator and a $2000 \times 800$ pixel CCD camera. The excitation laser is an argon ion laser. Spectra were recorded using an excitation wavelength of $488 \mathrm{~nm}$. The second spectrometer is a Dilor XY at the $4^{\text {th }}$ Institute of Physics of our Department. It is also equipped with a three stage monochromator and a CCD camera. In this case, the excitation wavelength of $514.5 \mathrm{~nm}$ of an argon ion laser was aimed at the sample at grazing incidence.

\subsection{X-Ray Diffraction}

The aim of using X-ray diffraction (XRD) for this thesis was twofold: it is, of course, a powerful tool for the analysis of different phases in a film, but can also yield a mean particle size of small crystal grains within a sample. Employing the powder method to a film with randomly oriented crystals will result in a ring-shaped diffraction pattern, as the X-rays diffracted according to Bragg's law will form a cone when leaving the sample. The width of the intensity profile of such a diffraction ring gives insight into the mean size of the particles that the incident beam was diffracted by. An infinite crystal results in an intensity profile of zero width, because for every X-ray scattered under an angle only infinitely smaller or larger than the Bragg angle there is an X-ray scattered at a lattice plane in such a way that the phase difference is $\lambda / 2$. The smaller the crystal, the larger the angular interval contributing to the diffraction signal. The mean particle diameter can therefore be calculated from the Lorentzian line width gained from the deconvolution of the Voigt function used to

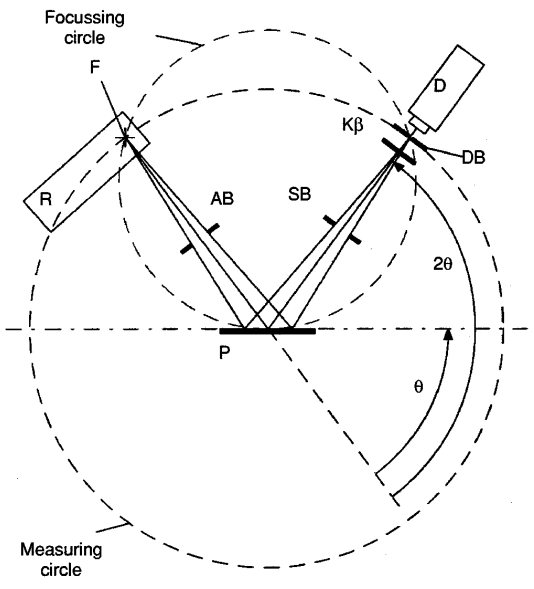

Figure 4.6: Schematic of the XRD setup: coming from the focus (F) of the X-ray source $(R)$, the incident beam passes an aperture $(\mathrm{AB})$ and is aimed onto the sample $(\mathrm{P})$. The diffracted beam passes a scattering light aperture, a $\mathrm{K} \beta$ filter and a detector aperture before entering the detector. This schematic also illustrates the $\theta / 2 \theta$-method described in the text (from [146]). 
fit the diffraction signal according to the Scherrer formula [145]:

$$
t=\frac{0,9 \lambda}{B \cos \theta}
$$

This rather powerful method is mainly limited by the small intensities gained from a too small number of diffraction sites. It can only be reasonably employed if either rather thick films are investigated or the sample obtains a high fraction of the crystalline phase.

The diffractometer used here was a Bruker AXS equipped with a $\mathrm{Cu} \mathrm{K} \alpha$ source $(\lambda=1.54 \AA)$. The setup is schematically illustrated in Figure 4.6 . Two measuring modes were important for this thesis: $\theta / 2 \theta$ and grazing incidence XRD. In $\theta / 2 \theta$ geometry, as depicted in Figure 4.6, the sample holder rotates at a constant angular velocity, while the detector moves around the sample with twice that angular velocity. Therefore, the detector is always at a position of $2 \theta$ with respect to the incident beam. In the grazing incidence mode, the incident beam is kept at a constant small angle (e.g. $2^{\circ}$ ) to the sample surface. This mode is especially valuable for the characterization of thin films, because the incident beam always travels a longer way through the film and therefore the intensities in the final diffractogram are increased.

\subsection{Auger Electron Spectroscopy Depth Profiling}

A concentration depth profile can be obtained if the film is consistently sputtered and simultaneously a concentration analysis technique is applied. Auger Electron Spectroscopy Depth Profiling (AESDP) is based on this principle. While the film is

Figure 4.7: A KLL Auger process: a $\mathrm{K}$ electron of an atom in the sample is stricken out of its shell by collision with an electron from the source. An electron from the $\mathrm{L}$ shell falls into the vacancy in the $\mathrm{K}$ shell. The gained energy is directly transferred to another L electron, which therefore leaves the atom with a kinetic energy $E_{k i n}=E_{K}-E_{L_{1}}-E_{L_{2,3}}-\Phi_{A}$, where $E_{K}, E_{L_{1}}$ and $E_{L_{2,3}}$ are the energies of the $\mathrm{K}, \mathrm{L}_{1}$, and $\mathrm{L}_{2,3}$ shells and $\Phi_{A}$ is the

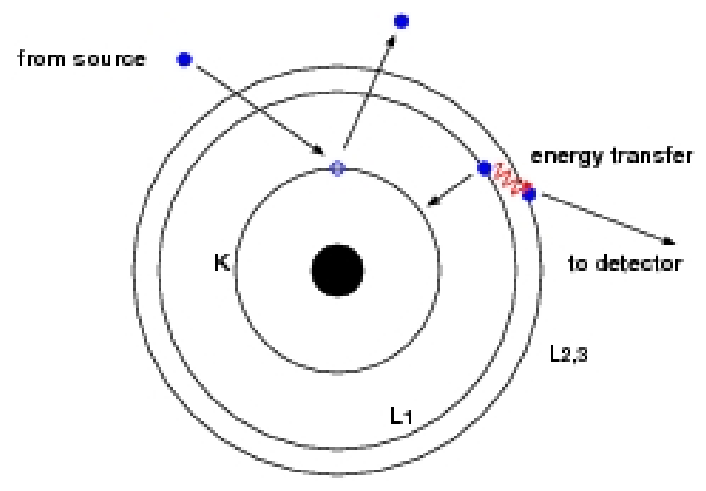
work function of the solid. 
being sputtered, the energy of Auger electrons created in the sample due to electronic excitation by an electron beam is being analyzed. As the energies of the Auger electrons are element specific and their line widths (i.e. peak-to-peak distances of the derived spectra) can be used to obtain the surface composition of the sample, the subsequent Auger electron spectra can be used to calculate a concentration depth profile. Figure 4.7 gives a schematic description of the Auger effect.

There are three limitations: first, the resolution is restricted by the information depth of the Auger spectra. Second, the sensitivity factors given in the literature vary strongly, and therefore the calculated concentration is very unreliable. Third, the sputtering process leads to a mixing of the film and may therefore weaken or destroy an originally existent concentration variation.

Several samples that showed a multilayer structure in TEM images were analyzed by AESDP. The measurements were performed by Ms. Wu using the VG Microlab 310D setup at the National Cheng Kung University of Tainan, Taiwan. 


\section{Chapter 5}

\section{Review of Previous Work}

This chapter reports on the results of two previous studies. First, in Section 5.1 , the work by Kröger and co-workers on the diffusion behaviour of metals in amorphous carbon is summarized [128]. In the following two sections, the findings for the first two systems, a-C:Cu and a-C:Ag, are described.

\subsection{Diffusion in Amorphous Carbon}

Kröger and co-workers examined the diffusion of metals as well as lighter elements and carbon in amorphous carbon. A $\delta$-layer of each element is introduced into an amorphous carbon film by either implantation or deposition of first an amorphous carbon sublayer, then a layer of the foreign element of about $1 \mathrm{~nm}$ width, and a subsequent amorphous carbon cover layer. The samples were annealed in a vacuum furnace at a pressure of $\sim 10^{-6}$ mbar for 10 min at temperatures up to $1200^{\circ} \mathrm{C}$.

Except for hydrogen/deuterium, neither dominantly $\mathrm{sp}^{3}$ nor dominantly $\mathrm{sp}^{2}$-bonded amorphous carbon allows diffusion up to temperatures of $900^{\circ} \mathrm{C}$ (carbon and nitrogen) or $1000^{\circ} \mathrm{C}$ (metals). Figure 5.1 shows the metal signals in RBS spectra at different annealing temperatures. It is quite evident that the line shape and intensity do not change for any of the three systems up to temperatures of $800^{\circ} \mathrm{C}$ for copper (middle panel in Figure 5.1) and $1000^{\circ} \mathrm{C}$ for tungsten and silver. The first remarkable feature is the additional peak in the RBS spectrum of the coppercontaining sample annealed at $1000^{\circ} \mathrm{C}$. This signal appears at the lower energy side to the otherwise unchanged copper signal and is generated by copper at greater depth. It is therefore concluded that this signal arises from copper that has diffused from the copper sample holder through the silicon substrate towards the substrate- 


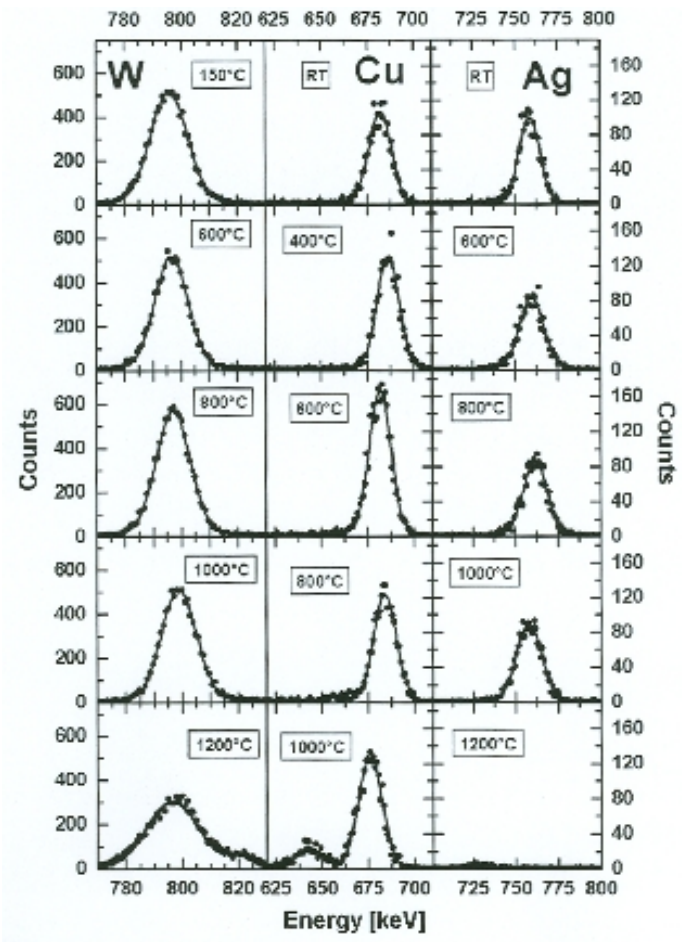

Figure 5.1: RBS analysis of the variation of the content and distribution of tungsten, copper, and silver in ta-C upon annealing (from [128]).

film interface. The diffusivity of copper in ta-C is still zero at this temperature: the copper from the sample holder does not enter the ta-C film and the deposited copper $\delta$-layer stays intact.

Only at $1200^{\circ} \mathrm{C}$, all three metals diffuse out of the sample; the distributions of metal atoms are broadened and the intensities significantly decrease (not shown for the copper case). At this temperature, amorphous carbon is converted into graphite.

It can be expected that the metal atoms within the films of this thesis will stay at rest, once its surroundings are no longer effected by incoming ions. The transport mechanism for metal as well as carbon atoms is purely driven by the ion-induced rearrangement processes described in Section 3.4 .

\subsection{Amorphous Carbon Containing Copper}

In previous studies, films grown by MSIBD of singly charged carbon and copper ions were analyzed [14, 147]. All these samples were grown onto p-type $\mathrm{Si}<100>$ substrates at room temperature using the MSIBD setup ADONIS. The ion energies and deposition temperature were kept at the same values for all samples, while the carbon/copper ion fluence ratio $r_{\text {fluence }}=$ carbon ion fluence/metal ion fluence was varied between 99 and 1. By this means, sputtering effects as well as the dependency 
Table 5.1: Deposition parameters of the a-C:Cu samples. All samples were deposited at room temperature onto $\mathrm{p}-\mathrm{Si}<100>$ substrates.

\begin{tabular}{ccccc}
\hline \hline Sample No. & $r_{\text {fluence }}$ & $E_{\text {ion }}\left(\mathrm{C}^{+}\right)[\mathrm{eV}]$ & $E_{\text {ion }}\left(\mathrm{Cu}^{+}\right)[\mathrm{eV}]$ & Deposited charge $[\mathrm{C}]$ \\
\hline 619 & 99 & 80 & 40 & 0.097 \\
$571 / 610$ & 19 & 80 & 40 & $0.065 / 0.1$ \\
568 & 9 & 80 & 40 & 0.05 \\
573 & 5.67 & 80 & 40 & 0.077 \\
$581 / 569$ & 4 & 80 & 40 & $0.095 / 0.12$ \\
574 & 2.33 & 80 & 40 & 0.041 \\
540 & 1.5 & 80 & 40 & 0.1 \\
513 & 1 & 80 & 40 & 0.11 \\
\hline \hline
\end{tabular}

of the cluster size and the quality of the a-C matrix on the metal content were analyzed. The deposition parameters are listed in Table 5.1 .

The a-C:Cu samples consist of nanometre size copper clusters distributed rather uniformly throughout an amorphous carbon matrix. The TEM micrograph of Figure 5.2 shows a sample deposited at a carbon/copper fluence ratio of $r_{\text {fluence }}=4$.

The RBS analysis shows that there is a moderate shortage of copper atoms per unit area compared to the areal density of deposited copper ions (see Figure 5.3). The loss has to be attributed to sputtering of copper surface atoms by impinging $\mathrm{C}^{+}$and $\mathrm{Cu}^{+}$ions. It is not possible to gain reliable information on the carbon areal density, because the carbon signal is superimposed on the signal of the silicon substrate. It is assumed that the sample compositions represent the $\mathrm{Cu}^{+}$ion fraction due to some sputtering of carbon atoms off the film surface. The analysis of the mean cluster size of each sample was conducted by different methods, depending on the possibilities the sample offered. The two samples with the highest copper content (samples 540 and 513, deposited at carbon/copper fluence ratios $r_{\text {fluence }}=1.5$ and 1) were analyzed by XRD. The mean cluster size can be calculated from the Lorentzian line width using the Scherrer formula given in Equation (4.5) [145]. However, the samples discussed here suffer from some compressive stress and the signal-to-noise ratio is weak due to the small absolute amount of copper. Therefore, a precise calculation is impossible and the results have to be regarded as an estimation of the mean cluster diameters of both films. For films containing smaller amounts of copper, XRD analysis is impossible. The cluster diameters of films 610 and 581 ( $r_{\text {fluence }}=$ 19 and 4, respectively) were determined from TEM micrographs and samples 568 and 574 ( $r_{\text {fluence }}=9$ and 2.33, respectively) were analyzed by D. Babonneau using 


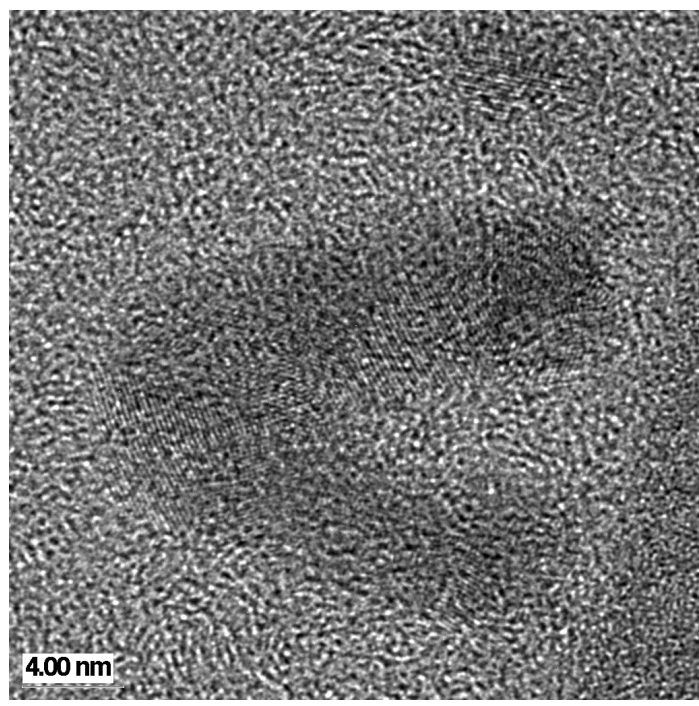

Figure 5.2: HRTEM of the a-C:Cu sample $581\left(r_{\text {fluence }}=4\right)$. Copper clusters are distributed uniformly across the film (from [14]).

Grazing Incidence Small Angle X-Ray Scattering (GISAXS). The results yielded by means of all three techniques are summarized in Figure 5.4. There is an obvious correlation between the copper ion fraction and the cluster size.

The structure of the amorphous carbon matrix was analyzed using Raman spectroscopy. Figure 5.5 shows the spectra corresponding to the different carbon/copper ion fluence ratios. At about $1350 \mathrm{~cm}^{-1}$ the intensity of the D-band rises with the copper content of the films. The matrix can be regarded as consisting of ta- $\mathrm{C}$ up to $r_{\text {fluence }}=19$ ( $\sim 5$ at.\% copper $)$.

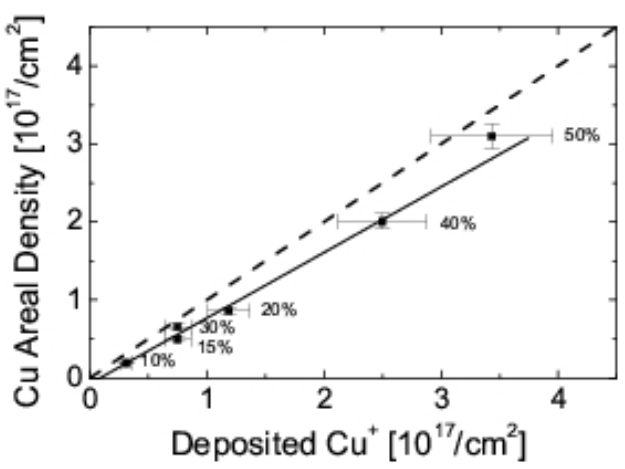

Figure 5.3: Results of the RBS analysis of the copper areal density in dependence on the deposited $\mathrm{Cu}^{+}$ion fluence (from [14]).

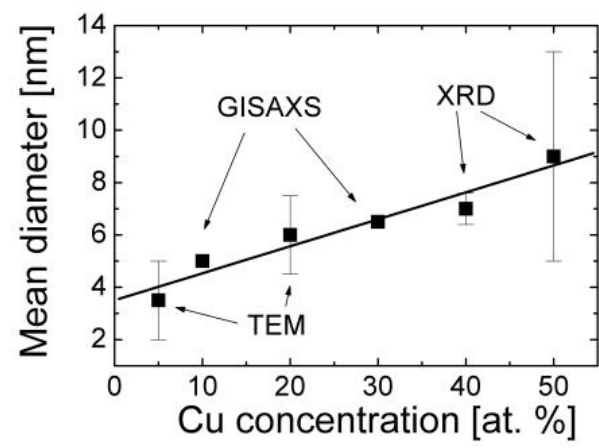

Figure 5.4: Results of the cluster size analysis using TEM and X-ray Diffraction methods of the copper-containing films grown with constant ion energy. The solid line is to guide the eye. 
Figure 5.5: Raman spectra of a-C: $\mathrm{Cu}$ films. The number next to each spectrum denotes the respective ion fluence ratio. The solid line shows the fit using a BWFfunction centred at about $1550 \mathrm{~cm}^{-1}$ and a Lorentzian at about $1350 \mathrm{~cm}^{-1}$. The sharp peak rising out of the G-band at $1550 \mathrm{~cm}^{-1}$ is due to atmospheric oxygen (from [14]).

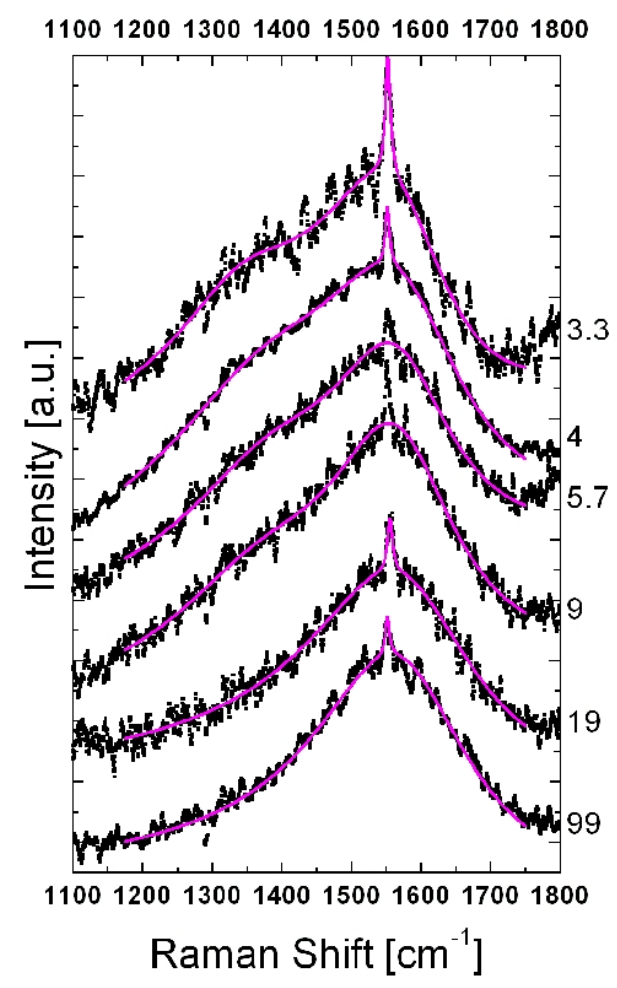

\subsection{Amorphous Carbon Containing Silver}

In a further experiment, samples were grown by co-deposition of singly charged ${ }^{12} \mathrm{C}^{+}$and ${ }^{107 / 109} \mathrm{Ag}^{+}$ions onto p-type $\mathrm{Si}<100>$ substrates using the MSIBD setup Adonis [148]. The deposition parameters are listed in Table 5.2. Transmission electron micrographs as well as EDX line scans of various samples show that the films contain hardly any silver except for a small amount at the interface between the substrate and the film, where at the beginning of the deposition process a mixture layer of silver-, carbon-, and silicon-atoms is formed. Only the TEM and EDX

Table 5.2: Deposition parameters of the a-C:Ag samples. All samples were deposited at room temperature onto $\mathrm{p}-\mathrm{Si}<100>$ substrates.

\begin{tabular}{ccccc}
\hline \hline Sample No. & $r_{\text {fluence }}$ & $E_{\text {ion }}\left(\mathrm{C}^{+}\right)[\mathrm{eV}]$ & $E_{\text {ion }}\left(\mathrm{Ag}^{+}\right)[\mathrm{eV}]$ & Deposited charge $[\mathrm{C}]$ \\
\hline 616 & 99 & 80 & 60 & 0.102 \\
620 & 19 & 80 & 60 & 0.094 \\
624 & 9 & 80 & 60 & 0.039 \\
$510 / 650$ & 4 & 80 & 60 & $0.07 / 0.071$ \\
649 & 2.33 & 80 & 60 & 0.069 \\
\hline \hline
\end{tabular}




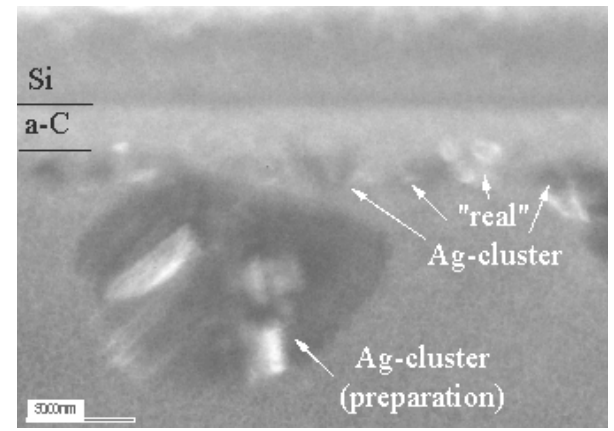

Figure 5.6: Dark field image of the a-C:Ag sample $650\left(r_{f}\right.$ luence $\left.=4\right)$. A number of small and large clusters are visible on the film surface. The large cluster was formed due to mechanical handling of the sample during TEM specimen preparation.

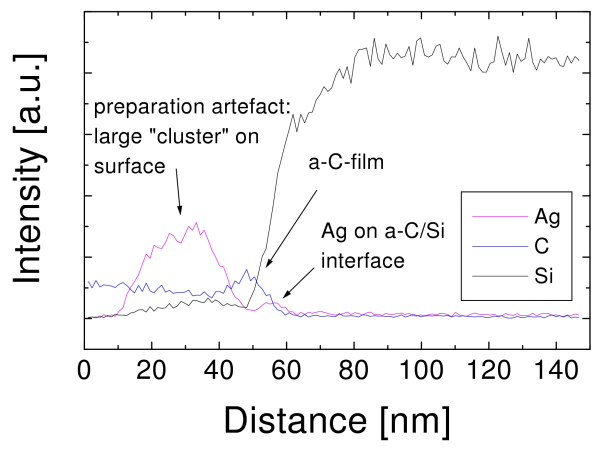

Figure 5.7: EDX-Linescan across sample 650. The silver signal has a small peak at the substrate-film interface and a large peak where the scan crosses a large silver cluster at the film surface.
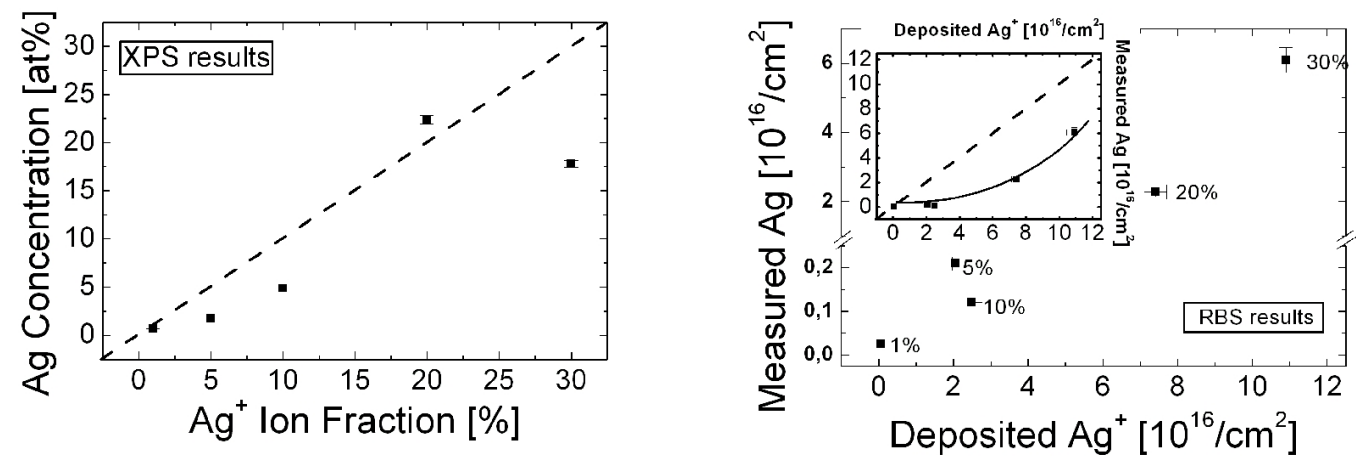

Figure 5.8: The composition analyses of the a-C:Ag films by XPS and RBS yield different results: XPS shows only small silver losses when regarding the surfaces of the films, RBS reveals a significant silver shortage in the integral film composition. For the RBS results, see also Reference [148]. 
analyses of sample 650 reveal a surface layer of silver clusters on top of a silverdepleted amorphous carbon film (see Figures 5.6 and 5.7).

The composition analyses by XPS and RBS yield very different results: while the surface-sensitive method XPS shows that the surface of the films has a slightly smaller silver concentration as compared to the ion fraction, the result of the RBS analyses is a significant shortage of silver in the integral film composition (see Figure 5.8). This phenomenon has two reasons: first, silver obviously segregates at the film surface, as verified by the TEM and EDX analyses of sample 650 depicted in Figures 5.6 and 5.7. Second, the silver is not tightly bonded to the amorphous carbon film surface and may easily be wiped off. The XPS measurement was conducted directly after deposition without breaking the vacuum. Therefore, the sample surfaces are not subject to any disturbance prior to the XPS measurements. When the samples are taken out of the vacuum chamber and then mounted onto the RBS sample holder, some or even all of the silver at the surface may accidentally be removed. In the TEM image of Figure 5.6, there are a number of very large clusters in addition to the layer of small clusters on the sample surface. These large clusters were most likely formed during the TEM specimen preparation, when the sample pieces were cleaned prior to being sandwiched. This also accounts for the complete loss of silver on the other films. Furthermore, if the silver is not incorporated into the film but segregates at the surface, there is a greater loss due to sputtering and the integral silver concentration is reduced compared to the silver ion fraction during deposition. 


\section{Chapter 6}

\section{Spontaneous Formation of Multilayers Reported in the Literature}

There are three other groups studying the self-organized formation of multilayers. $\mathrm{Wu}$ and Ting as well as Corbella and co-workers also analyzed metal-containing amorphous carbon [1, 2], whereas He and co-workers observed a similar self-organization effect for the immiscible system AuNi [3]. All of them use deposition techniques involving a film bombardment with hyperthermal species. In the following, their experimental results and model approaches are described.

\subsection{Experiments by $\mathrm{Wu}$ and Ting}

\subsubsection{Experimental Results}

Wu and Ting report on self-assembled alternating nanoscale layers of carbon and various metals [1]. They apply DC R-MSD for the deposition of their films. The deposition parameters can be summed up as follows: films were grown by sputtering from metal targets (nickel, copper, and platinum). The $\mathrm{Ar} / \mathrm{CH}_{4}$ gas ratios were $1 / 1$ and $3 / 1$. The pressure during deposition was $1.33 \times 10^{-2}$ mbar, the DC power was $100 \mathrm{~W}$, and the electrode distance was $4 \mathrm{~cm}$. At these deposition parameters, multilayer structures were found for all three metals except for the a-C:Ni film deposited at an $\mathrm{Ar} / \mathrm{CH}_{4}$ ratio of $1 / 1$. The multilayer structure emerges, however, when the $\mathrm{Ar} / \mathrm{CH}_{4}$ ratio is raised to $3 / 1$ (see Figure 6.1). In general, Wu and Ting found that the multilayers are more pronounced at the higher $\mathrm{Ar} / \mathrm{CH}_{4}$ ratio and the periodicities rise from $\infty, 13 \mathrm{~nm}$, and $10 \mathrm{~nm}$ up to $15 \mathrm{~nm}, 15 \mathrm{~nm}$, and $22 \mathrm{~nm}$ 


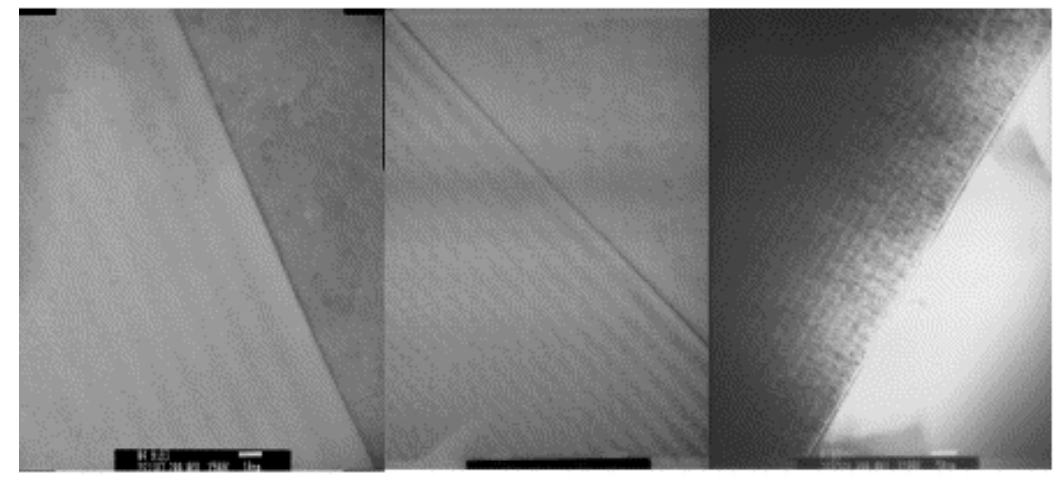

(a)

(b)

(c)

Figure 6.1: Multilayer structure of (a) a-C:Ni, (b) a-C:Cu, and (c) a-C:Pt. The Ar/ $\mathrm{CH}_{4}$ ratio during deposition was $3 / 1$ (from [1]).

for a-C:Ni, a-C:Cu, and a-C:Pt, respectively. An AES depth profile confirms the alternating concentration of carbon and platinum for one of the films.

\subsubsection{Catalysis Model for Multilayer Formation}

According to Wu's and Ting's model, the appearance of a periodical variation of the metal concentration of their magnetron-sputtered films is generated by two competing mechanisms: the difference in the deposition rates of carbon and the respective metal, and a catalytic behaviour of the metal. The mechanism is schematically shown in Figure 6.2. In a previous study, Wu and Ting had found that the deposition rate of all three metals are higher than that of carbon in reactive sputter deposition. Therefore, at the beginning of the film growth, a metal-rich layer containing a relative smaller amount of carbon should arise right at the interface to the substrate. This is in agreement with Wu's and Ting's experimental findigs. As known from the formation of carbon nanotubes, metals can act as catalysts for an enhanced growth of carbon. Wu and Ting conclude that this is the case also for their films, and therefore a carbon-rich layer containing a smaller amount of metal is deposited. As this new layer grows in thickness, it begins to shield the metal layer underneath and the carbon deposition rate decreases to a value smaller than the deposition rate of the metal. Again, a metal-rich layer is formed and so forth. 


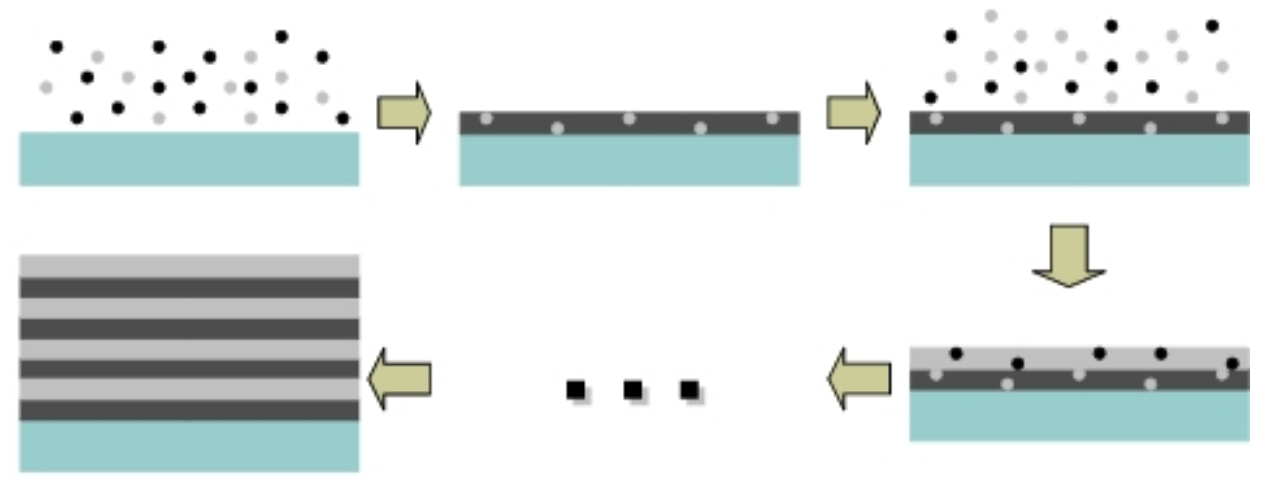

Figure 6.2: Multilayer formation model of $\mathrm{Wu}$ and Ting: in the beginning, metal deposition is favoured until carbon is preferentially deposited due to catalytic promotion of carbon deposition. The deposited carbon shields this catalytic behaviour and again a metal-rich layer is deposited. The diagram was generously provided by Ms. Wu [149].

\subsection{Experiments by Corbella and Co-workers}

Corbella and co-workers have been studying the incorporation of metals into amorphous carbon in order to enhance the ductility of their diamond-like carbon films. In their earlier studies, they deliberately grew metal-carbon multilayer films by alternate sputtering from two targets and found a decrease in the internal stress and therefore an improvement of the film adherence with respect to pure a-C films. However, the film hardness was not investigated [52]. Recent TEM studies on R-MSD films revealed a multilayer structure that evolved spontaneously during the deposition of films that were previously thought to have a homogeneous cluster distribution structure [2, 53, 54].

\subsubsection{Experimental Results}

The films were grown by reactive magnetron sputtering of either titanium, molybdenum, tungsten, or niobium targets using various $\mathrm{CH}_{4}$ gas flow fractions of up to $25 \%$ in an $\mathrm{Ar} / \mathrm{CH}_{4}$ mixture. The magnetron was driven by an asymmetric bipolar pulsed DC power supply with a pulse frequency of $10 \mathrm{kHz}$ and a power of $100 \mathrm{~W}$. The substrates were placed on a water-cooled sample holder and RF biased to $-200 \mathrm{~V}$. The TEM micrographs of samples containing 60, 25, and 5 at.\% titanium clearly show a nearly periodical contrast variation (see Figure 6.3). In all three cases, the layer 


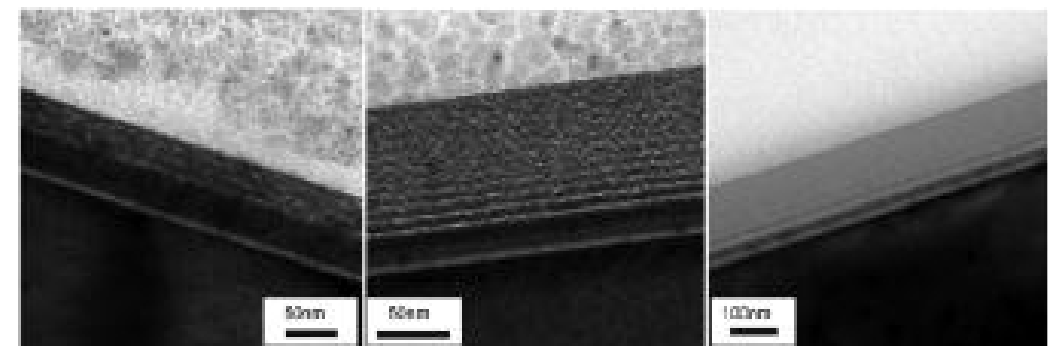

(a)

(b)

(c)

Figure 6.3: Multilayer structure of a-C:Ti films containing (a) 60 at.\% Ti, (b) 25 at.\% $\mathrm{Ti}$, and (c) 5 at.\% Ti (from [2]).

closest to the substrate is the thickest one. From electron energy loss spectroscopy mapping, it is concluded that the dark layers are titanium-enriched, whereas the brighter layers are rich in carbon. Selected area electron diffraction indicates that $\mathrm{TiC}$ crystallites are embedded in the darker layers. This is in agreement with the XRD results presented in an earlier publication [54]. The sample containing only 5 at.\% titanium is decomposed into two layers: a carbon layer on top of a titaniumrich interlayer adjacent to the substrate. In the other cases, the multilayer structure is lost towards the sample surface.

Further TEM analyses performed on films containing molybdenum and tungsten do not reveal any multilayers. While films with 85 at.\% and 50 at.\% of tungsten show a columnar structure inherited from pure tungsten films and a completely amorphous structure, respectively, films containing 60, 40 and 30 at.\% molybdenum are of a granular structure and SAED patterns as well as XRD indicate the presence of nanocrystalline MoC clusters.

\subsubsection{Cahn-Hillard Based Spinodal Decomposition Model}

Corbella and co-workers explain the multilayer formation in their experiments using a Cahn-Hillard based spinodal decomposition model, further developed by Puri and Binder [150]. They assume diffusion to be strongly enhanced in a region close to the surface due to the energy transfer from impinging atoms to the growing film. This favours the decomposition of the immiscible carbon and metal carbide phases. The determining factor for the concentration variation is the affinity of one of the components to the substrate. This leads to an enrichment of this component in a 


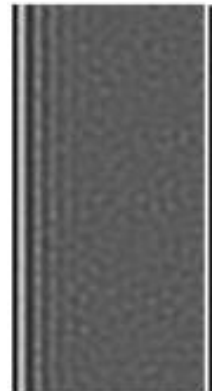

(a)

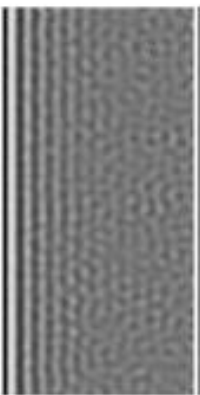

(b)

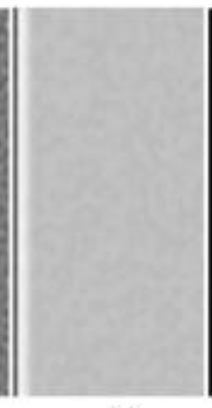

(c)

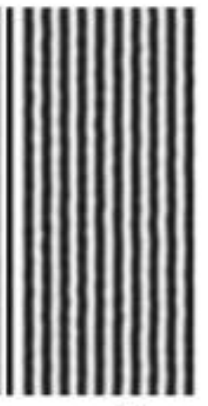

(d)

Figure 6.4: Simulation of Ti-C layer formation based on the CahnHillard Model. The assumed concentrations are (a) $u_{0}=-0.2$, (b) $u_{0}=0$, and (c) $u_{0}=0.4$ at a growth velocity of $v_{f g}=0.6$, and (d) $u_{0}=-0.2$ at a slower growth velocity of $v_{f g}=0.3$ (from [2]).

subsurface region and a depletion of the adjacent layer towards the surface. Spinodal decomposition then drives the formation of multilayers towards the film surface. The affinity of one of the components to the substrate is introduced into the chemical potential of the Cahn-Hillard model by a potential in the $z$-direction normal to the growth direction: $V(z)=h_{1}$ for $z<z_{0}$ and $V(z)=h_{1} z^{2} / z_{0}^{2}$ for $z>z_{0}$. The simulations were done using a new variable defined from the integral carbon concentration $c$ and the carbon concentrations $c_{\alpha}$ and $c_{\beta}$ in the carbon-deficient and carbon-rich areas: $u=\left(2 c-c_{\alpha}-c_{\beta}\right) /\left(c_{\alpha}-c_{\beta}\right)$. The ongoing growth of the film is taken into account by assuming a growth velocity $v_{f g}$. The wavelength of the segregation pattern $\lambda_{s p}$ is then determined by the ratio of the curvature of the free energy at the given initial concentration and the interfacial gradient coefficient. Figure 6.4 shows the results of the simulation with various concentrations and growth velocities. From these simulations, Corbella and co-workers conclude that the segregation induced by the substrate is more effective for concentrations in the middle of the miscibility gap (Figure 4(b)). A disturbance is generated by the growth velocity, as the time scale during which the enhanced diffusion process is active is too short. Well pronounced multilayers should therefore be found at smaller growth velocities $v_{f g}$ (Figure $4(\mathrm{~d})$ ).

\subsection{Experiments by $\mathrm{He}$ and Co-workers}

The experiments by He and co-workers show that the spontaneous formation of alternating layers is not limited to metal-carbon systems. They implement an ion beam assisted evaporation setup (IBAD) for the growth of AuNi alloy films. Gold and nickel show very limited mutual solubility. 


\subsubsection{Experimental Results}

Films were grown at room temperature onto $\mathrm{Si}<100>$ substrates at deposition rates of $0.3 \AA / \mathrm{s}$ and $0.9 \AA / \mathrm{s}$ for gold and nickel, respectively. The assisting $\mathrm{Ar}^{+}$ ions were of energies between 100 and $800 \mathrm{eV}$ and beam current densities of 25 or $50 \mu \mathrm{A} / \mathrm{cm}^{2}$. The film morphology was analyzed by X-ray reflectivity (XRR) measurements. The spectra show a number of Bragg peaks indicating a multilayer structure (see Figure 6.5 (a)). A dependence of the multilayer period and amplitude on the ion energy as well as the ion flux is discernible. The variation of the periodicity is given in Figure 6.5 (b). The periods of the multilayers are smaller than what is found in the carbon-metal films investigated by $\mathrm{Wu}$ and Ting, Corbella and coworkers, and this work. The periodicities vary in the range between 2 and $8 \mathrm{~nm}$. They apparently rise monotonically with the ion energy at higher ion energies and ion fluxes, but a dent is obvious around $300-400 \mathrm{eV}$ at beam fluxes of $50 \mu \mathrm{A} / \mathrm{cm}^{2}$. It is especially noteworthy that no multilayer evolves when the film is grown without any ion beam assistance.

Figure 6.5: (a) XRR spectra of AuNi films grown at various assistance ion energies and ion fluxes. The Bragg peaks show variations of intensities and peak positions. (b) The multilayer periodicity as calculated from the spectra (from [3]).
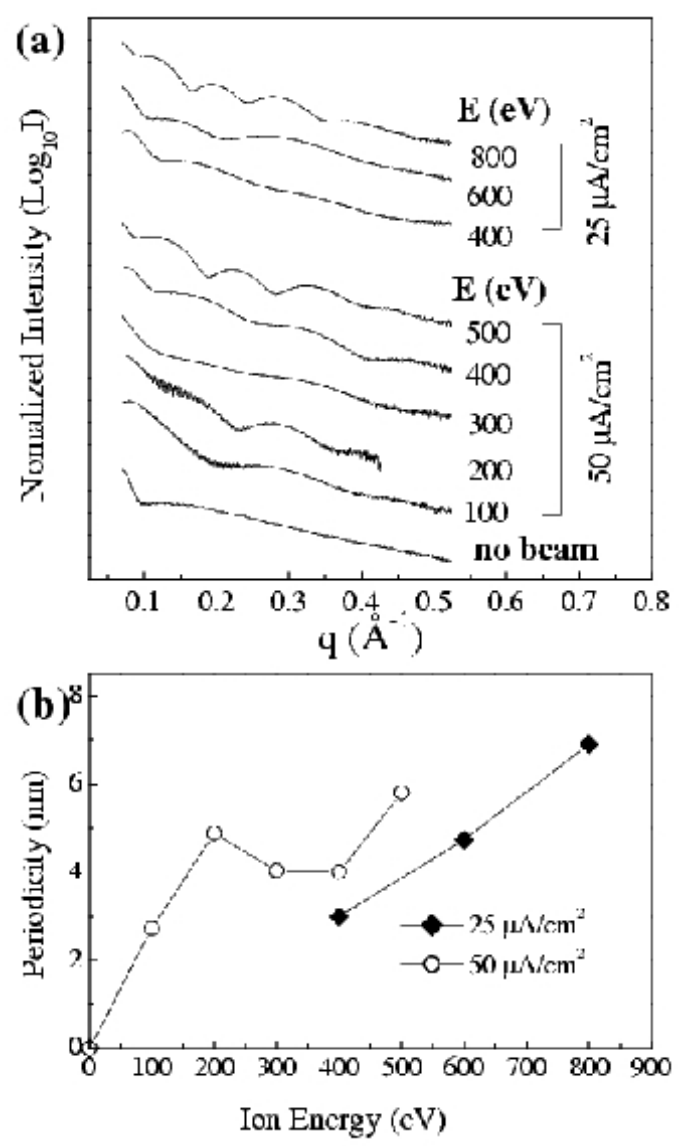


\subsubsection{Combined Cahn-Hillard/Ion Beam Induced Segregation Model}

He and co-workers present a model for the formation of multilayers in their IBAD process that is similar to the model presented by Corbella and co-workers (see Section 6.2.2. However, they extend the potential $V(z)$ by a radiation-induced term. They assume two competing transport mechanisms within a subsurface region of nanometre depth: one is provided by spinodal decomposition in the immiscible AuNi alloy favouring a phase separation and therefore resulting in a mass flow $J_{A}^{\text {chem }}$ for species $\mathrm{A}$ in the direction of the concentration gradient. The other is the bombardmentinduced segregation in growth direction yielding a mass flow $J_{A}^{B I S}$ for species A. At the early stages of growth, the bombardment-induced segregation leads to a depletion of one element (say A) at the surface and an enrichment in the subsurface region. The spinodal decomposition flux $J_{A}^{\text {chem }}$ is therefore adjusted in the same direction as $J_{A}^{B I S}$ and the surface depletion of $\mathrm{A}$ is enhanced. Because the concentration is determined by the deposition flux ratio before diffusion, the surface concentration is higher than in the just buried surface layer, as the film grows. Therefore, $J_{A}^{\text {chem }}$ now acts in the direction opposite $J_{A}^{B I S}$, the over-all mass transport of A being governed by the total flux $J_{A}^{\text {tot }}=J_{A}^{\text {chem }}+J_{A}^{B I S}$. If $J_{A}^{B I S}$ is dominant, the A-atoms from the newly deposited surface are still being transported into the A-rich subsurface region. The A-concentration profile in this zone becomes broader and grows into the depleted layer until A reaches a critical concentration. Below this critical concentration, $J_{A}^{\text {chem }}$ becomes dominant in $J_{A}^{\text {tot }}$ and A enriches the surface. This feedback loop

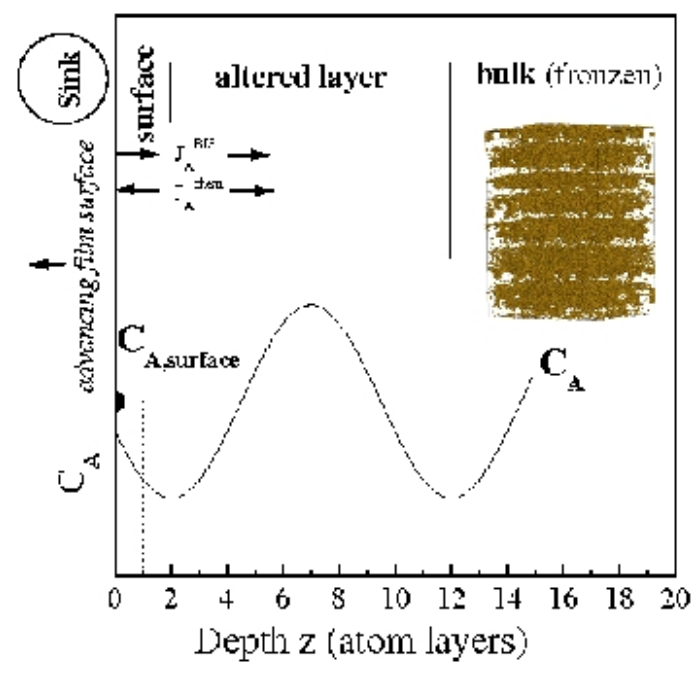

Figure 6.6: Schematic illustration showing the concentration profile for species A and the two fluxes $J_{A}^{\text {chem }}$ and $J_{A}^{B I S}$ driven by spinodal decomposition and bombardment-induced segregation, respectively. The inset shows a kinetic Monte Carlo simulation with $\beta J=-0.45$ and $\beta U=0.3$, the quantity $\beta$ is not defined in the publication (from [3]). 
finally results in the formation of multilayers. Figure 6.6 schematically demonstrates this scenario.

He and co-workers applied an ABV ${ }^{1}$ model in order to perform Monte Carlo simulations. For the sake of simplicity, only the interplay of equilibrium segregation and phase separation in an advancing nanoscale subsurface diffusion zone are considered. This assumption represents the growth of AuNi films under low-energy ion irradiation. The system Hamiltonian is given by $H=H_{0}+J \sum_{<i, j>} \sigma_{i} \sigma_{j}+U \sum_{<i, j>}\left(\sigma_{i}^{2} \sigma_{j}+\right.$ $\left.\sigma_{i} \sigma_{j}^{2}\right)+K \sum_{<i, j>} \sigma_{i}^{2} \sigma_{j}^{2}$, where the spin variable $\sigma_{1}=(1,-1,0)$ represents that site i is occupied by either an A-atom, a B-atom, or a vacancy, respectively. The segregation process is represented by the parameter $U \neq 0$, which is determined by the difference in surface energies of $\mathrm{A}$ and $\mathrm{B}$. The phase separation is given by the parameter $J<0$. The parameter $K$ is not specified in the publication. For the NiAu system, both processes are strong. Therefore, the calculation yielding the inset in Figure 6.6 was obtained by using strongly competing parameters. The resulting morphology resembles the multilayer structure of the films.

\footnotetext{
${ }^{1}$ The ABV model regards a binary alloy $\mathrm{AB}$ with pairwise interactions between nearest neighbours. Direct interchange of atoms is impossible. Transport can only be generated by vacancies [151.
} 


\section{Chapter 7}

\section{Morphology of MSIBD-Grown a-C:Me films}

Starting from the results summarized in Chapter 5, the following mass selective ion beam deposition (MSIBD) experiments were undertaken: First, the influence of the copper ion energy on both the development of clusters as well as the matrix quality was analyzed. These films were grown using the MSIBD setup ADONIS. Second and third, a-C:Au and a-C:Fe films were grown. In both cases, the variation of the metal/carbon ion fluence ratio was in the main focus. While the a-C:Fe films were also grown using ADONIs, the a-C:Au films were prepared with the second MSIBD setup Mr. STRInger. All films were grown on p-type silicon substrates in $<100>$ orientation. The substrates were pretreated by $1 \mathrm{keV} \mathrm{Ar}^{+}$sputtering in order to remove the native $\mathrm{SiO}_{2}$ layer and amorphize the substrate surface. In some cases, a pure amorphous carbon layer was deposited first, before the co-deposition process was initiated (see the respective tables of deposition parameters). This prevents the formation of a ternary mixture layer of silicon, carbon, and the metal at the interface. Some of the results presented in this chapter were also published in References [14] and [152].

\section{1 a-C:Cu Film Morphology Dependence on $\mathrm{Cu}^{+}$ Ion Energy}

A set of films was grown for each of the two carbon/copper ion fluence ratios of 4 and 19 at copper energies varying from 40 to $80 \mathrm{eV}$. The deposition parameters are summarized in Table 7.1. From the RBS spectra, a strong dependency of the incor- 
Table 7.1: Deposition parameters of the second set of a-C:Cu samples. For the two ion fluence ratios 4 and 19 a set of samples were grown with various $\mathrm{Cu}^{+}$ion energies. All samples were deposited at room temperature onto $\mathrm{p}-\mathrm{Si}<100>$ substrates.

\begin{tabular}{ccccc}
\hline \hline Sample No. & $r_{\text {fluence }}$ & $E_{\text {ion }}\left(\mathrm{C}^{+}\right)[\mathrm{eV}]$ & $E_{\text {ion }}\left(\mathrm{Cu}^{+}\right)[\mathrm{eV}]$ & Deposited charge $[\mathrm{C}]$ \\
\hline 715 & 19 & 100 & 40 & 0.078 on 0.02 ta-C \\
717 & 4 & 100 & 40 & 0.089 on 0.02 ta-C \\
732 & 19 & 100 & 60 & 0.1 on 0.02 ta-C \\
727 & 4 & 100 & 60 & 0.1 on 0.02 ta-C \\
729 & 19 & 100 & 80 & 0.18 on 0.02 ta-C \\
724 & 19 & 100 & 80 & 0.06 on 0.02 ta-C \\
737 & 4 & 100 & 80 & 0.167 on 0.02 ta-C \\
\hline \hline
\end{tabular}

porated amount of copper on the copper ion energy is obtained, as shown in Figure 7.1. Sputtering becomes stronger at higher copper ion energies, but in particular the higher carbon ion energy leads to far more aggressive copper sputtering (see Table 7.2). The values of the sputtering losses obtained from RBS measurements and the sputtering losses expected from the SRIM simulation, taking into account the carbon ion fluence ratio as well as a surface composition according to the ion fluence ratio, are partly in the same range. However, the experimental values are generally slightly higher with a few strong deviations. It has to be concluded that some of the deposited copper atoms segregate at the surface and therefore the sputtering of copper atoms is enhanced.

The carbon matrices of the samples were analyzed by Raman spectroscopy. The spectra are shown in Figure 7.2. All samples exhibit a typical broad skew signal centred at about $1580 \mathrm{~cm}^{-1}$ (G-band) with a more or less pronounced shoulder at about $1350 \mathrm{~cm}^{-1}$ (D-band). The sharp peak superimposing the G-band is generated by atmospheric oxygen. The spectra were fit using the BWF function given in Equation (4.4) in order to account for the G-band and another two Lorentzian

Table 7.2: $\quad$ Sputtering yields $s_{C u}$ and $s_{C}$ of copper and carbon atoms, respectively, by impinging carbon and copper ions of various ion energies. Calculated using SRIM [112].

\begin{tabular}{cccc}
\hline \hline $\begin{array}{c}\text { Impinging } \\
\text { Ion }\end{array}$ & $\begin{array}{c}\text { Ion Energy } \\
{[\mathrm{eV}]}\end{array}$ & $s_{\mathrm{Cu}}$ & $s_{C}$ \\
\hline $\mathrm{C}$ & 80 & 0.25 & 0.05 \\
$\mathrm{C}$ & 100 & 0.40 & 0.07 \\
$\mathrm{Cu}$ & 40 & 0.1 & 0 \\
$\mathrm{Cu}$ & 60 & 0.23 & 0 \\
$\mathrm{Cu}$ & 80 & 0.33 & 0 \\
\hline \hline
\end{tabular}



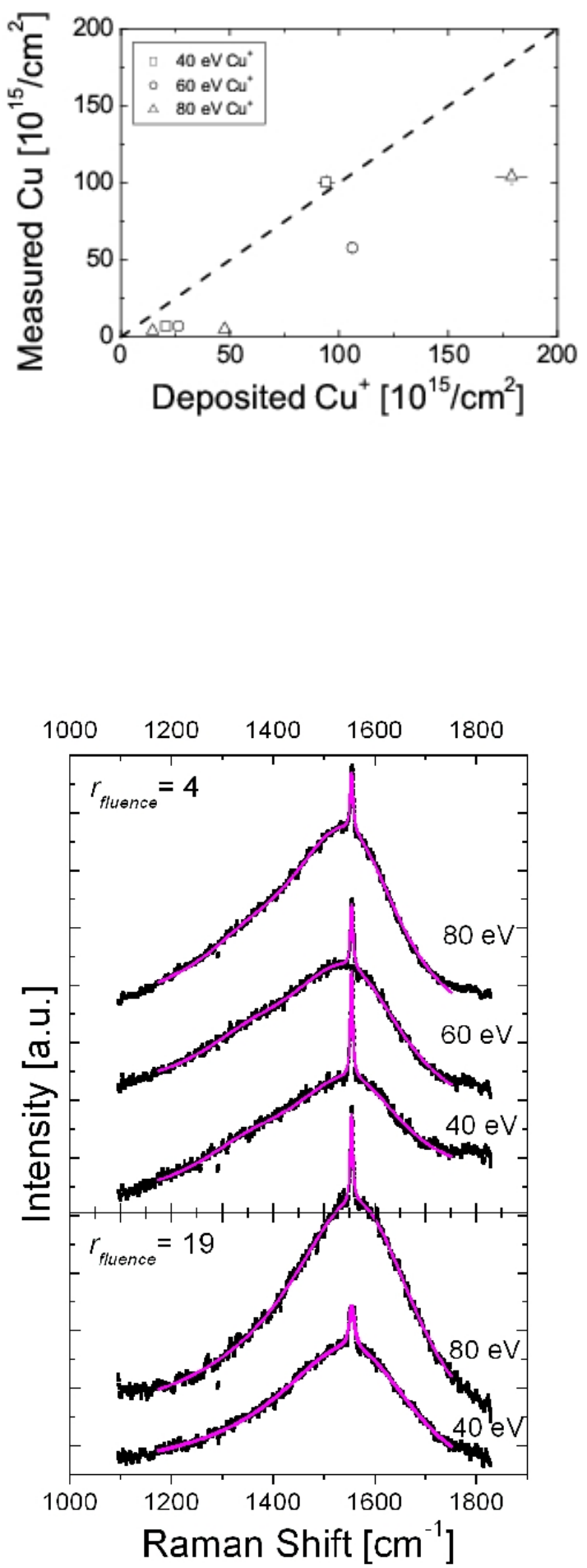

Figure 7.1: Composition of a-C:Cu samples deposited with $\mathrm{Cu}^{+}$ion energies of 40,60, and $80 \mathrm{eV}$ and carbon/copper ion ratios of 19 and 4 (from [14]).
Figure 7.2: Raman spectra of a$\mathrm{C}: \mathrm{Cu}$ samples deposited at $\mathrm{Cu}^{+}$ion energies of 40, 60 and $80 \mathrm{eV}$. The purple line represents the fit. There is apparently no dependence of the matrix structure on the $\mathrm{Cu}^{+}$ion energy (from [14]). 
Figure 7.3: HRTEM of a-C:Cu sample $717\left(E_{C u}=40 \mathrm{eV}\right)$. Crystalline copper clusters are distributed uniformly across the film (from [14]).

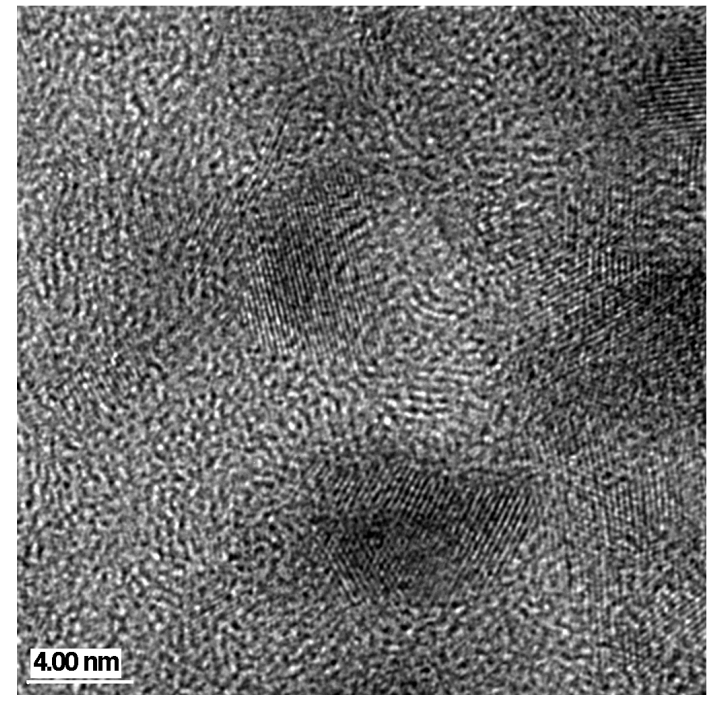

functions for the D-band and the oxygen signal. The samples obtain a slightly higher $\mathrm{sp}^{3}$-bonding content than the ones reported on in Section 5.2, as can be seen by the smaller intensity of the D-band. This is clearly a consequence of the deposition at the optimal carbon ion energy of $100 \mathrm{eV}$. However, the $\mathrm{sp}^{3}$-bonding content does not depend on the energy of the $\mathrm{Cu}^{+}$ions, as the intensity ratio of the G-band and D-band remains constant. The dependency on the deposited copper ion fraction is equivalent to what has been discovered on the first set of samples: smaller copper ion fractions result in higher $\mathrm{sp}^{3}$-bonding contents. The matrix structure evidently depends on the deposited $\mathrm{Cu}^{+}$ion fraction and not primarily on the actually incorporated copper concentration.

The only sample of this set that showed crystalline areas in TEM micrographs is sample 717, deposited at a copper ion energy of $40 \mathrm{eV}$ and a carbon/copper ion fluence ratio of 4 (see Figure 7.3). For all the other samples, the copper concentration is too low for the formation of clusters that are large enough to be distinguishable in TEM. The mean particle diameter of sample 717 is about $5 \mathrm{~nm}$. This is very close to the mean particle size of $6 \mathrm{~nm}$ of the equivalent sample deposited with the same parameters but $80 \mathrm{eV} \mathrm{C}^{+}$ion energy. From this, it is concluded that the carbon ion energy has no significant influence on the cluster diameter.

\subsection{Amorphous Carbon Containing Gold}

As gold or $\mathrm{AuCl}$ yield very poor ion currents from a Sidenius type ion source, the a-C:Au films were grown using the MSIBD setup Mr. STRINGER, where a Penning 
Table 7.3: Deposition parameters of the a-C:Au samples. All samples were deposited at room temperature onto $\mathrm{p}-\mathrm{Si}<100>$ substrates.

\begin{tabular}{|c|c|c|c|}
\hline Sample No. & $r_{\text {fluence }}$ & $E_{\text {ion }}\left(\mathrm{C}^{+}\right)$and $\left(\mathrm{Au}^{+}\right)[\mathrm{eV}]$ & Deposited charge $[\mathrm{C}]$ \\
\hline s008 & 39 & $\sim 100$ & 0.52 \\
\hline s006 & 19 & $\sim 100$ & 0.26 \\
\hline s010 & 9 & $\sim 100$ & 0.36 on 0.075 ta-C \\
\hline s033 & 9 & $\sim 100$ & 0.053 on 0.04 ta-C \\
\hline s031 & 5.67 & $\sim 100$ & 0.085 on 0.015 ta-C \\
\hline s007 & 4 & $\sim 100$ & 0.25 \\
\hline s009 & 4 & $\sim 100$ & 0.41 on 0.075 ta-C \\
\hline s034 & 4 & $\sim 100$ & 0.23 on 0.02 ta-C \\
\hline s035 & 4 & $\sim 200$ & 0.18 on 0.024 ta-C \\
\hline s032 & 3 & $\sim 100$ & 0.12 on 0.026 ta-C \\
\hline
\end{tabular}

sputter ion source is available (see Section 3.1.1). The deposition parameters are listed in Table 7.3 .

Figure 7.4 depicts an overview transmission electron micrograph of the entire cross-section of the a-C:Au sample s007, deposited at a carbon/gold fluence ratio of $r_{\text {fluence }}=4$. A multilayer structure is obvious in the overview micrograph with layer distances between 3 and $15 \mathrm{~nm}$. The HRTEM micrograph reveals a nanocrystalline structure in the dark layers and an amorphous structure in the brighter regions. Fourier transformations of the micrographs of the crystalline particles match the $d_{111}$ lattice plane spacing of gold of $2.35 \AA$. An X-ray diffractogram of this sample shows several gold signals. Using the Scherrer formula given in Equation (4.5) [145], an approximate average cluster diameter of $4.2 \mathrm{~nm}$ from the line width of the gold (111) signal can be derived.

The composition of the layers is verified by the EDX line scan shown in Figure 7.5: The first dark layer following the interface to the silicon substrate is a mixture of carbon, gold, and silicon atoms formed at the beginning of the deposition process when the first incoming ions were subplanted into the topmost nanometres of the silicon substrate. The successive layers are alternately Au-deficient and -rich. However, corresponding to the very broad bright layer following the mixture layer, there is a small peak in the gold signal, while no nanocrystals are visible in TEM images.

The chemical composition was also analyzed by AES depth profiling. The result is depicted in Figure 7.6. The concentration of gold, carbon, and silicon are given as a function of the scan time. Due to the ongoing sputtering of the film, the scan 

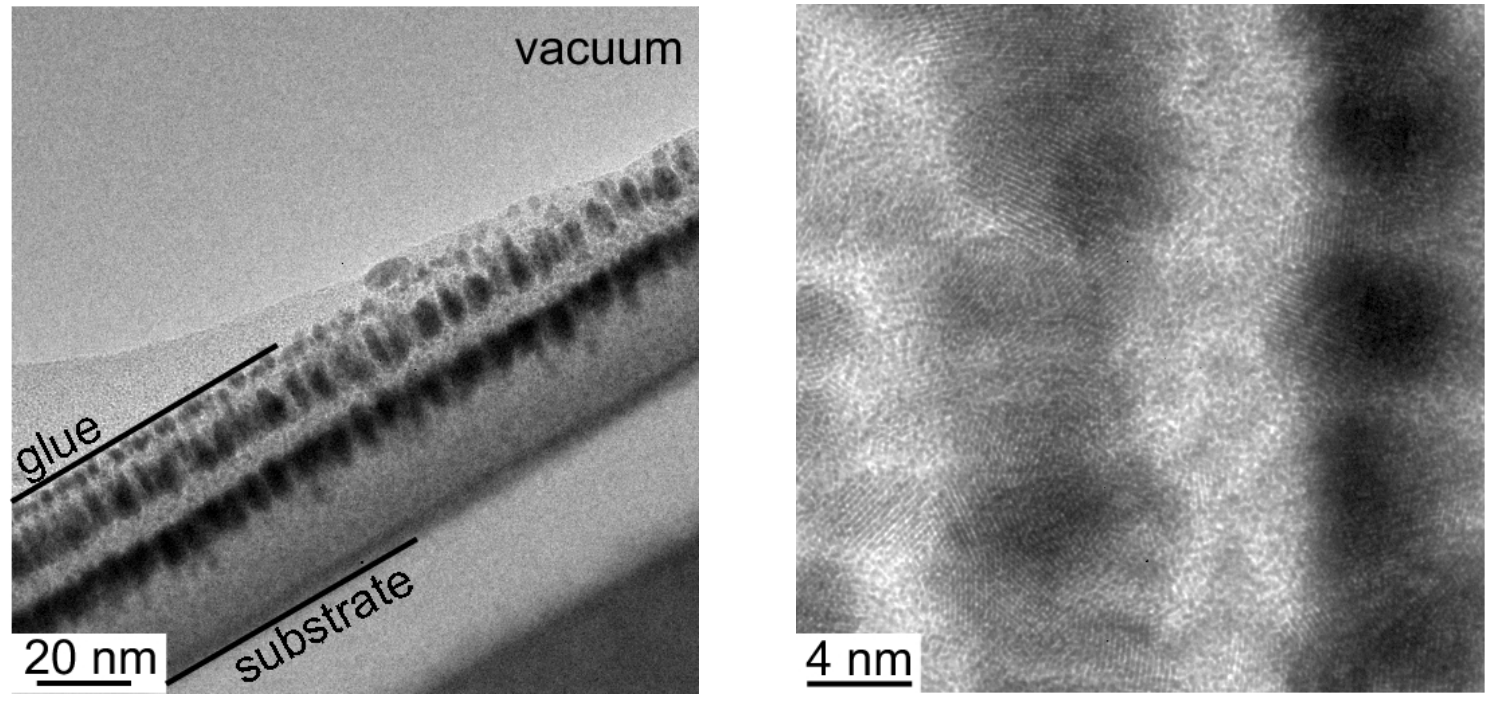

Figure 7.4: TEM micrographs of the a-C:Au sample s007 $\left(r_{\text {fluence }}=4\right)$. The layer structure is clearly visible in the overview micrograph (from [152]). The HRTEM image shows crystalline particles in the darker layers and an amorphous structure of the brighter layers.

Figure 7.5: EDX line scan across the a-C:Au sample s007. The topmost layer of small clusters is not visible in this line scan. The first two peaks in the gold signal arise from the second and third layer, the third signal cannot be identified from the TEM image. There is a small gold peak from the mixture layer near the film-substrate interface.

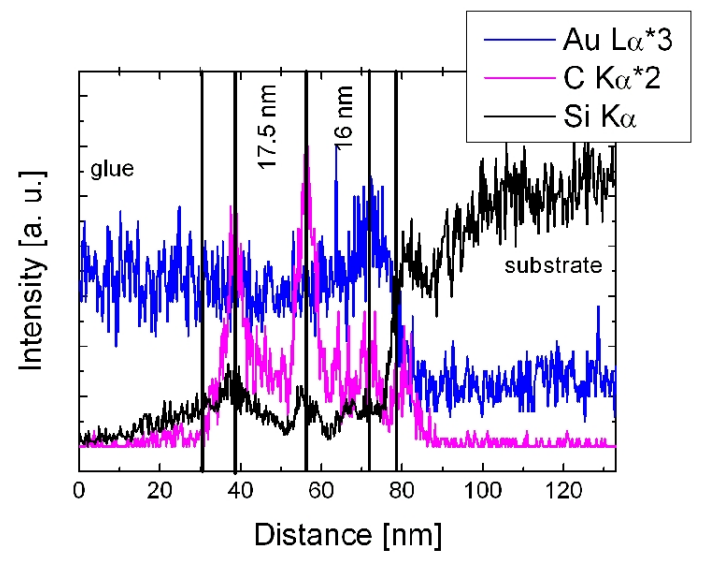



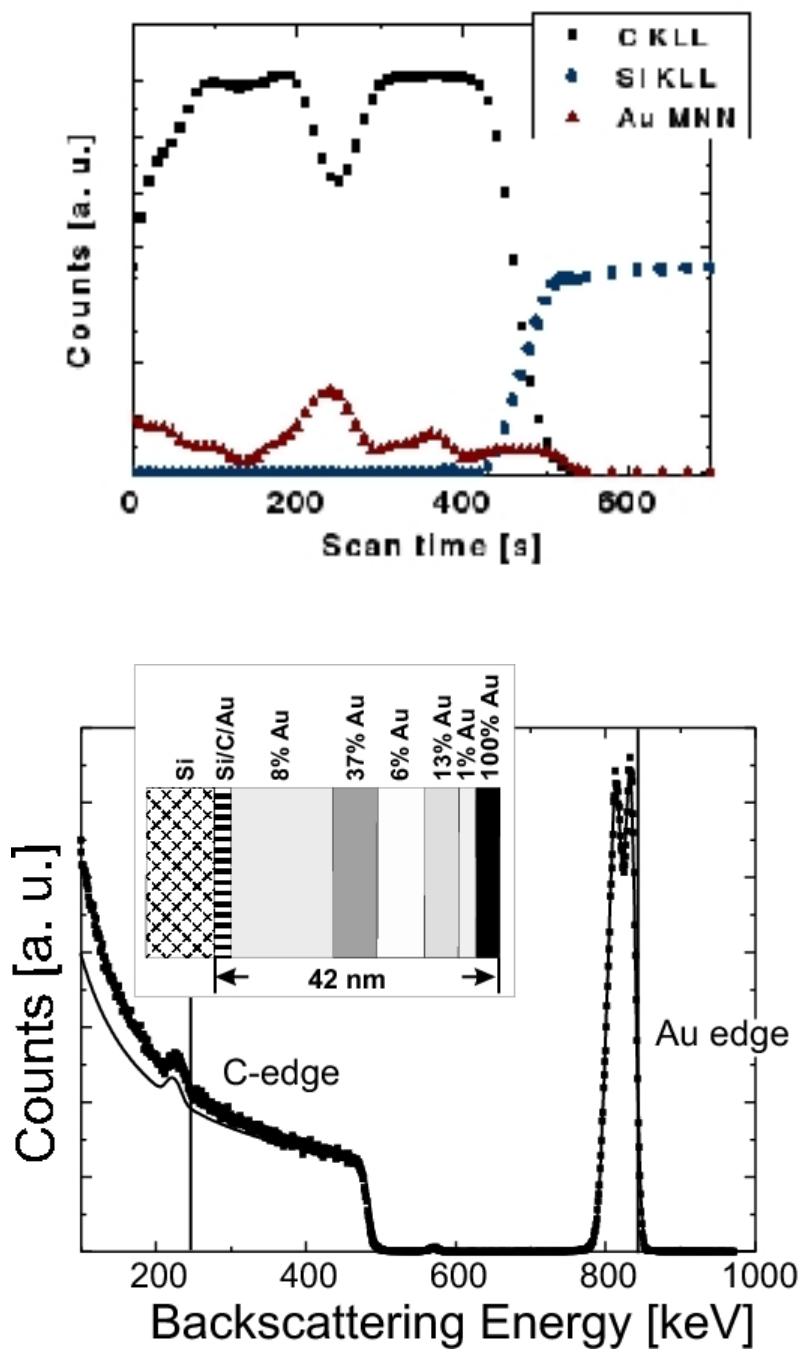

Figure 7.6: AES depth profile of the a-C:Au sample s007. The intensity variation of the gold signal correlates with the layers seen in the TEM micrographs but is not very pronounced.
Figure 7.7: RBS spectrum of the a-C:Au sample s007. The gold signal exhibits a double peak structure that indicates a multilayer morphology of the film. The inset shows the layer sequence that was assumed for the simulation of the spectrum. The layer thicknesses, starting from the surface, are $3.5 \mathrm{~nm}, 2.5 \mathrm{~nm}, 5 \mathrm{~nm}$, $7 \mathrm{~nm}, 6.5 \mathrm{~nm}$, and $15 \mathrm{~nm}$. The mixture layer has a thickness of $2.5 \mathrm{~nm}$ (from [152]).

time correlates with the depth of the film. Therefore, the spectrum can be read as a depth profile. The concentration variation derived from this correlates well with the EDX line scan and TEM results, however, the structure is not as pronounced. This sample was also the only one to show a variation of the gold concentration in AES depth profiling. As stated in Section 4.5, the multilayer structure of the film may be destroyed due to ion beam mixing in the process of sputtering the film for depth profiling.

Figure 7.7 shows the RBS spectrum of the same sample. The gold signal exhibits a double peak structure, which indicates an inhomogeneous gold concentration depth profile. Using the information gained from TEM for a RUMP analysis of the appendant RBS spectrum, one obtains the simulated plot depicted in Figure 7.7. The small 

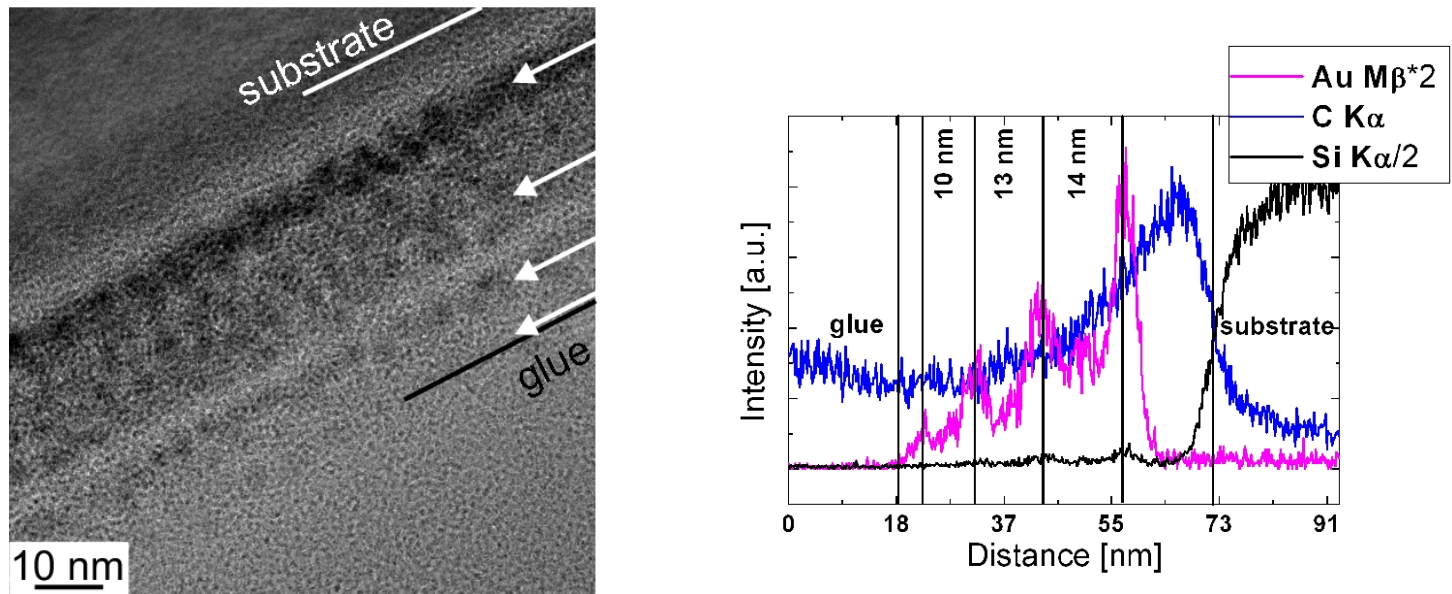

Figure 7.8: TEM overview micrograph of the a-C:Au sample s009 $\left(r_{\text {fluence }}=4\right)$. The deposition parameters were generally the same as for sample s007. The multilayer structure is not very pronounced in the micrograph but is evident from the EDX line scan (from [114]). The arrows in the TEM image indicate the positions of the maxima of the gold signal in the EDX line scan.

signal at $\sim 570 \mathrm{keV}$ arises from argon that is incorporated in the substrate due to the pre-deposition argon sputter cleaning process. The inset of Figure 7.7 accounts for the assumed layer composition for the RUMP simulation. The best fit results in the following composition: The first layer adjacent to the silicon/carbon/gold mixture layer at the substrate film interface contains 8 at.\% gold at a thickness of $15 \mathrm{~nm}$. The following layers contain 37 at.\%, 6 at.\%, 13 at.\%, 1 at \%, and 100 at.\% gold at thicknesses of $6.5 \mathrm{~nm}, 7 \mathrm{~nm}, 5 \mathrm{~nm}, 2.5 \mathrm{~nm}$, and $3.5 \mathrm{~nm}$, respectively. The topmost two gold-rich layers give rise to the higher energy peak of the spectrum, with the 6 at.\% layer generating the dent between the two peaks. The lower energy peak is dominated by the third gold-rich layer. The 8 at.\% gold layer and the mixture layer lead to the shoulder at the lower energy side of the signal.

Figure 7.8 shows an overview TEM micrograph and EDX line scan of sample s009. This sample was deposited principally under the same conditions as sample s007. The sample was nominally grown thicker and onto a pre-deposited amorphous carbon layer. However, the sample is only about half as thick as sample s007 and seems rather inhomogeneous. Therefore, some irregularities during deposition have to be presumed. Sample s009 does not show such a pronounced layer structure in the TEM micrograph as sample s007. Nevertheless, the variation of the gold 

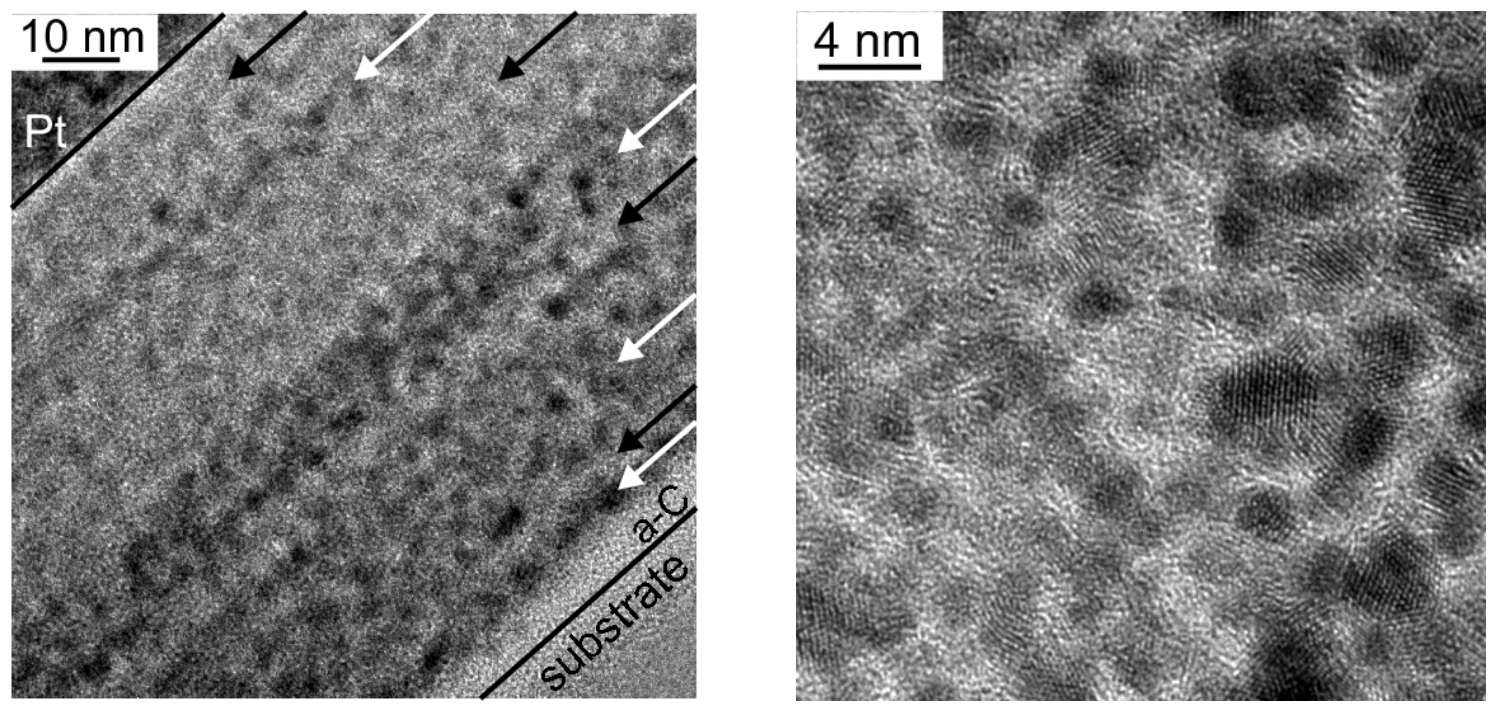

Figure 7.9: TEM micrograph of the a-C:Au sample s034 $\left(r_{\text {fluence }}=4\right)$. Layers of higher and lower cluster density are indicated by arrows in the overview micrograph. The HRTEM image was taken from the middle of the film. On the right of the HRTEM image a layer of greater cluster density can be seen, while on the left the cluster density is smaller. The image clearly shows the crystallinity of the particles.

concentration is clearly notable in the EDX line scan. The general decrease of the gold intensity is caused by the wedge shape generated in the ion mill thinning process.

Two further samples were grown at an ion fluence ratio of $r_{\text {fluence }}=4$. These two samples were prepared using a focused ion beam system for TEM analysis. In the corresponding overview micrographs, the sample surface is indicated by the interface to the platinum cover layer.

Sample s034 was another attempt to grow a thicker sample with a larger number of pronounced layers. The overview micrograph as well as a HRTEM image are shown in Figure 7.9. The sample is about $80 \mathrm{~nm}$ thick as compared to a thickness of about $60 \mathrm{~nm}$ of sample s007. However, the layer structure is by far not as pronounced and shows no clear periodicity. There are eight alternately darker and brighter layers distinguishable on top of an amorphous carbon layer on the substrate. The layers are indicated by arrows in the overview micrograph in Figure 7.9, The layer thicknesses are (starting from the substrate): $5.3 \mathrm{~nm}, 2.6 \mathrm{~nm}, 18.4 \mathrm{~nm}, 3.2 \mathrm{~nm}$, $12.1 \mathrm{~nm}, 20.0 \mathrm{~nm}, 3.7 \mathrm{~nm}$, and $15.8 \mathrm{~nm}$. The transition from the amorphized 


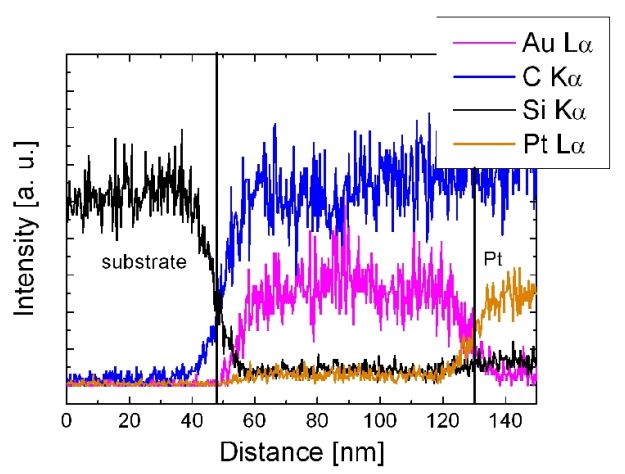

Figure 7.10: EDX line scan across the a$\mathrm{C}: \mathrm{Au}$ sample s034. A variation of the gold concentration is not clearly detectable. The carbon signal rises at the interface to the substrate due to the pre-deposited amorphous carbon layer and a possibly gold-depleted zone of the a-C:Au film.

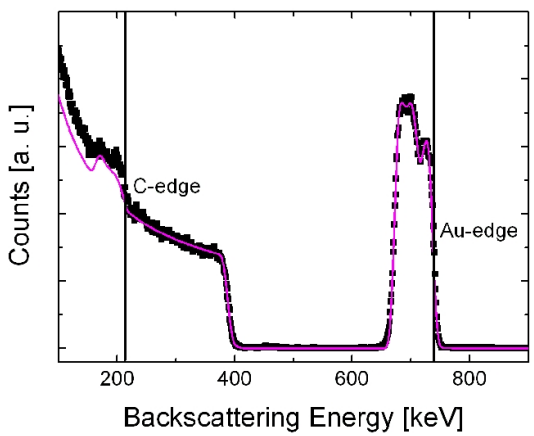

Figure 7.11: RBS spectrum of the a$\mathrm{C}: \mathrm{Au}$ sample s034. The gold signal shows a clear double peak structure. A small dent can be identified in the lower energy peak of the signal.

silicon substrate to the amorphous carbon sublayer is not clearly visible, but can be estimated from the difference of the increase of the carbon and gold signals in the EDX line scan (see Figure 7.10). However, the EDX line scan does not verify a variation of the concentration throughout the depth of the film.

The HRTEM micrograph shown in Figure 7.9 is taken from the area in the middle of the film (differently oriented - the border between the fifth and sixth layer runs vertically through the image). From this and further HRTEM images, the mean cluster diameter can be obtained. Within the different layers mean cluster sizes between $1.78 \pm 0.12 \mathrm{~nm}$ and $3.33 \pm 0.23 \mathrm{~nm}$ are found, with smaller cluster diameters in the brighter layers than in the darker layers and decreasing mean cluster size towards the surface. The RBS spectrum of sample s034 is given in Figure 7.11. In contrast to the EDX line scan, the gold signal in the RBS spectrum exhibits an explicit double peak structure. The smaller intensity of the gold signal at higher energies corresponds to the thicker bright layers in direction of the sample surface, while the stronger intensity at lower energies corresponds to the thicker dark layers towards the substrate. A small dent can be seen in the lower energy peak corresponding to the thin brighter layer between the two thicker dark layers.

Sample s035 was deposited with a slightly higher ion energy of $200 \mathrm{eV}$ for both carbon and gold ions. This sample shows no layer structure in TEM micrographs 

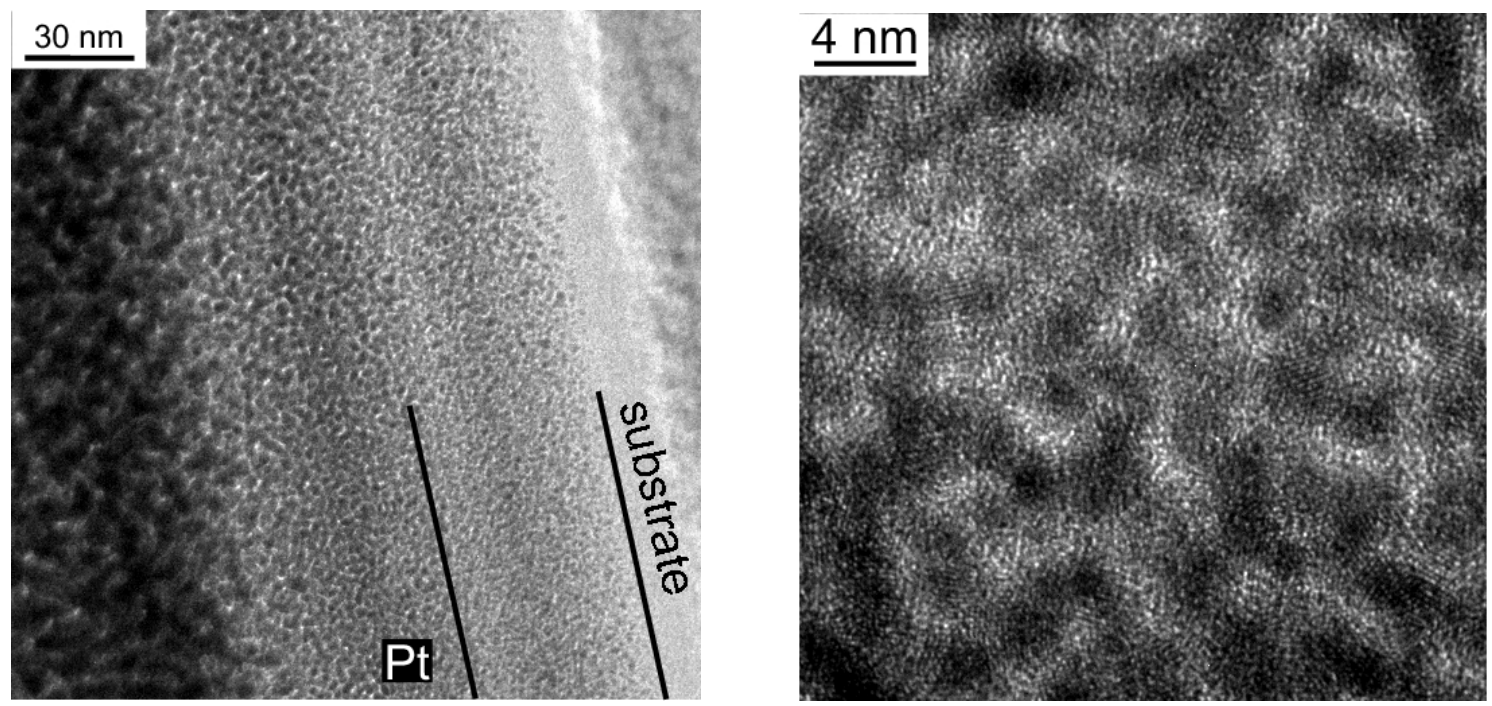

Figure 7.12: TEM micrograph of the a-C:Au sample s035 $\left(r_{\text {fluence }}=4, E_{\text {ion }}=200 \mathrm{eV}\right)$. The a-C:Au film appears to contain a uniform distribution of clusters embedded in an amorphous carbon matrix.

(see Figure 7.12) and no variation of the concentration can be seen in the EDX signal (not shown). It is evident that this sample is overproportionally thinner than sample s034. This can be attributed to enhanced sputtering at this higher ion energy. From HRTEM micrographs a mean cluster diameter of $2.64 \pm 0.13 \mathrm{~nm}$ can be derived.

The results of the composition analyses by RBS are displayed in Figure 7.13. There is no clear correlation between the film composition and the carbon/gold fluence ratio. For samples grown with the same deposition parameters, the gold concentration differs by up to a factor of two. However, a general trend comparable to the a-C:Cu films can be seen: with rising gold ion fluence fraction, the gold concentration of the films also increases. All samples have a rather severe shortage of gold as compared to the gold fluence fraction during deposition in common. Such losses suggest that gold atoms segregate at the film surface during deposition, and therefore the effective sputtering yield is increased.

A general gold cluster size dependency can be found in analogy to the a-C:Cu samples described in Section 5.2 (see Figure 7.14): At smaller gold contents, the cluster sizes are smaller. In particular, a corresponding variation of the cluster size between brighter and darker layers was found for sample s034. 


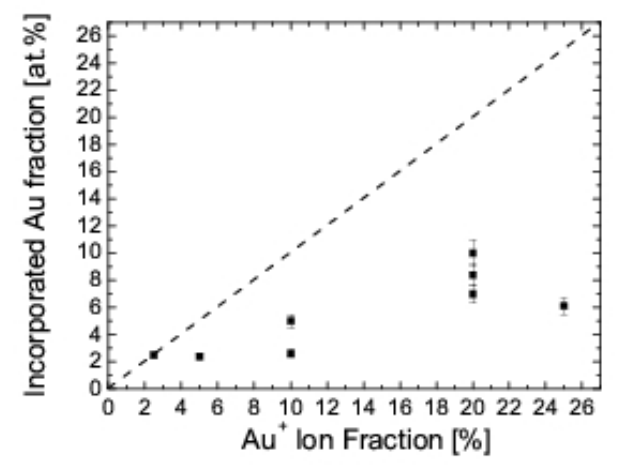

Figure 7.13: RBS results of the a-C:Au films. The gold concentration rises in tendency with the gold ion fluence fraction, but the deviations at a specific gold ion fluence fraction are rather large.

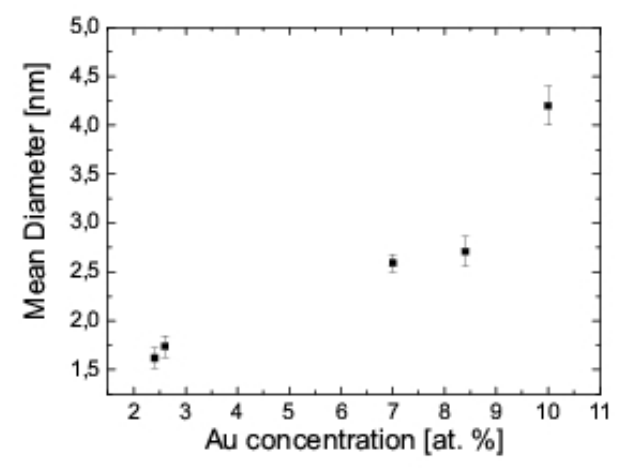

Figure 7.14: The mean gold cluster diameters rise with the gold concentration of the films.

\subsection{Amorphous Carbon Containing Iron}

Although the C-Fe system is very different from the C-Au system (see Section 2.2), these films show similar layer structures. The films were grown using the MSIBD setup Adonis. The sources were fed with $\mathrm{FeCl}_{3}$ and $\mathrm{CO}_{2}$ gas. The deposition parameters are listed in Table 7.4.

Figure 7.15 shows a TEM micrograph of sample 787 deposited at a carbon/iron fluence ratio of $r_{\text {fluence }}=4$. The dark layer near the substrate interface is a siliconcarbon-iron mixture phase similar to the silicon-carbon-metal mixture phases described above for the a-C:Au films. EDX confirms that the subsequent brighter layers are deficient in iron compared to the iron-rich darker layers. From TEM and

Table 7.4: Deposition parameters of the a-C:Fe samples. All samples were deposited at room temperature onto $\mathrm{p}-\mathrm{Si}<100>$ substrates.

\begin{tabular}{ccccc}
\hline \hline Sample No. & $r_{\text {fluence }}$ & $E_{\text {ion }}\left(\mathrm{C}^{+}\right)[\mathrm{eV}]$ & $E_{\text {ion }}\left(\mathrm{Fe}^{+}\right)[\mathrm{eV}]$ & Deposited charge $[\mathrm{C}]$ \\
\hline 800 & 99 & 100 & 40 & 0.061 \\
765 & 19 & 100 & 40 & 0.083 \\
797 & 9 & 100 & 40 & 0.102 \\
$772 / 787$ & 4 & 100 & 40 & $0.053 / 0.085$ \\
802 & 2.33 & 100 & 40 & 0.1 \\
\hline \hline
\end{tabular}



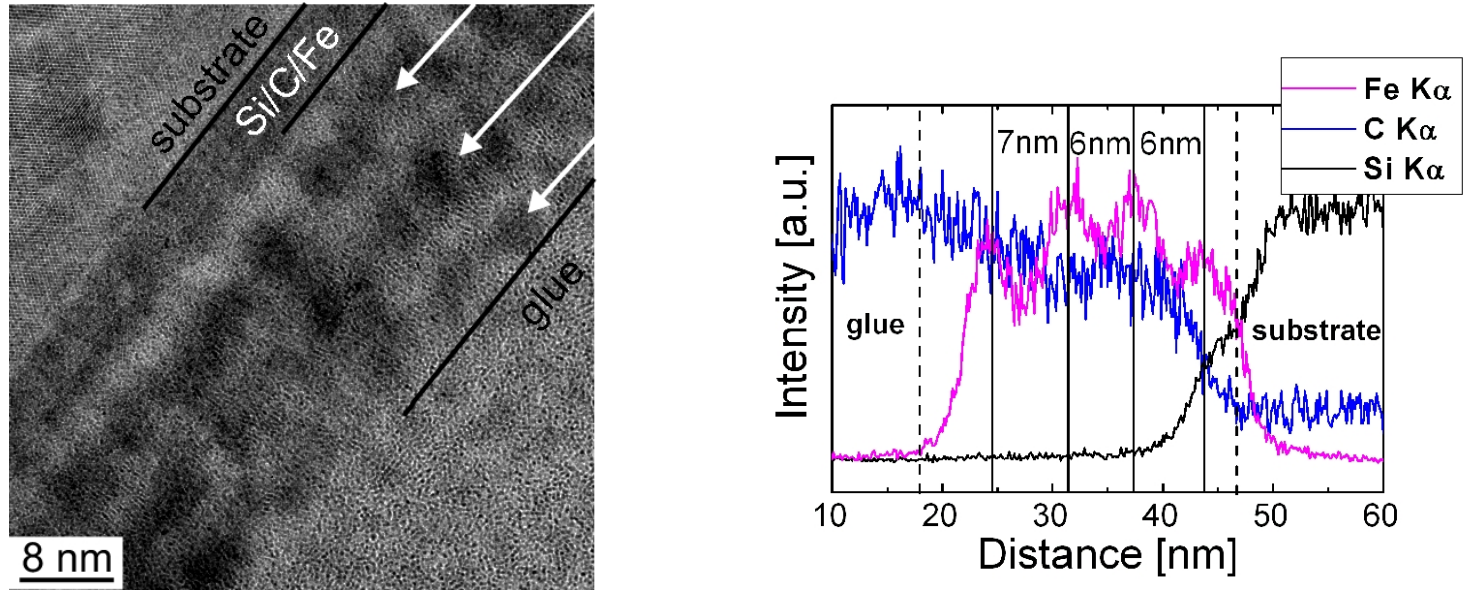

Figure 7.15: TEM micrograph of the a-C:Fe sample $787\left(r_{\text {fluence }}=4\right)$. The darker stripes contain a larger number of crystalline iron carbide clusters, whereas the brighter areas consist of amorphous carbon. The iron signal in the EDX line scan varies periodically over the whole depth of the film, clearly exhibiting four maxima with distances of 6-7 nm (from [152]). The maxima of the iron signal correspond to the darker layers in the micrograph.
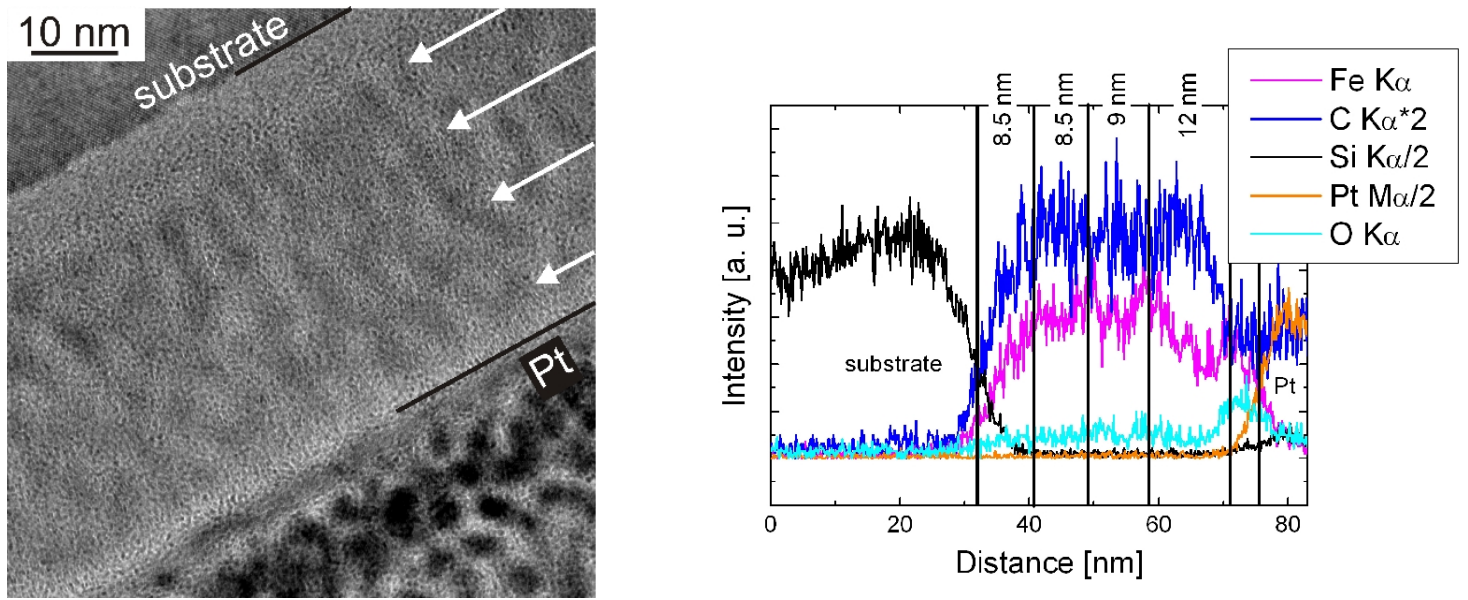

Figure 7.16: TEM micrograph of the a-C:Fe sample $797\left(r_{\text {fluence }}=9\right)$. A very slight indication of multilayers can be distinguished in the micrograph. The iron signal in the EDX line scan across the film also shows a small variation over the depth of the film, with maxima of 8-12 nm distance. The maxima of the iron signal are indicated by arrows in the micrograph. 


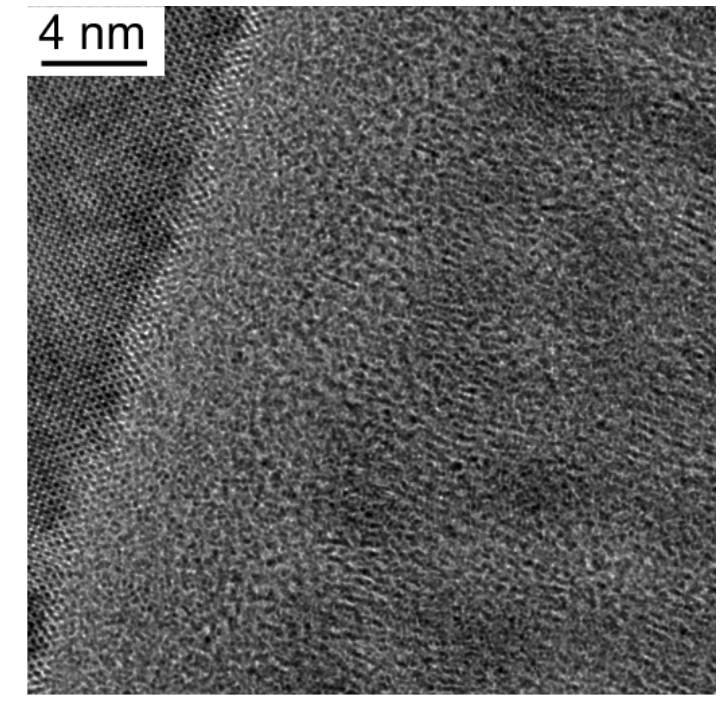

Figure 7.17: HRTEM image of the aC:Fe sample 797. The clusters appear amorphous and of a prolate shape oriented along the growth direction.

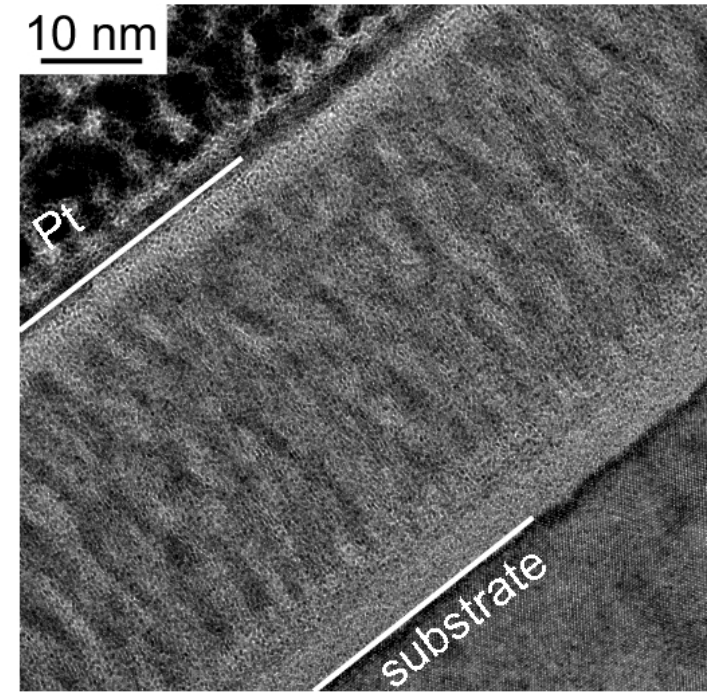

Figure 7.18: TEM image of the back side of the a-C:Fe sample 797. In this overview micrograph, an indication of a columnar structure can be seen.

the EDX line scan one can derive the period of the iron-rich layers to about 6-7 nm. The iron-rich dark layers are again composed of nanometre size crystals of prolate shape. Some micrographs give the impression of an alignment of the long axis along the growth direction. From others, the crystallites appear disordered. The mean value of the long axis of the particles of sample 787 is $a=4.51 \pm 0.12 \mathrm{~nm}$, the mean short axis is $b=3.06 \pm 0.07 \mathrm{~nm}$. Fourier transforms deliver a lattice plane spacing of $0.21 \mathrm{~nm}$. This strongly disagrees with the lattice plane spacings of $\alpha$-iron.

Sample 797 was prepared for TEM using the focused ion beam setup. The overview micrograph and EDX line scan are shown in Figure 7.16. Again, the film surface is indicated by the interface to the platinum cover layer. This image obtains only a slight indication of a multilayer structure. The EDX line scan confirms a weak variation of the iron content. The maxima of the iron signal are indicated by arrows in the micrograph. These maxima are a little further apart from each other than the ones of sample 787. The distances are between 8.5 and $12 \mathrm{~nm}$. The mixture layer of iron, carbon and silicon is not discernible. Figure 7.17 shows a HRTEM micrograph of sample 797. All the clusters appear amorphous and are of prolate shape with the long axis aligned along the growth direction. The mean long and short cluster diameters are $a=3.97 \pm 0.14 \mathrm{~nm}$ and $b=2.86 \pm 0.11 \mathrm{~nm}$, respectively. The prolate shape of the clusters leads to another striking feature of sample 797 . It 


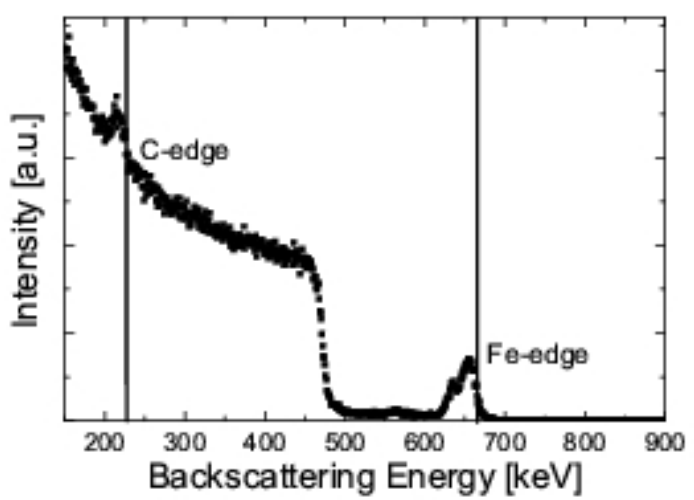

Figure 7.19: RBS spectrum of the a-C:Fe sample $765\left(r_{\text {fluence }}=19\right)$. The iron signal shows a weak double peak structure.

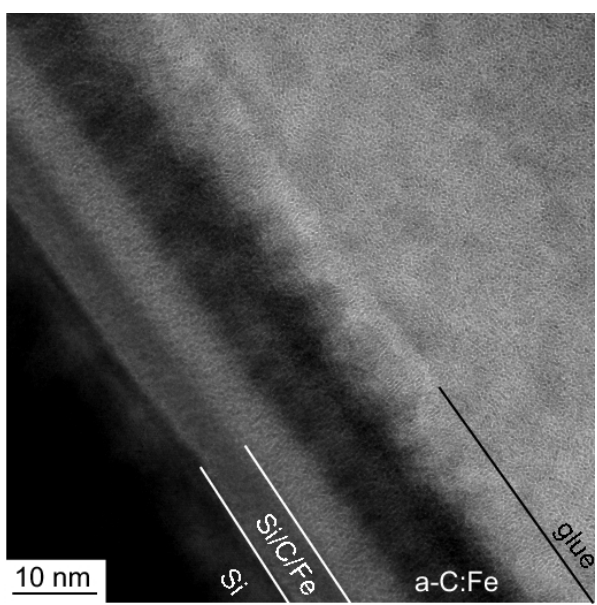

Figure 7.20: TEM micrograph of the a-C:Fe sample 765. The film consists of an iron-rich layer embedded in iron-deficient amorphous carbon layers.

shows an indication of a columnar structure due to an alignment of several prolate clusters in direction perpendicular to the surface. This becomes especially evident in the overview micrograph displayed in Figure 7.18 , This image was recorded from the backside of the sample (the sample was turned over and re-introduced into the microscope).

Not all of the RBS spectra measured for the a-C:Fe films show a double peak structure of the metal signal as it is the case for most of the a-C:Au films. A double peak iron signal is only observed in the RBS spectrum of sample 765 (see Figure

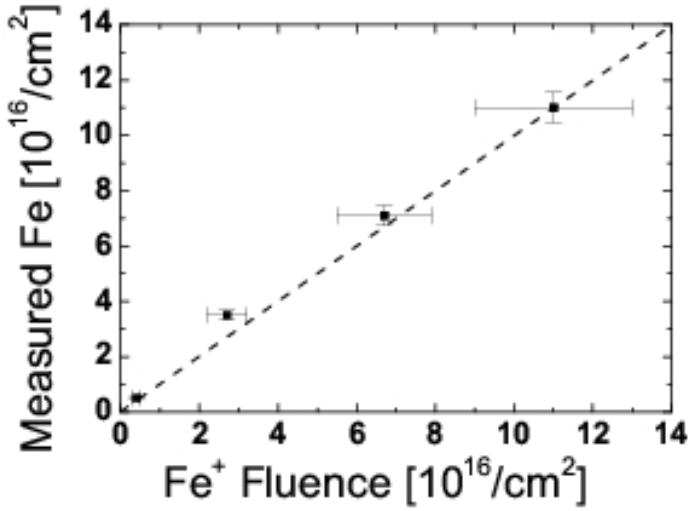

Figure 7.21: RBS results of the aC:Fe films. The iron areal atomic density of the films is given in dependence on the deposited iron ion fluence. All deposited iron atoms are incorporated into the films. Apparently, iron is not subject to sputtering and the sputtering yield is $S_{F e}=0$ for all samples (from [152]). 
7.19). For higher iron contents, the distances between the layers are too narrow to be resolved by RBS. In Figure 7.19, the peak at smaller backscattering energies resembles the silicon-carbon-iron mixture layer. The peak at higher energies arises from an iron-rich layer separated from the substrate by an iron-deficient layer. This structure is in accordance with the TEM micrograph of the sample (see Figure 7.20). The TEM image shows that there are two bright layers both above and underneath the dark iron-rich film.

The iron areal densities of the films measured by RBS resemble the deposited iron ion fluences (see Figure 7.21). The sputtering yields of iron obtained from SRIM simulations [112] are in the range of 0.26 and 0.33 , considering a surface composition according to the iron ion fraction and taking the carbon/iron fluence ratios into account. As the experiment results in a sputtering yield of $S_{F e}=0$ for all a-C:Fe samples and no sputtering of iron atoms off the film surfaces occurs, it is deduced that the surface consists of only carbon atoms during the entire deposition process.

\subsection{Summary and Discussion}

The influence of the copper ion energy on the film structure was investigated. As samples deposited with the lower carbon ion energy of $80 \mathrm{eV}$ obtained relatively small fractions of $\mathrm{sp}^{3}$ bonds, the ion energy was raised to $100 \mathrm{eV}$, although this also yields a higher sputtering yield for copper. At higher copper ion energies, only small fractions of the deposited copper is incorporated in the film. No increased sputtering is found for the comparison sample that was grown at a copper ion energy of $40 \mathrm{eV}$ and an ion fluence ratio of 4 . At these parameters, the sputtering yields are rather low. However, at higher copper energies, the copper atoms are subjected to more severe sputtering. The sputtering losses are slightly or sometimes even much stronger than expected from the carbon/copper fluence ratio and the proposed surface composition according to the copper ion fraction. It is therefore suggested that a certain fraction larger than the copper ion fraction must therefore segregate at the film surface during deposition. Due to the lack of copper, no clusters were found in TEM micrographs at higher copper ion energies. The matrix contained a slightly higher fraction of $\mathrm{sp}^{3}$ bonds than for the films grown with a carbon ion energy of $80 \mathrm{eV}$ and show otherwise the same dependence on the carbon/copper fluence ratio. There are much smaller amounts of metal actually present in the samples, but the $\mathrm{sp}^{3}$-bonding content is 
nevertheless decreased. This is rather astonishing, as it is sometimes argued that the increased $\mathrm{sp}^{2}$-bonding fraction is concentrated at the cluster/matrix interfaces. This is obviously not the case.

A variation of the film structure in growth direction was found for a-C:Au films. However, the multilayer structure of sample s007 cannot be reproduced in detail, and rather less pronounced layers result for samples grown under the same deposition conditions. In the case of sample s034, which was prepared for TEM analysis using a focused ion beam setup, the multilayer structure may have been blurred in the preparation process. The use of the focused ion beam results in a more severe damage of the material than is imposed by the ion mill used in conventional TEM sample preparation. This hypothesis is supported by the clear double peak structure seen in RBS.

The increase of the carbon and gold ion energies to $200 \mathrm{eV}$ resulted in a homogeneous distribution of gold clusters throughout an amorphous carbon matrix. When the energy is raised, two processes lead to the suppression of a multilayer morphology: First, the gold ions are implanted deeper into the film and only smaller fractions reach the surface. Second, the sputtering yield becomes larger and therefore any gold atoms segregating at the surface are eventually sputtered off. The evolution of a multilayer structure may therefore be established only in a small and rather well defined ion energy range. This could also explain the less pronounced layers for sample s009 if the conditions in the ions source during deposition yielded a broader ion energy distribution.

The mean cluster diameters are in the range of 1.6 to $5 \mathrm{~nm}$. The sizes rise with the gold concentration in the film. This is a reasonable behaviour, as there is a larger number of gold atoms within a volume according to an ion-induced diffusion length around the cluster.

A pronounced multilayer structure with rather periodic variations was also found in the a-C:Fe sample 787. The iron concentration varies over the depth of the sample and iron-rich layers consist of crystalline particles surrounded by amorphous carbon. For smaller iron contents, as for samples 797 and 765, an indication of the formation of multilayers can be seen. In particular, for sample 797, which was deposited at a carbon/iron fluence ratio of $r_{\text {fluence }}=9$, the period of the layers is increased as compared to sample 787 , which was deposited with $r_{\text {fluence }}=4$. The rather rudimentary variation may be caused by the preparation using the focused ion beam system. 
The prolate shape of the iron clusters, as it was found in sample 787, may be generated by the internal compressive stress that is typical for ion beam deposited films. Under the influence of the gallium irradiation in the focused ion beam setup, this effect may be enhanced and therefore result in the columnar structure of sample 797. A surface diffusion and island growth generation of such a prolate shape, as is was proposed by Babonneau and co-workers [42], can be ruled out: From the RBS and TEM analyses it is evident, that the iron stays within the film and does not segregate at the surface.

The unequal results for samples of similar deposition conditions but prepared by different techniques (conventional mechanical TEM preparation or focused ion beam) demand more thorough comparative studies. It is essential to ensure that the focused ion beam has no influence on the film morphology. 


\section{Chapter 8}

\section{Model for a-C:Me Structure Formation During the Deposition of Hyperthermal Species}

In order to understand the structure formation during ion beam deposition, an atomic transport mechanism inside the film, which can either be thermally activated or ion-induced, has to be taken into account. All the samples were grown at room temperature. The ion current densities were low $\left(<15 \mu \mathrm{A} / \mathrm{cm}^{2}\right)$ and did not cause any global heating of the sample. As summarized in Section 5.1, previous studies by Kröger et al. [128] showed no diffusion of copper and silver and several other elements upon thermal treatment up to $1000^{\circ} \mathrm{C}$ within amorphous carbon environments. Thus, thermally activated diffusion of atoms within the films and Ostwald ripening, a basic process leading to cluster formation, can be excluded. Instead, an ion-induced transport process is assumed as was introduced in Section 3.4. Due to atomic collisions and the excitation of phonons resulting in a thermal spike, an incoming ion severely changes the atomic structure of the close environment of its path. For the ion energies chosen in the experiment, the cylindrical thermal spike model suggests significant atomic rearrangements along the length of the ion path $r_{I}(\sim 1-3 \mathrm{~nm})$ during a time scale of about $10^{-12} \mathrm{~s}$, before the impact energy is dissipated. The value of the fraction of rearrangement processes per atom in the spike volume, as it can be gained from Equation (3.6), is approximately $n_{T} / n_{S}=1$. For the diffusion of the metal atoms, this can be treated as a random walk process, where each diffusion step is triggered by a single ion impact. A given metal atom in the collision cascade volume is rearranged approximately once for each ion impact process, and it is assumed that it travels a distance of one typical atom spacing $a$ 
$(\approx 0.15 \mathrm{~nm}$ for amorphous carbon). The cylindrical thermal spike model predicts typically 10-20 rearranged atoms per ion impact for the ion energies regarded here. Once the film thickness has increased by the mean ion range $r_{I}$, the successive ion impacts have resulted in about $N \sim 10^{2}-10^{3}$ rearrangement steps of a particular atom. If a uniform three-dimensional random walk diffusion is assumed [153], the average travelled distance $\bar{x}=\sqrt{3 N a^{2}}$ results in 2-5 nm. This ion-induced diffusion length is sufficient to account for the cluster formation within an amorphous carbon matrix and it is a determining factor of the final cluster diameters. However, if the small metal clusters that form in the course of the film growth are not stable against further ion impact, the metal atoms are dispersed and the diffusion direction is preferentially oriented towards the surface. In this case, the mean travelled distance is increased, because the regarded atom stays in the area effected by ion impacts. Therefore, an ongoing transport of atoms to the film surface is also possible solely generated by ion-induced diffusion.

The processes involved in the deposition process are far from thermodynamic equilibrium and the material does not resemble a supercooled liquid at any stage. A description on the basis of spinodal decomposition in the bulk of the film is therefore not applicable. Furthermore, spinodal decomposition would not be able to account for the formation of a multilayer structure in the a-C:Au and a-C:Fe case on the one hand and a homogeneous cluster formation, as it was found for a-C:Cu films [14] on the other hand.

The following sections will describe, how the atomic transport processes described above may lead to the various structures taking three major determining factors into account: first, the miscibility of the phases involved; second, the stability of the small clusters formed during the deposition process; and third, the sputtering yield of the respective elements under certain conditions.

\subsection{The Uniform Cluster Distribution of a-C:Cu films}

As described in Section 5.2, a-C:Cu films deposited using MSIBD consist of nanometre-sized copper clusters distributed uniformly within an amorphous carbon matrix. The mean cluster diameter is tunable between about $3.5 \mathrm{~nm}$ and $9 \mathrm{~nm}$ by selecting different ion fluence ratios $r_{\text {fluence }}=19 \ldots 1$.

Since copper is immiscible with carbon, the formation of precipitates is energeti- 
cally favourable. According to the transport processes discussed above, it is assumed that a copper atom within the film moves one typical atom spacing into a random direction with each ion impact in its immediate neighbourhood. When it meets another copper atom, a dimer will be formed. This dimer (or a cluster of a small number of atoms that is formed in a subsequent process) is assumed to be stable under further ion impacts. Otherwise, a segregation of copper at the film surface would be expected. This scenario is also supported by the experiment by Chey et al. described in 2.3 , where small copper clusters were attempted to be moved across a $\operatorname{Si}(111)-(7 \times 7)$ surface using the tip of a scanning tunnelling microscope (STM) [95]. The copper clusters could not be moved and broke, when the applied force was too strong. Furthermore, the theoretical analyses of copper clusters summarized in Section 2.3 suggest that they are rather rigid. The clusters can grow as newly deposited copper atoms encounter them in their ion-triggered random walk. A rather homogeneous cluster distribution within the amorphous carbon matrix arises. The mean cluster size is related to the average metal concentration provided by the carbon/copper fluence ratio and the ion-induced diffusion length $\bar{x}$ of copper atoms. In the regarded concentration range, the cluster diameters are expected to be of the order of a few nanometres, as $\bar{x} \approx 2-5 \mathrm{~nm}$. This is confirmed by TEM of the a-C:Cu films.

The experimental analysis of the sputtering yield for the a-C:Cu films grown with a higher carbon ion energy and at various copper ion energies reported on in Section 7.1 result in a slightly overproportionate loss of copper from the films. This suggests that the small copper clusters formed during the deposition process are partially destroyed when the copper ion energy is raised and copper segregates at the surface yielding a copper surface concentration larger than the copper ion fraction. In such cases, the morphology of the film may evolve in a manner as it is presented in the following for a-C:Ag and a-C:Au films.

\subsection{Surface Segregation and Multilayer Formation}

The co-deposition of $\mathrm{C}^{+}$and $\mathrm{Ag}^{+}$ions using MSIBD resulted in a complete segregation of the silver atoms at the surface, where they either formed clusters or were nearly completely sputtered off the film, as was described in Section 5.3. It is therefore assumed that small silver clusters that may have formed in the ion-triggered 


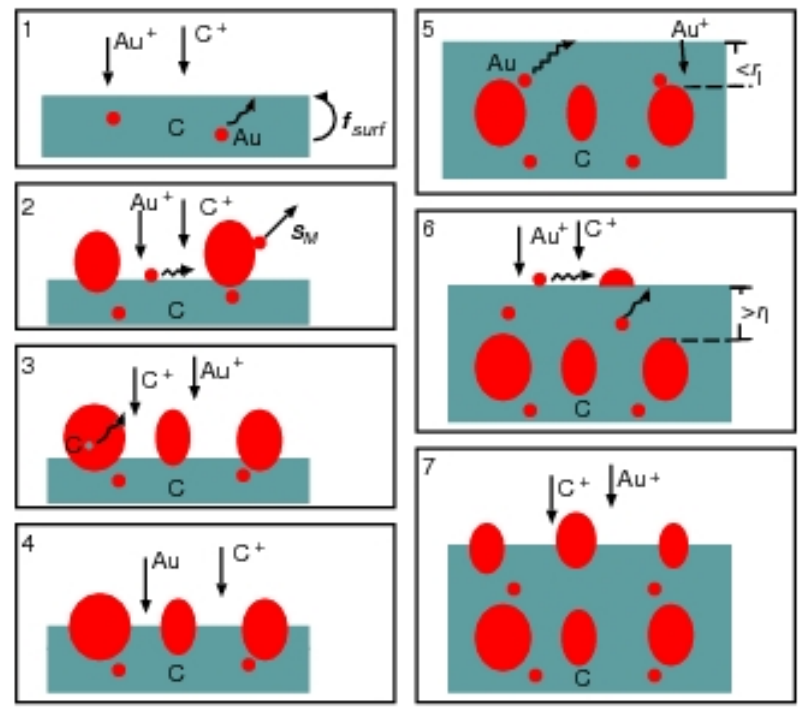

Figure 8.1: Schematic of the multilayer formation process.

diffusion process do not resist successive ion impacts. The silver atoms are dispersed and eventually transported towards the surface. This assumption is in agreement with the above mentioned experiment by Chey et al., where the attempt to move silver clusters across the $\operatorname{Si}(111)-(7 \times 7)$ surfaces with the STM tip was successful and resulted in a track of silver atoms along the path the cluster was moved 95. Furthermore, the theoretical analysis shows that silver clusters can be regarded as liquid droplets in contrast to the rigid copper clusters (see Section 2.3).

The scenario leading to the formation of alternating metal-deficient and metalrich layers is schematically depicted in Figure 8.1. Due to successive ion impacts, the silver atoms are eventually transported towards the surface. The driving forces behind the surface segregation are most likely the density gradient and the compressive stress that is characteristic of ion beam deposited films. This scenario should also apply to the case of the a-C:Au films, as small gold clusters can also be described by a liquid drop model [154].

The fraction $f_{\text {surf }}$ of silver or gold atoms that reach the sample surface in the course of ion impact induced diffusion are then subjected to severe sputtering. Considering a given metal that segregates at the surface, there are two possible cases: either the sputtering yield $S_{M}$ is greater than 1 . Then, all metal atoms are sputtered off the film. Or the sputtering yield depending on the ion fluence ratio and the ion energies is $S_{M}<1$. This results in an accumulation of metal atoms at the surface, 
as the supply of metal atoms by the incident ion beam exceeds the sputtering loss. As described in Section 2.4 the supply of metals to the surface results in a growth of metal islands on the surface due to surface diffusion. The diffusion on the surface may even be enhanced by the irradiation with ions (see Section 3.4). In this way, the metal clusters on the surface grow to a size where they cannot be considered as liquid droplets anymore and become rigid.

While the clusters grow on the film surface, incoming $\mathrm{C}^{+}$ions contribute to the growth of a carbon layer underneath. Any carbon that is implanted into a cluster is transported out towards the amorphous carbon film, because carbon is not soluble in silver or gold. Eventually, spaces in between clusters are filled with carbon and the cluster layer is buried underneath a newly forming carbon layer. As long as the incoming metal ions reach the buried clusters when being subplanted into the film or during their rearrangement steps, they further contribute to the cluster growth of the buried layer. If not, they will again segregate at the newly forming carbon film surface. There, they form a new layer of metal clusters while incoming $\mathrm{C}^{+}$ions contribute to the growth of a metal deficient layer separating the cluster layers. The growth of the clusters of one layer is complete when the thickness of the film above exceeds the ion range $r_{I}$.

This scenario may apply in general to both the a-C:Au and the a-C:Ag case. If the sputtering yield $S_{M}$ depending on the material, the ion fluence ratio, and the ion energies approaches a value of $S_{M} \approx 1$, the metal-rich layers are separated by thicker metal-deficient layers compared to the ones where $S_{M}$ is smaller. In the case of the a-C:Ag films described in Section 5.3 where the sputtering yield reaches values of up to $S_{M}=0.95$, the layer period is larger than the total film thickness, resulting in just a single layer of silver clusters on top of a silver-depleted amorphous carbon film.

\subsection{Multilayer formation of a-C:Fe films}

The mechanisms involved in the formation of multilayers in a-C:Fe films are in part the same as described above for the a-C:Au films. Iron carbides are rich in iron (we assume that the most frequent $\mathrm{Fe}_{3} \mathrm{C}$ phase is formed) [86], whereas the samples reported on in this thesis bear an average iron content of only 5-20 at.\%. Therefore, iron carbide clusters embedded in an amorphous carbon matrix will form. The deposited iron accumulates in a buried iron carbide layer by ion-induced transport 
until the $\mathrm{Fe}_{3} \mathrm{C}$ stoichiometry is reached and stable $\mathrm{Fe}_{3} \mathrm{C}$ clusters are formed. The excess carbon atoms are transported out of the $\mathrm{Fe}_{3} \mathrm{C}$ layers due to ion-induced rearrangements, most likely towards the surface. As a result, a pure carbon surface layer emerges. No iron atoms are lost due to sputtering, because they are not part of the surface during the entire deposition process. This is in agreement with the measured sputtering yield of $S_{F e}=0$. With increasing thickness of the amorphous carbon surface layer, the incoming iron ions cannot reach the buried iron carbide cluster layer anymore, and a new iron carbide layer grows, separated from the previous one by a thin carbon-rich layer. As a result, a multilayer film structure evolves.

\subsection{Sputtering Losses}

In the energy regime of the ions used for this work, the sputtering of carbon atoms is negligible. We therefore have to concentrate on the loss of metal atoms due to sputtering. Under steady-state conditions, the sputtering yield for metal atoms per incoming metal ion is given by

$$
S_{M}=r_{\text {fluence }} s_{C M}+s_{M M}
$$

where $s_{C M}$ and $s_{M M}$ are the sputtering yields of metal atoms due to impinging carbon ions and the respective metal ions if a pure metal surface is assumed. The values for $s_{C M}$ and $s_{M M}$ were obtained from SRIM calculations [112] and are listed in Table 8.1. The number of metal atoms sputtered per incoming metal ion can then be calculated using Equation (8.1). The results are presented in Table 8.2.

The sputtering loss of metal atoms, however, also depends on the respective average surface concentration $c_{\text {surf }}$, which should be proportional to the metal ion fraction $f_{M}$ in the case of isotropic ion-triggered diffusion. From the RBS analysis of the a-C:Cu samples [14], we find an average sputtering loss of $\overline{S_{M}}=0.21 \pm 0.05$. A

Table 8.1: SRIM calculated self-sputtering yields $s_{M M}(\mathrm{M}=\mathrm{Cu}, \mathrm{Ag}, \mathrm{Au}, \mathrm{Fe})$ and sputtering yields $s_{C M}$ of metal atoms by incident carbon ions of the respective energies.

\begin{tabular}{cccccccc}
\hline \hline $\begin{array}{c}\text { Impinging } \\
\text { Ion }\end{array}$ & $s_{C u C u}$ & Impinging & $s_{A g A g}$ & Impinging & $s_{A u A u}$ & Impinging & $s_{F e F e}$ \\
Ion & $s_{C C u}$ & Ion & $s_{C A g}$ & Ion & $s_{C A u}$ & Ion & $s_{C F e}$ \\
\hline $40 \mathrm{eV} \mathrm{Cu}^{+}$ & 0.10 & $60 \mathrm{eV} \mathrm{Ag}^{+}$ & 0.18 & $100 \mathrm{eV} \mathrm{Au}^{+}$ & 0.37 & $40 \mathrm{eV} \mathrm{Fe}^{+}$ & 0.05 \\
$80 \mathrm{eV} \mathrm{C}^{+}$ & 0.22 & $80 \mathrm{eV} \mathrm{C}^{+}$ & 0.04 & $100 \mathrm{eV} \mathrm{C}^{+}$ & 0.03 & $100 \mathrm{eV} \mathrm{C}^{+}$ & 0.32 \\
\hline \hline
\end{tabular}


Table 8.2: Total sputtering yield induced by both carbon and metal ions. The values $S_{M}^{S R I M}=r_{\text {fluence }} s_{C M}+s_{M M}$ were calculated using the sputtering yields given in Table 8.1. The $S_{M}^{R B S}$ values were determined from RBS measurements. In the a-C:Cu case, these have to be compared with the values of $c_{\text {surf }} S_{M}^{S R I M}$, as the sample surface contains only a fraction $c_{\text {surf }}=f_{M}=f_{C} / r_{\text {fluence }}$ of copper atoms.

\begin{tabular}{cccccccc}
\hline \hline & & \multicolumn{2}{c}{$\mathrm{Cu}$} & & \multicolumn{2}{c}{$\mathrm{Ag}$} & \multicolumn{2}{c}{$\mathrm{Au}$} \\
$r_{\text {fluence }}$ & $S_{M}^{S R I M}$ & $S_{M}^{R B S}$ & $c_{\text {surf }} S_{M}^{S R I M}$ & $S_{M}^{S R I M}$ & $S_{M}^{R B S}$ & $S_{M}^{S R I M}$ & $S_{M}^{R B S}$ \\
\hline 19 & & & & 0.94 & $0.90 \pm 0.04$ & 0.94 & $0.50 \pm 0.03$ \\
9 & 2.08 & $0.38 \pm 0.10$ & 0.21 & 0.54 & $0.95 \pm 0.04$ & 0.64 & $0.74 \pm 0.04$ \\
3 & & & & & & 0.46 & $0.76 \pm 0.04$ \\
4 & 0.98 & $0.26 \pm 0.11$ & 0.20 & 0.34 & $0.69 \pm 0.04$ & 0.49 & $0.50 \pm 0.03$ \\
2.33 & 0.61 & $0.12 \pm 0.14$ & 0.18 & 0.27 & $0.44 \pm 0.04$ & & \\
1.5 & 0.43 & $0.20 \pm 0.13$ & 0.17 & & & & \\
1 & 0.32 & $0.10 \pm 0.14$ & 0.16 & & & & \\
\hline \hline
\end{tabular}

dependence on the metal ion fraction $f_{M}$ cannot be claimed from the RBS results, as the errors are too large. However, the experimental values are in accordance with the calculated values $c_{\text {surf }} S_{M}^{S R I M}$, as shown in Table 8.2 .

For both a-C:Ag and a-C:Au films, RBS analysis reveals larger sputtering losses of metal atoms than for the a-C:Cu films. The measured sputtering losses vary between $0.44 \pm 0.04$ and $0.9 \pm 0.04$ for silver and about $0.5 \pm 0.03$ and $0.76 \pm 0.03$ for gold. This confirms that both metals segregate at the film surfaces and therefore a strongly increased amount of metal atoms is subjected to sputtering. Assuming that all metal atoms reach the surface, the sputtering yields gained from RBS have to be compared directly to the values calculated from Equation (8.1) using SRIM simulations.

\subsection{Estimation of the Multilayer Period for Metal Surface Segregation}

Now, the period of the layer structures for the case of metal surface segregation, i.e. the a-C:Ag and a-C:Au films, will be estimated based on sputter yield data and ion fluences. The films were deposited at a carbon/metal fluence ratio $r_{\text {fluence }}$. We apply the metal's bulk atomic density $n_{M}$ and the bulk density of graphite $n_{C}$. The total sputtering yield $S_{M}$ of the metal per incoming metal ion is extracted from RBS measurements and can for comparison be calculated from the SRIM data by 
Equation (8.1). The sputtering yield of carbon by metal ions is zero for all conditions under investigation and the sputtering yield of carbon by carbon ions $s_{C C} \approx 0.1$. The covering of the carbon layer with a layer of metal clusters may be incomplete, which is described by the covering factor $c$. The thickness $d_{M}$ of the layer of metal clusters is taken from experimental data (TEM micrographs). The areal density $N_{M}$ in this metal cluster layer is $N_{M}=n_{M} d_{M} c$. Using this, the necessary total metal fluence $F_{M}$ that has to be deposited in order to grow one metal cluster layer and account for sputtering losses and the fraction of dispersed metal atoms that remain within the matrix is calculated, where $f_{\text {surf }}$ gives the fraction of deposited metal atoms segregating at the surface:

$$
F_{M}=\frac{N_{M}}{\left(1-S_{M}\right) f_{\text {surf }}}=\frac{n_{M} d_{M} c}{\left(1-S_{M}\right) f_{\text {surf }}} .
$$

The corresponding total fluence of carbon atoms is larger than $F_{M}$ by a factor of the carbon/metal fluence ratio $r_{\text {fluence }}$ :

$$
F_{C}=r_{\text {fluence }} F_{M}
$$

The deposition of this amount in addition to the fraction $\left(1-f_{\text {surf }}\right)$ of dispersed metal atoms and lacking the amount of sputtered carbon atoms leads to a thickness $d_{C}$ of the carbon layer of

$$
d_{C}=\frac{F_{C}\left(1-s_{C C}\right)}{n_{C}}+\frac{F_{M}\left(1-f_{\text {surf }}\right)}{n_{m}} .
$$

Applying Equations (8.2) and (8.3) to Equation (8.4), the period $t=d_{M}+d_{C}$ results in

$$
t=d_{M}\left[1+\frac{n_{M} c}{\left(1-S_{M}\right) f_{\text {surf }}} \times\left(\frac{1}{n_{C}} r_{\text {fluence }}\left(1-s_{C C}\right)+\frac{1}{n_{M}}\left(1-f_{\text {surf } f}\right)\right)\right] .
$$

This expression can be simplified if a full segregation of the metal atoms at the surface, a complete coverage for the metal cluster layer, and negligible carbon sputtering is assumed $\left(f_{\text {surf }}=1, c=1, s_{C C}=0\right)$ :

$$
t \approx d_{M}\left[1+\frac{n_{M}}{\left(1-S_{M}\right)} r_{\text {fluence }} \frac{1}{n_{C}}\right] .
$$

Instead of a rectangular metal cluster layer with thickness $d_{M}$, a layer with Gaussian concentration profile of the same areal density should be assumed. The FWHM value 
is taken from the TEM and EDX analysis. Therefore, $d_{M}=1.06 \cdot \mathrm{FWHM}$ has to be replaced in 8.5) or 8.6.

For the a-C:Au sample number s007, where $r_{\text {fluence }}=4, n_{M}=59 / \mathrm{nm}^{3}$, and $n_{C}=110 / \mathrm{nm}^{3}$, and $S_{M}=0.5$ and FWHM $=5-9 \mathrm{~nm}$ are derived from RBS and EDX analyses, a period of the layer structure of $t \approx 28 \mathrm{~nm}$ is obtained from Equation (8.6). From the TEM micrographs and EDX analysis one finds the experimental value $t_{\text {exp }} \approx 14 \mathrm{~nm}$. It is, however, most likely that the covering factor $c<1$. Although the TEM micrographs suggest complete layers of metal clusters, presumably clusters that are not in the interstice of two clusters but are rather behind this blank are seen. A comparison of the gold areal density determined by RBS $\left(\approx 4 \times 10^{16}\right)$ with the necessary amount to create a multilayer structure with cluster layers of gold bulk density $\left(1.6 \times 10^{17}\right)$ results in a value of $c \approx 0.25-0.3$ and a layer period $t$, which is in good agreement with the experiment.

\subsection{Estimation of the Multilayer Period for Carbide Forming Metals}

The period of the a-C:Fe multilayer structures can be estimated in analogy to the a-C:Au case, taking a few alterations into account. As the iron ions do not segregate at the film surface but accumulate in layers within the film, a parameter $f_{\text {layer }}$, which reflects the fraction of iron atoms accumulating in an iron carbide cluster layer, has to be introduced. The fraction $\left(1-f_{\text {layer }}\right)$ of iron atoms is dispersed throughout the carbon-rich layers. Furthermore, it has to be considered that the clusters of the metal-rich layers contain a significant amount of carbon which is not available for the separating carbon layers. If $n_{\mathrm{Fe}_{3} \mathrm{C}}=103 / \mathrm{nm}^{3}$ is the atomic density of $\mathrm{Fe}_{3} \mathrm{C}$, the iron fluence for one cluster layer covered with a fraction $c$ with clusters is

$$
F_{F e}=\frac{0.75 \cdot n_{\mathrm{Fe}_{3} \mathrm{C}} \cdot d_{M} \cdot c}{f_{\text {layer }}}
$$

which again corresponds to a deposited carbon ion fluence of

$$
F_{C}=r_{\text {fluence }} F_{F e} .
$$

The thickness $d_{C}$ of the carbon layer is reduced by the amount of carbon necessary to meet the $\mathrm{Fe}_{3} \mathrm{C}$ stoichiometry and the sputtering of carbon atoms. It is, however, increased by the dispersed iron atoms that do not add to the iron carbide cluster 
layers:

$$
d_{C}=F_{F e}\left(\frac{\left(r_{\text {fluence }}\left(1-s_{C C}\right)-0.25\right.}{n_{c}}+\frac{1-f_{\text {layer }}}{n_{F e}}\right),
$$

where $n_{C}=110 / \mathrm{nm}^{3}$ and $n_{F e}=85 / \mathrm{nm}^{3}$ are the atomic densities of graphite and iron, respectively. Applying Equation 8.7 to Equation (8.4) yields a period $t=$ $d_{M}+d_{C}$ of

$$
\begin{aligned}
t=d_{M} & {\left[1+\frac{0.75 \cdot n_{\mathrm{Fe}_{3} \mathrm{C}} \cdot c}{f_{\text {layer }}}\right.} \\
& \left.\times\left(\frac{r_{\text {fluence }}\left(1-s_{C C}\right)-0.25}{n_{c}}+\frac{1-f_{\text {layer }}}{n_{F e}}\right)\right] .
\end{aligned}
$$

Assuming a covering with metal clusters in each cluster layer of $c=0.7$ and that the fraction of iron atoms contributing to the cluster layers is $f_{\text {layer }}=0.7$, one gets $t=7 \mathrm{~nm}$ for the sample number 787 , where $d_{M} \approx 2 \mathrm{~nm}, r_{\text {fluence }}=4$. This is again in good agreement with the results derived from TEM and EDX.

\subsection{Predictions}

From Equation (8.5) it follows that the period of the layer structure strongly depends on the total sputtering yield of surface metal atoms $S_{M}=s_{M M}+r_{\text {fluence }} s_{C M} . S_{M}$ itself depends on the ion energies and the carbon/metal fluence ratio $r_{\text {fluence. }}$ A careful selection of these parameters would allow the growth of multilayer structures with variable layer periods. If, however, $S_{M}>1$, all metal atoms are sputtered off the film and no layer structure can arise. The film contains only small amounts of dispersed metal atoms or small metal clusters, depending on $f_{\text {surf }}$. In order to grow films with a uniform distribution of metal clusters within a diamond-like amorphous carbon matrix, the carbon ion energy has to be kept at about $100 \mathrm{eV}$ in order to achieve a high $\mathrm{sp}^{3}$-bonding content. The energy of the metal ions should be raised so that the mean ion range well exceeds the carbon ion range of about $1 \mathrm{~nm}$, and in addition $S_{M}>1$. The first condition increases the probability for metal atoms to remain within the amorphous carbon matrix and form clusters; the second one ensures that all surface metal atoms are completely sputtered off. The maximum metal ion energy is, however, limited to several hundred eV, because otherwise the sputtering yield of carbon by metal ions becomes significant. This scenario can be compared to the ion beam deposition of $1 \mathrm{keV}$ silver ions into tetrahedral amorphous carbon by Kröger and co-workers [128. They observed that about $10 \%$ 
of the deposited silver was incorporated in the film, corresponding to a fraction of $f_{\text {surf }}=0.9$ of the silver atoms segregating at the surface. As the sputtering yield

$S_{A g} \gg 1$ at the given circumstances, all silver reaching the surface is removed. As the deposition procedure is not a co-deposition process but rather implantation of only $\mathrm{Ag}^{+}$ions into a carbon film followed by the deposition of a carbon covering layer, the result is a buried layer of silver.

For the case of carbon surface segregation, sputtering plays only a minor role. Here, the superfluous carbon atoms are transported towards the surface in order to achieve the required stoichiometry for the formation of an iron carbide, for example $\mathrm{Fe}_{3} \mathrm{C}$. It is derived from Equation 8.10 that only a variation of the ion fluence ratio $r_{\text {fluence }}$ can influence the layer period in such a way that larger $r_{\text {fluence }}$ lead to smaller layer periods.

\subsection{Comparison of the Multilayer Formation Models}

The model proposals described in this chapter and Chapter 6 partly take rather different approaches. $\mathrm{Wu}$ and Ting base their interpretation of the multilayer formation process on the assumptions that the deposition rates of carbon and the metal vary periodically due to a catalysis effect [1]. This model is therefore not applicable to MSIBD-grown films, where the deposition rates are determined by the respective ion currents and no reaction occurs. It is, however, successfull in explaining certain features of their films, such as the fact that the first layer at the interface to the substrate is always a metal-rich layer.

The model by He and co-workers is in principal an extended version of the model proposed by Corbella and co-workers [3, 2]. The assumptions are mainly based on non-equilibrium thermodynamics due to spinodal decomposition. Corbella and coworkers do not discuss the magnitude of diffusion and claim that their samples are kept at room temperature during deposition. The transport mechanism is not specified. Furthermore, it is not clarified whether the proposed affinity of one element to the substrate is thermodynamically in accordance with the element found near the substrate in experiments.

He and co-workers hold the incoming species responsible for an increase of diffusion in a region close to the surface. A moderate heating of the substrates can be expected due to the particle flux if no cooling is provided. Therefore, an enhanced 
diffusion may be a reasonable assumption. The additional transport induced by energetic particles is also taken into account. However, it is not clear from Reference [3] whether the resulting transport direction from the theoretical considerations is in agreement with the experimental findings.

The model ideas proposed in this chapter regard certain transport processes; particles impinge on the growing film, move around due to an ion-induced energy transfer to the material, and possibly leave the film due to sputtering. As described in Section 3.3, the difference in deposition energies when comparing MSIBD to R-MSD result in implantation depths of the incoming ions that differ only by a factor of 1.3-2. In R-MSD, incoming metal atoms from the target obtain smaller energies and are deposited into a region very close to the surface. Therefore, the considerations made above are not necessarily limited to the MSIBD technique but may also explain growth processes for samples grown by other techniques with similar deposition parameters, such as sputtering techniques or laser deposition. 


\section{Chapter 9}

\section{Magnetron Sputtered Films}

In order to investigate in more detail whether the multilayer formation model described in Chapter 8 is also applicable for deposition techniques other than MSIBD, a-C:Cu and a-C:Fe films were also grown by reactive magnetron sputtering deposition (R-MSD). The a-C:Cu system was chosen, because multilayers were already found by $\mathrm{Wu}$ and Ting for such films grown by magnetron sputtering. The a-C:Fe system was chosen as a carbide-forming example that was also found to show multilayers in MSIBD-grown films.

The films were grown on p-type $<100>$ silicon substrates. For each deposition, two substrates were mounted at equal distance to the centre of the sample holder in order to ensure equally thick films (the growth rate is not constant over the full range of the sample holder [155]). For the a-C:Fe films, the native $\mathrm{SiO}_{2}$ layer was removed prior to mounting the substrate in the vacuum chamber by etching in a $10 \%$ HF solution. The substrates were washed in destilled water and cleaned with acetone.

The sputter targets were a copper plate with a diameter of $76 \mathrm{~mm}$ and a thickness of $5 \mathrm{~mm}$ and a $99.5 \%$ pure iron target of the same diameter and $1 \mathrm{~mm}$ thickness. Before starting the deposition, the target was sputter cleaned by pure argon sputtering in order to remove any carbon that was deposited during the growth of the previous film as well as other precipitates from the residual gas. For film deposition, argon and methane gases were introduced into the chamber by two separate mass flow contollers with a total flow of $12 \mathrm{sccm}$. The pressure during deposition was $\sim 3 \times 10^{-4}$ mbar. 


\subsection{Magnetron Sputtered a-C:Cu}

In this section, some preliminary results on magnetron-sputtered a-C:Cu films are presented. In general, all films were grown at the same deposition parameters but at various $\mathrm{Ar} / \mathrm{CH}_{4}$ gas flow ratios. Only sample M001 was deposited at a higher target-substrate distance than the others. The precise deposition parameters are listed in Table 9.1 .

The RBS spectra of all the films are shown in Figure 9.1 [156]. All samples show a contamination with oxygen of up to 30 at.\%. This may be due to diffusion from the $\mathrm{SiO}_{2}$ layer at the substrate-film interface or oxygen incorporation during deposition from the residual gas in the deposition chamber. In order to rule out these two factors, further samples were pretreated by HF dipping, whereby the $\mathrm{SiO}_{2}$ layer is removed. Furthermore, the magnetron setup was connected to a nitrogen gas supply for venting. Therefore, the $\mathrm{H}_{2} \mathrm{O}$ content in the residual gas is reduced. Recent experiments that were not yet fully analyzed showed that oxygen-free a-C:Cu samples could successfully be grown.

The RBS spectra displayed in Figure 9.1 were normalized to the charge deposited during measurements. This allows a direct comparison of the copper signals for the relative copper content of the films. Samples M003 and M001 show the same copper concentration of about $50 \pm 5$ at.\%. The greater film thickness of M001, which can be seen from the shift of the silicon edge towards lower backscattering energies (indicated by small lines), is due to a longer deposition duration. The different target-substrate distance does not have an effect on the sample composition as this mainly influences the energy of the particles arriving at the substrate. Sample M004 was deposited at a smaller $\mathrm{Ar} / \mathrm{CH}_{4}$ ratio of 1.5/1. It shows a strong decrease of the copper signal from the substrate towards the surface. This effect has to be attributed

Table 9.1: Deposition parameters of the a-C:Cu films grown with R-MSD. All samples were deposited at room temperature onto p-Si $<100>$ substrates.

\begin{tabular}{cccc}
\hline \hline Sample No. & Ar $/ \mathrm{CH}_{4}$ ratio & Target-Substrate Distance $[\mathrm{cm}]$ & RF-power $[\mathrm{W}]$ \\
\hline M001 & $3 / 1$ & 5.5 & 100 \\
M002 & $1 / 1$ & 4 & 100 \\
M004 & $1.5 / 1$ & 4 & 100 \\
M003 & $3 / 1$ & 4 & 100 \\
\hline \hline
\end{tabular}




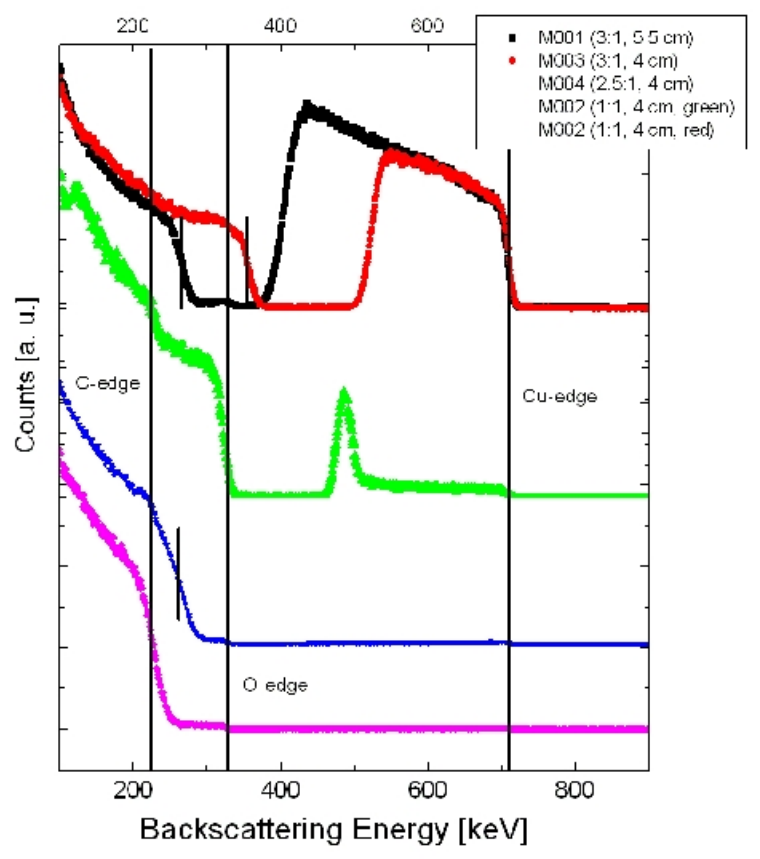

Figure 9.1: RBS spectra of the a$\mathrm{C}: \mathrm{Cu}$ samples.

to target poisoning: At the beginning of the deposition process, the copper target surface is clean and the full surface is available for the ablation of copper atoms. With ongoing deposition, carbon from the $\mathrm{Ar} / \mathrm{CH}_{4}$ plasma is deposited onto the target. Therefore, less copper atoms are sputtered off the target and the copper deposition rate decreases with respect to the carbon deposition rate. As carbon is then also sputtered off the target, a state of equilibrium is reached. The copper content near the target is as high as 20 at.\% and quickly decreases to below 2 at.\% towards the surface. In this spectrum, the oxygen surface edge and the shifted silicon edge appear at almost the same backscattering energy.

The two spectra of sample M002 (denoted as "red" and "green") were recorded at two different areas of the sample. This film is rather inhomogeneous in thickness which is evident from the rainbow-like colouring of the surface caused by interference. The two spectra were obtained from a red and a green area of the film. A RUMP analysis [133] yields a copper content of below 0.5 at.\% for both areas. The shifted silicon edge of the green area is indicated by a small line, in the spectrum of the red area, the shifted silicon edge is at the same position as the carbon surface edge. From these positions of the silicon edges it is found that the sample is thicker in the red area.

Obviously, the target poisoning effect and therefore the sample composition are very sensitive to the $\mathrm{Ar} / \mathrm{CH}_{4}$ gas flow ratio. This is understandable if we consider 
Figure 9.2: TEM micrograph of the a$\mathrm{C}: \mathrm{Cu}$ sample M001 $\left(\mathrm{Ar} / \mathrm{CH}_{4}=3 / 1\right.$, distance: $5.5 \mathrm{~cm})$. The film consists of clusters that are uniformly distributed throughout an amorphous carbon matrix. There is no indication of layers of varying concentration near the substrate or elsewhere in the film.

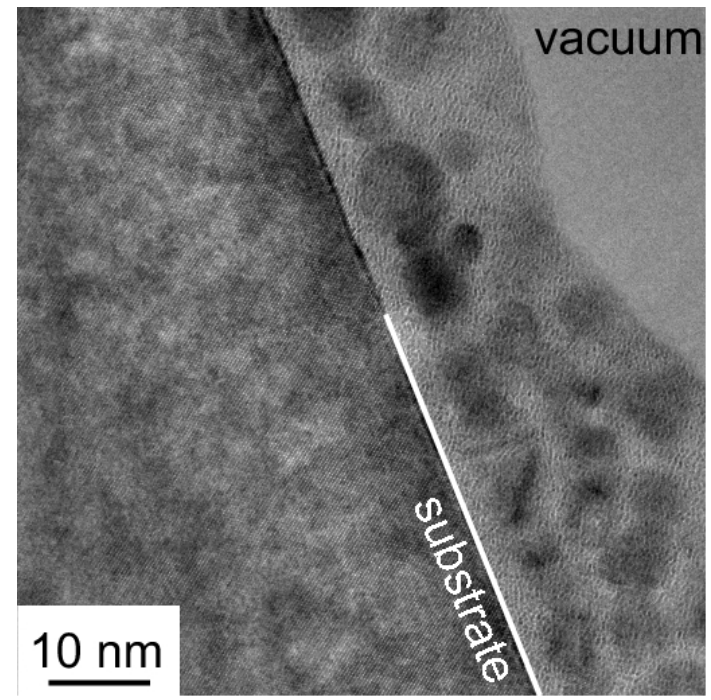

that the increase of the $\mathrm{CH}_{4}$ fraction results in two factors decreasing the sputtering of copper atoms from the target: There is less argon available for the sputtering of copper atoms and the copper target surface area is decreased due to the larger amount of carbon deposited on it. In addition to these two effects, there is more carbon available in the plasma for the deposition on the substrate. The large influence of the target poisoning effect on the sample composition is supported by the strong and sudden decrease of the copper signal in the RBS spectrum of sample M004.

Figure 9.2 shows a TEM micrograph of sample M001. This sample was deposited at an $\mathrm{Ar} / \mathrm{CH}_{4}$ ratio of $3 / 1$ and a target-substrate distance of $5.5 \mathrm{~cm}$. The specimen was too thick for high-resolution microscopy where the full film was intact. Only where part of the film had already been destroyed in the argon ion mill thinning process could HRTEM images be obtained. Therefore, Figure 9.2 shows only a small part of a film near the substrate. Copper clusters are uniformly distributed throughout an amorphous carbon matrix, but in this small section, an alignment of clusters parallel to the substrate can be seen. The clusters have a mean diameter of $5.2 \pm 0.7 \mathrm{~nm}$.

The EDX line scan across sample M001 displayed in Figure 9.3 shows three clear maxima in the copper signal. These maxima are about $100 \mathrm{~nm}$ apart and they cannot be correlated to any features in the overview STEM micrograph. This layer period is by 1-2 orders of magnitude larger than the ones found by $\mathrm{Wu}$ and Ting [1] or the layer distances described above for the MSIBD-grown a-C:Au and a-C:Fe films. The appearance of these layers is therefore attributed to a preparation artifact. 

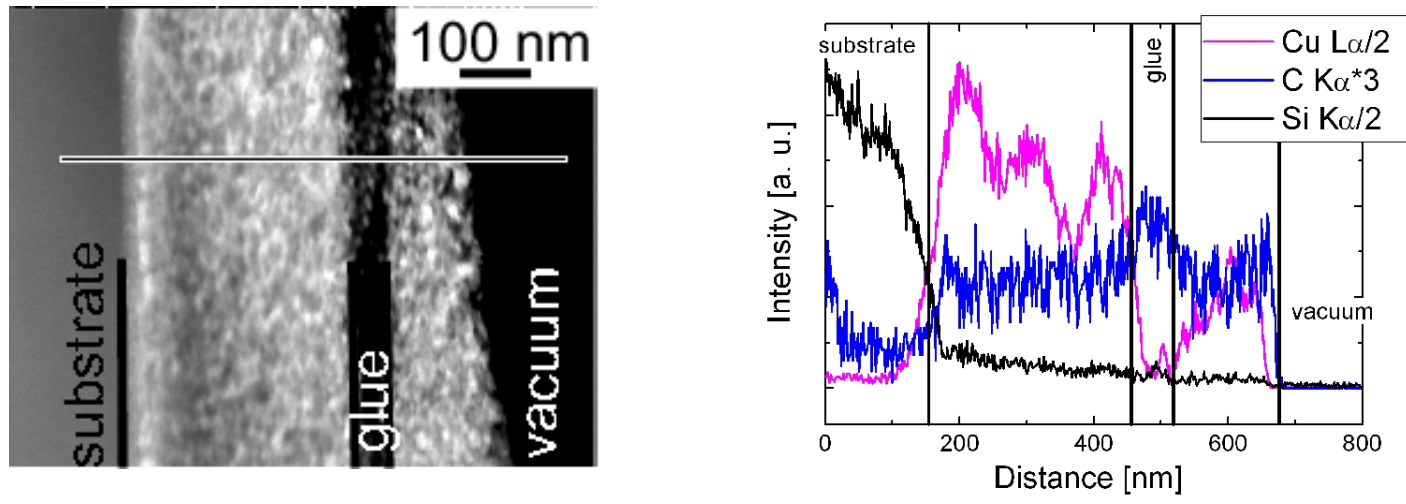

Figure 9.3: EDX scan of the a-C:Cu sample M001 along the line indicated in the micrograph. The three strong maxima in the copper signal cannot be correlated to areas of higher cluster density in the TEM micrographs.
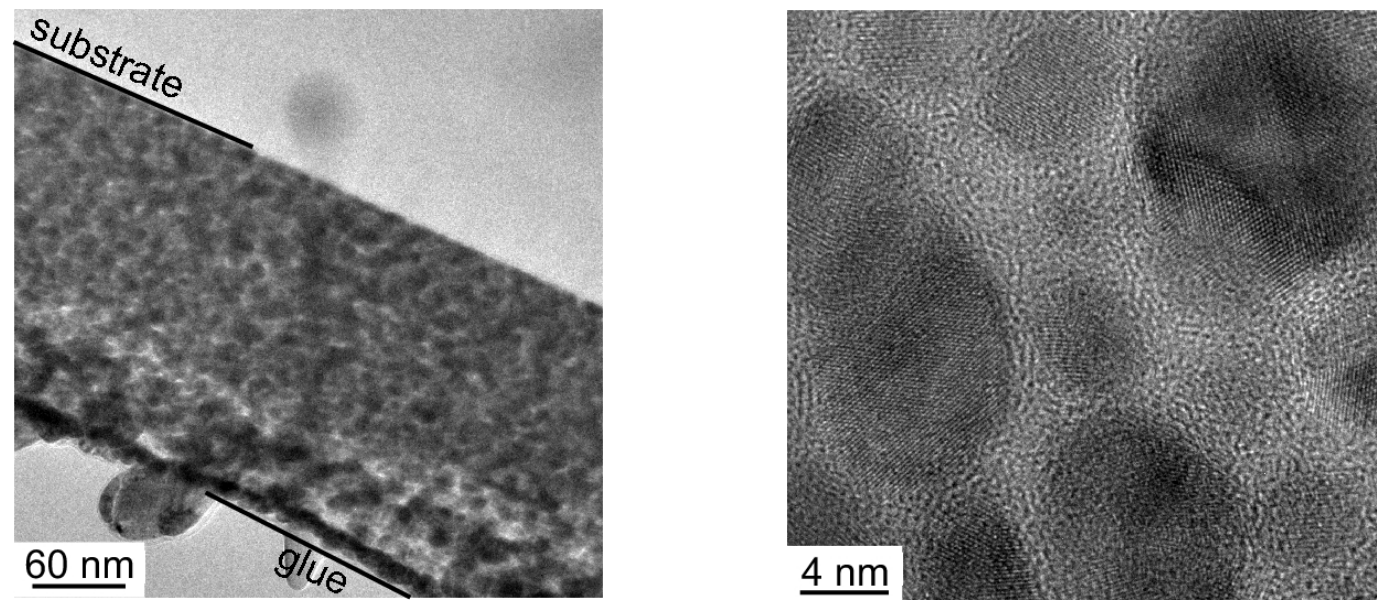

Figure 9.4: Overview TEM and HRTEM images of the a-C:Cu sample M003 $\left(\mathrm{Ar} / \mathrm{CH}_{4}=\right.$ $3 / 1$, distance: $4 \mathrm{~cm}$ ). Crystalline clusters are uniformly distributed throughout the amorphous carbon matrix. 

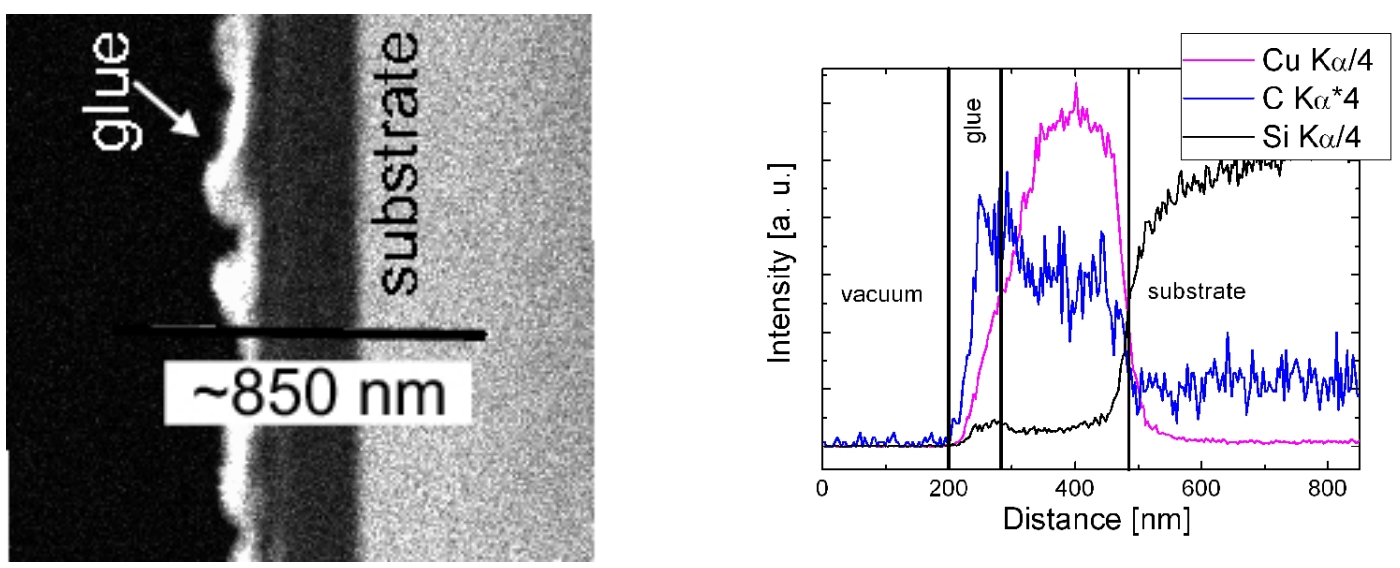

Figure 9.5: EDX scan of the a-C:Cu sample M003 along the line indicated in the micrograph. No periodic variation of the concentration is detectable.

Furthermore, an overall increase of the copper signal along with a decrease of the carbon signal towards the substrate is found, which can be explained by a moderate target poisoning. Usually, the intensities of the X-ray signals of all elements in the film decrease towards the film surface because of a wedge shape of the specimen generated by the thinning process.

Figure 9.4 shows an overview micrograph of sample M003. This film also consists of a uniform distribution of clusters, as it was seen for sample M001. In the appendant HRTEM image, clusters of various diameters can be distinguished. A mean cluster diameter of $8.0 \pm 2.7 \mathrm{~nm}$ is derived from TEM micrographs. The EDX line scan across sample M003 given in Figure 9.5 shows no periodic variations of the copper signal. However, the carbon signal decreases towards the substrate. As described above for sample M001, this can be explained by the target poisoning effect.

Sample M002 was also prepared for TEM analysis. This film has a purely amorphous structure, and no clusters could be found (not shown). This is not surprising, as it was found by RBS that this film contains only marginal amounts of copper.

\subsection{Magnetron Sputtered a-C:Fe}

Three sets of samples were grown: In the first series, the gas flow ratio was varied, while the target-substrate distance and the magnetron power were kept constant; in the second series, the target-substrate distance was varied at constant gas flow 
ratio and magnetron power; and for the third set, the magnetron power was varied at constant gas flow ratio and target-substrate distance. After connecting the magnetron deposition chamber to a nitrogen gas supply for venting and employing HF-dipping to the substrates prior to deposition, oxygen-free samples were obtained. The results are given in the following sections.

\subsubsection{Variation of the Gas Flow Ratio}

The variation of the gas flow ratio implies a variation of the amount of material sputtered off the target and the amount of carbon available for film deposition. Table 9.2 summarizes the deposition parameters for the samples grown with various $\mathrm{Ar} / \mathrm{CH}_{4}$ ratios. The samples M014 and M016 grown with $\mathrm{Ar} / \mathrm{CH}_{4}$ ratios of 5/1 and 15/1, respectively, were prepared for TEM analysis. The bulk of the films consist of crystalline clusters of nanometre size uniformly distributed throughout an amorphous carbon matrix (see Figures 9.6 and 9.7). The mean cluster diameters gained from the TEM micrographs are $2.3 \pm 0.5 \mathrm{~nm}$ and $3.7 \pm 0.7 \mathrm{~nm}$, respectively. Both samples show a layered structure near the substrate-film interface. In sample M014, the first layer is purely amorphous and rather bright. As the contrast depends, amongst other parameters, on the atomic weight of the material, this layer is probably iron-deficient. The next layer consists of a line of densely arranged clusters.

Table 9.2: Deposition parameters of the a-C:Fe films grown with R-MSD at various gas flow ratios. All samples were deposited at room temperature onto p-Si $<100>$ substrates.

\begin{tabular}{cccc}
\hline \hline Sample No. & Ar $/ \mathrm{CH}_{4}$ ratio & Target-Substrate Distance $[\mathrm{cm}]$ & RF power $[\mathrm{W}]$ \\
\hline M010 & $1 / 1$ & 4 & 100 \\
M008 & $1.5 / 1$ & 4 & 100 \\
M007 & $2 / 1$ & 4 & 100 \\
M009 & $3 / 1$ & 4 & 100 \\
M018 & $3 / 1$ & 4 & 100 \\
M011 & $3.5 / 1$ & 4 & 100 \\
M012 & $4 / 1$ & 4 & 100 \\
M014 & $5 / 1$ & 4 & 100 \\
M015 & $6 / 1$ & 4 & 100 \\
M013 & $7 / 1$ & 4 & 100 \\
M016 & $15 / 1$ & 4 & 100 \\
\hline \hline
\end{tabular}




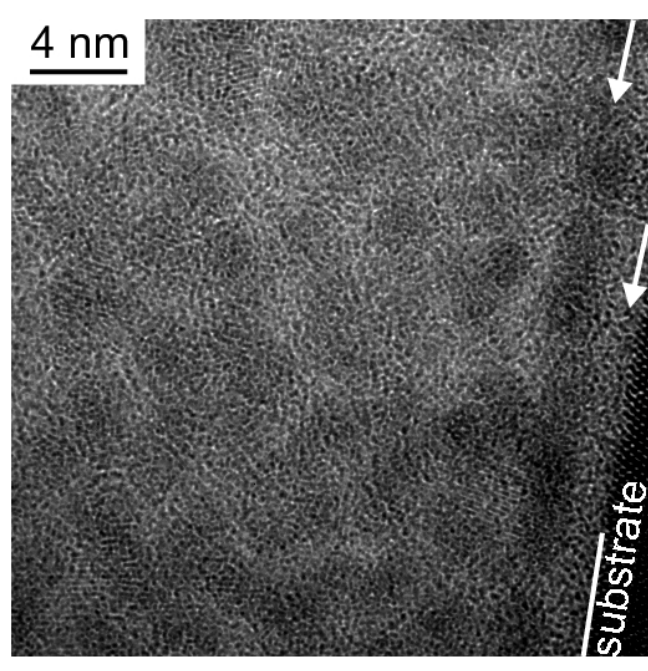

Figure 9.6: HRTEM micrograph of the a$\mathrm{C}: \mathrm{Fe}$ sample M014 (Ar/ $\left.\mathrm{CH}_{4}=5 / 1\right)$. The film consists of uniformly distributed clusters embedded in an amorphous carbon matrix. Close to the substrate, a clusterdeficient and a cluster-rich layer are indicated by two arrows.
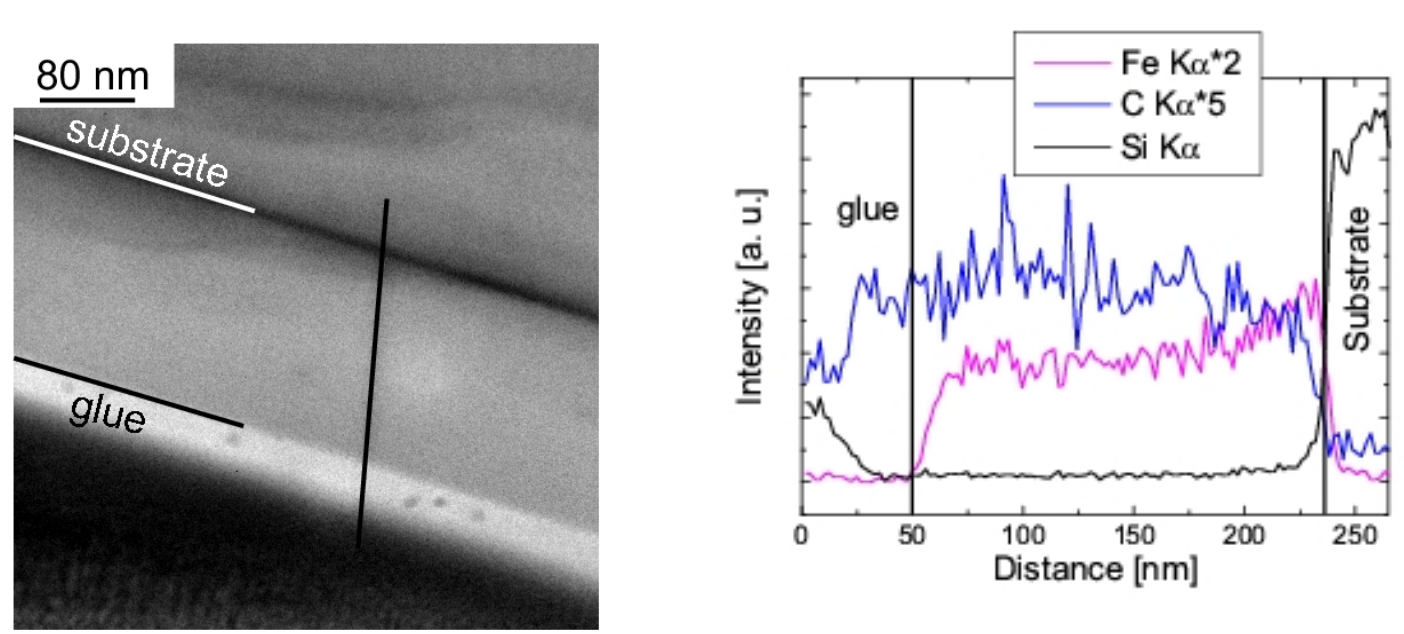

Figure 9.8: EDX scan of the a-C:Fe sample M014 along the line indicated in the micrograph. No variation of the iron concentration is detectable.

Figure 9.7: HRTEM micrograph of the a-C:Fe sample M016 $\left(\mathrm{Ar} / \mathrm{CH}_{4}=\right.$ 15/1). Four alternately cluster-deficient and cluster-rich layers near the substrate are indicated by arrows. Towards the surface, the layers become less pronounced. Otherwise, the film contains uniformly distributed crystalline clusters embedded in an amorphous carbon matrix. 

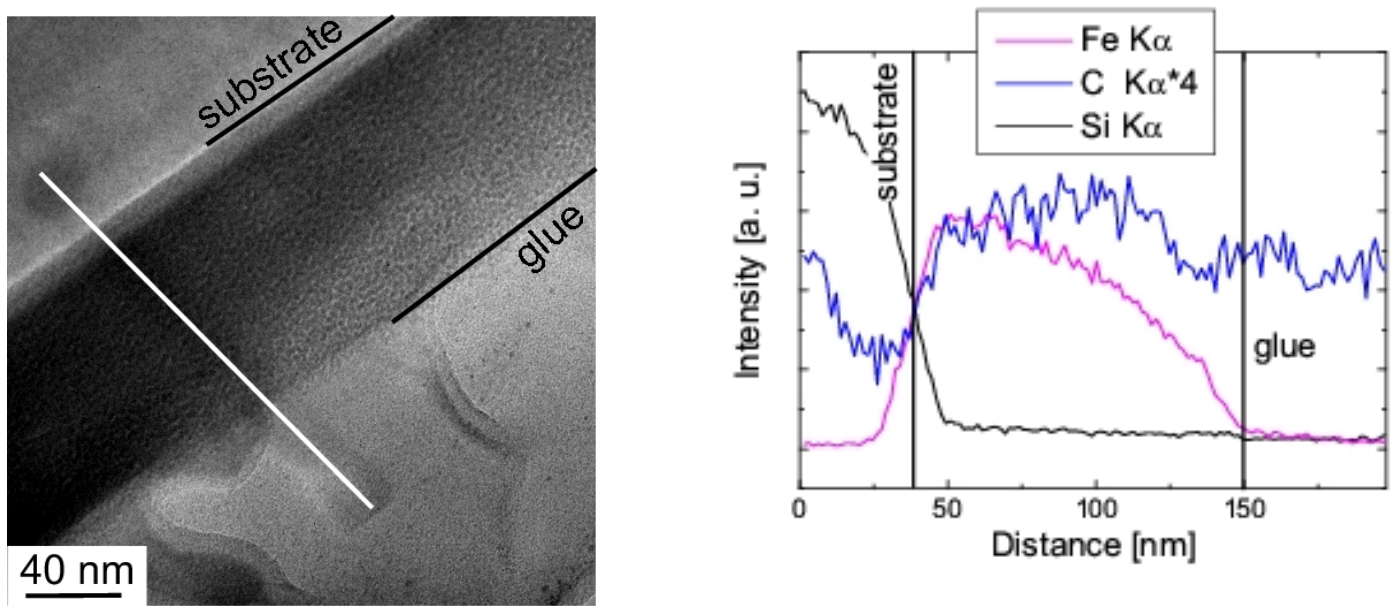

Figure 9.9: EDX scan of the a-C:Fe sample M016 along the line indicated in the micrograph. No variation of the iron concentration is detectable.

Sample M016 shows the same assembly plus an additional bright amorphous/dark cluster-containing bi-layer. The layers are indicated by arrows in Figures 9.6 and 9.7 .

EDX line scans were recorded across the films and are depicted in Figures 9.8 and 9.9. Neither the iron signals nor the carbon signals show periodic variations. The thicknesses of the layers visible in the HRTEM micrographs are of the order of $2-3 \mathrm{~nm}$. These small structures were not resolved in the line scans. Samples M007 through M012 were the first a-C:Fe films to be deposited with the R-MSD setup. The deposition times were rather short and the gas flow ratios small. The RBS spectra recorded of these samples are shown in Figure 9.10. For samples M007 through M010, the iron content is higher near the substrate. This property can be attributed to the target poisoning effect, as it was also found for the a-C:Cu films.

The signals were normalized to the value of the silicon edge in order to facilitate the comparison of the iron signals. From RUMP simulations [133], the iron concentration is found to range between $\sim 0.5$ and $\sim 8.5$ at.\% [156]. In general, the iron concentration in the films increases with the $\mathrm{Ar} / \mathrm{CH}_{4}$ ratio with exception of samples M011 and M012. These samples obtain smaller iron concentrations. A possible explanation is that these samples were grown after the deposition of sample M010, where the $\mathrm{Ar} / \mathrm{CH}_{4}$ ratio was $1 / 1$. The target may have been strongly contaminated with carbon at this low Ar content in the plasma. The pre-deposition sputter cleaning may not have been sufficient to remove all the carbon from the target. This 

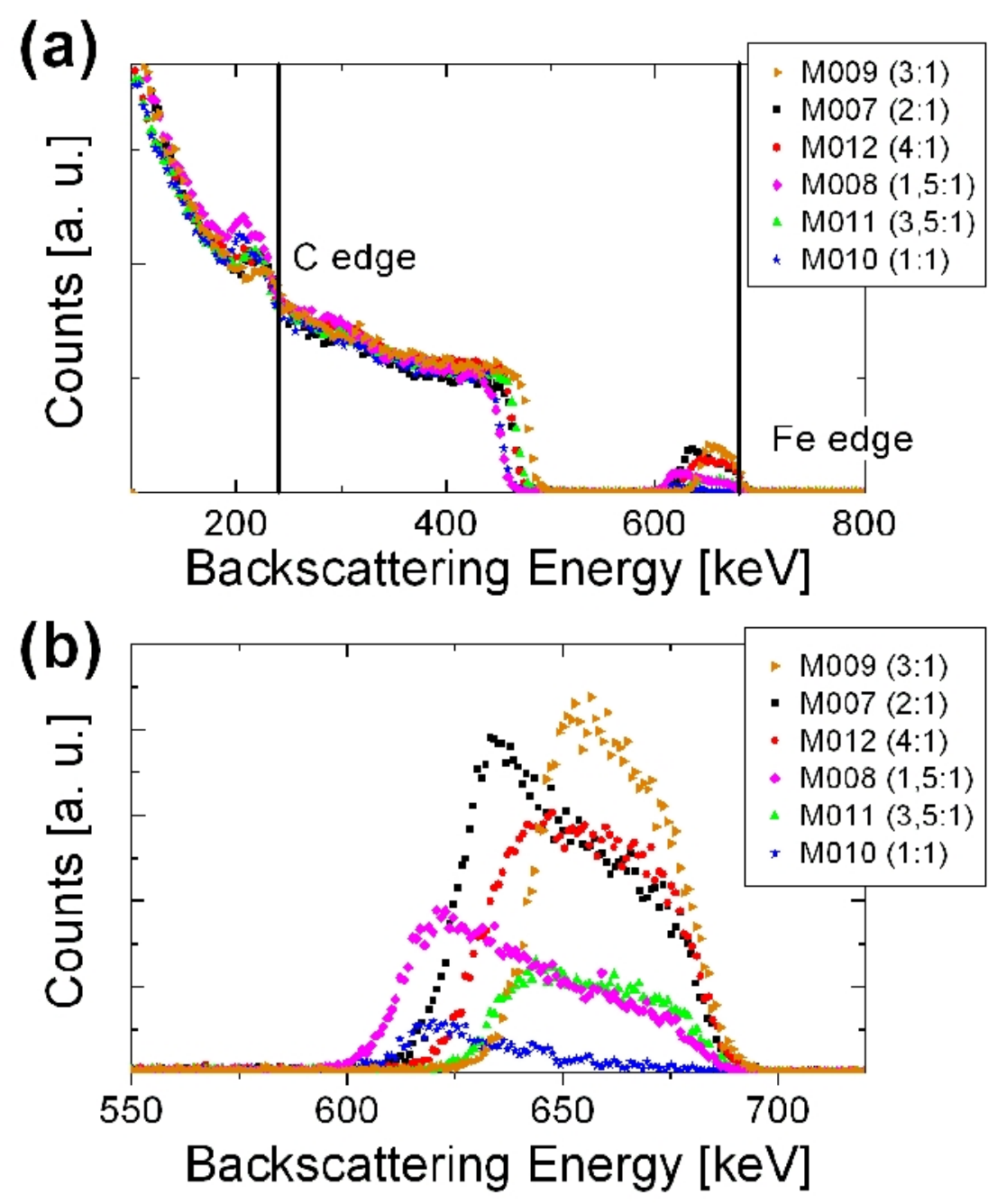

Figure 9.10: (a) RBS spectra of the first a-C:Fe samples grown with short deposition times and rather low gas flow ratios. (b) Enlarged display of the iron signal. The expected iron content dependency on the gas flow ratio is broken by samples M011 and M012. 

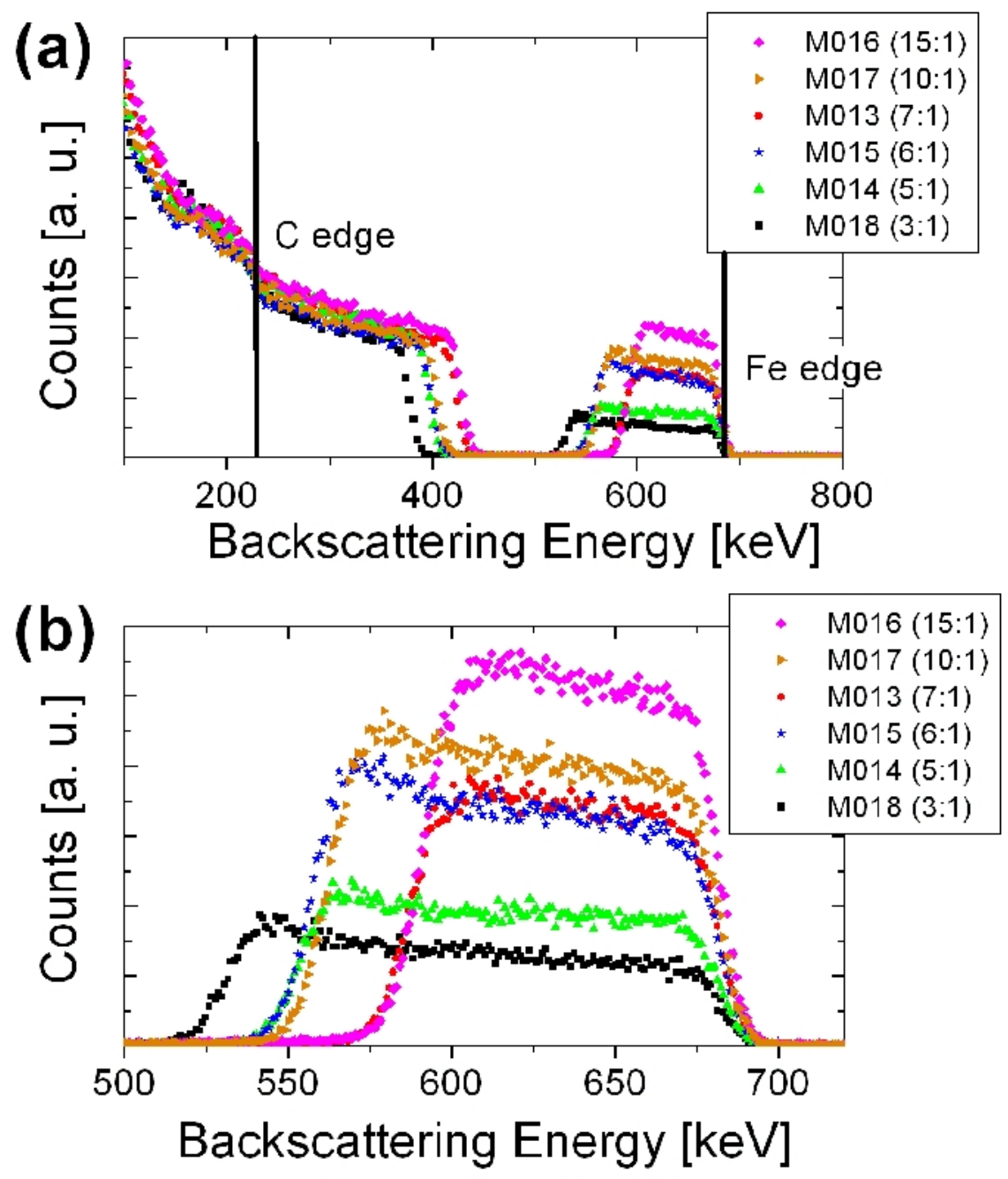

Figure 9.11: The RBS spectra of the a-C:Fe samples deposited with longer deposition times and higher gas flow ratios show the expected tendency of higher iron contents at higher gas flow ratios. 
Figure 9.12: RBS results of the a$\mathrm{C}:$ Fe films grown at various gas flow ratios. The dotted line is to guide the eye. The dependency of the iron concentration on the $\mathrm{Ar} / \mathrm{CH}_{4}$ ratio shows that larger amounts of argon in the gas mixture result in higher iron contents.

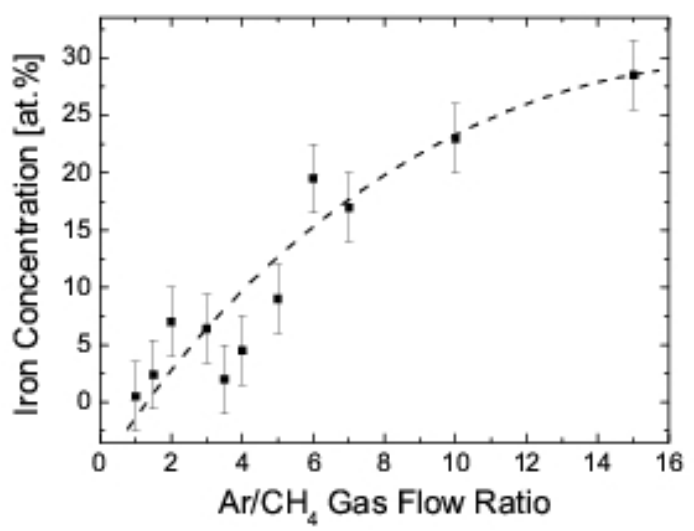

scenario is also supported by the fact that these two samples show a homogeneous iron concentration profile.

The RBS spectra of samples M013 through M018 are shown in Figure 9.11. Again, the spectra are normalized to the value of the silicon edge for better comparison. The raised iron signal at lower energies corresponding to a higher iron content is also present in these spectra. In this case, the iron concentration increases with the $\mathrm{Ar} / \mathrm{CH}_{4}$ ratio from $\sim 6.4$ to $\sim 28.5$ at.\% [156]. This result is in agreement with the expectation: For smaller $\mathrm{Ar} / \mathrm{CH}_{4}$ ratios, the available amount of carbon is larger and less iron is sputtered off the target. The iron concentrations of all samples are displayed in Figure 9.12 in dependency of the gas flow ratio. In this graph, the general increase of the iron concentration with the $\mathrm{Ar} / \mathrm{CH}_{4}$ ratio is evident and a possible saturation of the iron concentration is suggested by the development of the values.

The concentration variation suggested by the HRTEM micrographs of Figures 9.6 and 9.7 are by far too small to be resolved by RBS. Probably, this variation is a result of reaching a steady state of deposition at the beginning of the deposition process.

No reliable information can be gained on the cluster size dependency of the gas flow ratio, as mean cluster diameters could only be measured for the two samples M014 and M016. However, the trend shows that smaller iron concentrations result in smaller clusters. 


\subsubsection{Variation of the Target-Substrate Distance}

The distance between the target and the substrate has substantial influence on the energy of the particles impinging on the film. At smaller distances, the particles collide less often with other particles and therefore lose less energy. This may have a distinct influence in the film morphology. The deposition parameters for this set of samples are listed in Table 9.3 .

Figures 9.13, 9.14, and 9.15 show the HRTEM micrographs of the samples M019 $(3.5 \mathrm{~cm})$, M020 $(5 \mathrm{~cm})$, and M023 $(7 \mathrm{~cm})$, respectively. Figure 9.7 can be taken for comparison, because sample M016 was deposited with the same parameters as the samples of this series at a target-substrate distance of $4 \mathrm{~cm}$. The cluster sizes derived from HRTEM are as follows: $3.5 \pm 0.6 \mathrm{~nm}$ at $3.5 \mathrm{~cm}, 3.7 \pm 0.7 \mathrm{~nm}$ at $4 \mathrm{~cm}$,

Table 9.3: Deposition parameters of the a-C:Fe films grown with R-MSD at various target-substrate distances. All samples were deposited at room temperature onto p-Si $<100>$ substrates.

\begin{tabular}{cccc}
\hline \hline Sample No. & Ar $/ \mathrm{CH}_{4}$ ratio & Target-Substrate Distance $[\mathrm{cm}]$ & RF power $[\mathrm{W}]$ \\
\hline M019 & $15 / 1$ & 3.5 & 100 \\
M016 & $15 / 1$ & 4 & 100 \\
M022 & $15 / 1$ & 4.5 & 100 \\
M020 & $15 / 1$ & 5 & 100 \\
M021 & $15 / 1$ & 6 & 100 \\
M023 & $15 / 1$ & 7 & 100 \\
\hline \hline
\end{tabular}

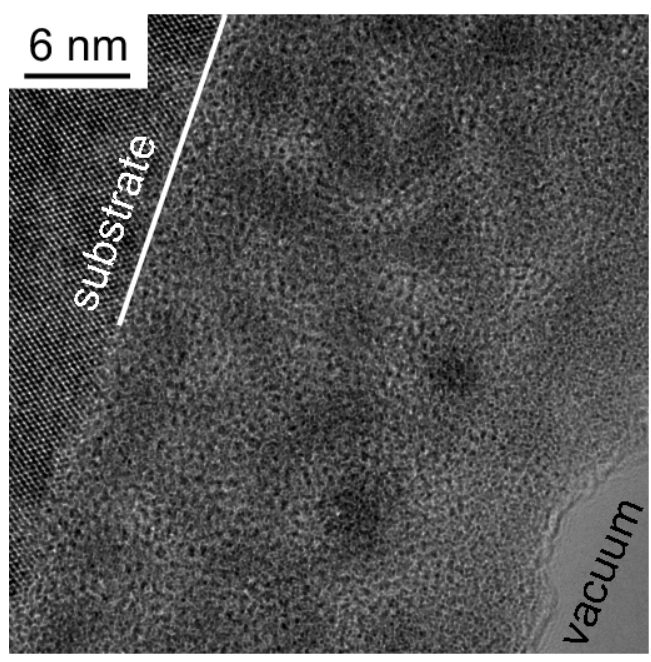

Figure 9.13: HRTEM micrograph of the a-C:Fe sample M019 (distance: $3.5 \mathrm{~cm}$ ). The film consists of clusters that are uniformly distributed throughout an amorphous carbon matrix. There is no indication of layers of varying concentration near the substrate or elsewhere in the film. 


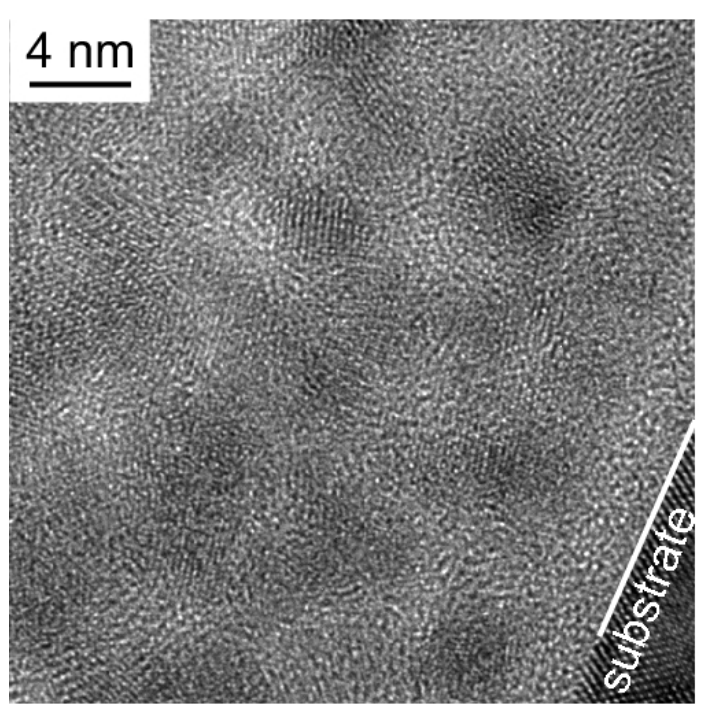

Figure 9.14: HRTEM micrograph of the a-C:Fe sample M020 (distance: $5 \mathrm{~cm}$ ). Clusters in this sample are uniformly distributed and no layer structure is visible.

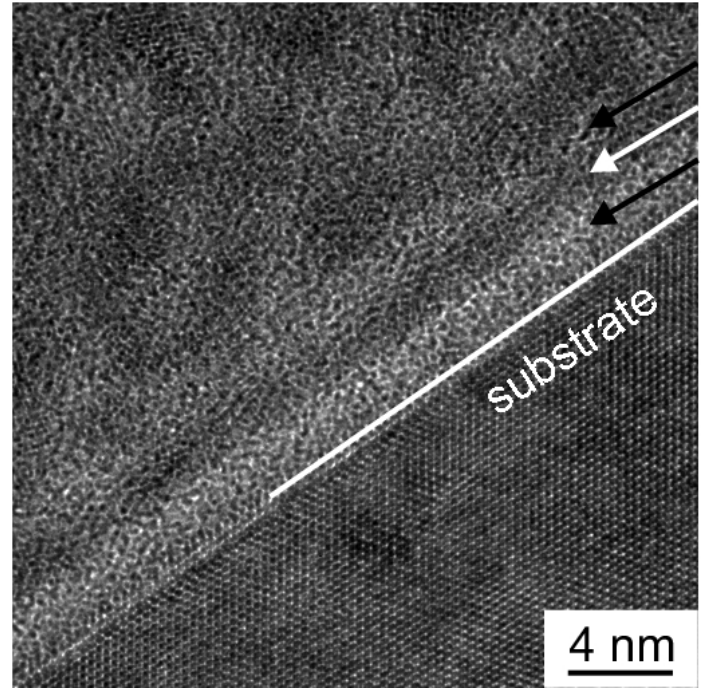

Figure 9.15: HRTEM micrograph of the a-C:Fe sample M023 (distance: $7 \mathrm{~cm}$ ). This sample shows three alternately cluster-deficient and cluster-rich layers. Towards the surface, the film contains a uniform distribution of clusters within an amorphous carbon matrix.

$3.2 \pm 0.7 \mathrm{~nm}$ at $5 \mathrm{~cm}$, and $3.0 \pm 0.5 \mathrm{~nm}$ at $7 \mathrm{~cm}$ target-substrate distance. Within the interval of error, the cluster size does not show any dependency on the targetsubstrate distance.

In addition to sample M016 (4 cm-discussed in the previous section), only the micrograph of sample M023 $(7 \mathrm{~cm})$ in Figure 9.15 shows three alternately bright and dark layers. Obviously, this phenomenon is not directly dependent on one of the measurable parameters.

The EDX linescans of samples M020 and M023 are displayed in Figures 9.16 and 9.17. Neither of them shows a periodic variation of the iron concentration. The decrease of the iron and carbon signals in Figure 9.17 represents the wedge shape of the specimen due to the ion mill thinning process.

Figure 9.18 shows the RBS spectra of the target-substrate distance series normalized to the silicon edge [156]. The iron concentration does not change with the target-substrate distance up to a distance of $7 \mathrm{~cm}$. The concentration of samples M016, M019, and M020 through M022 remains at very similar values around 

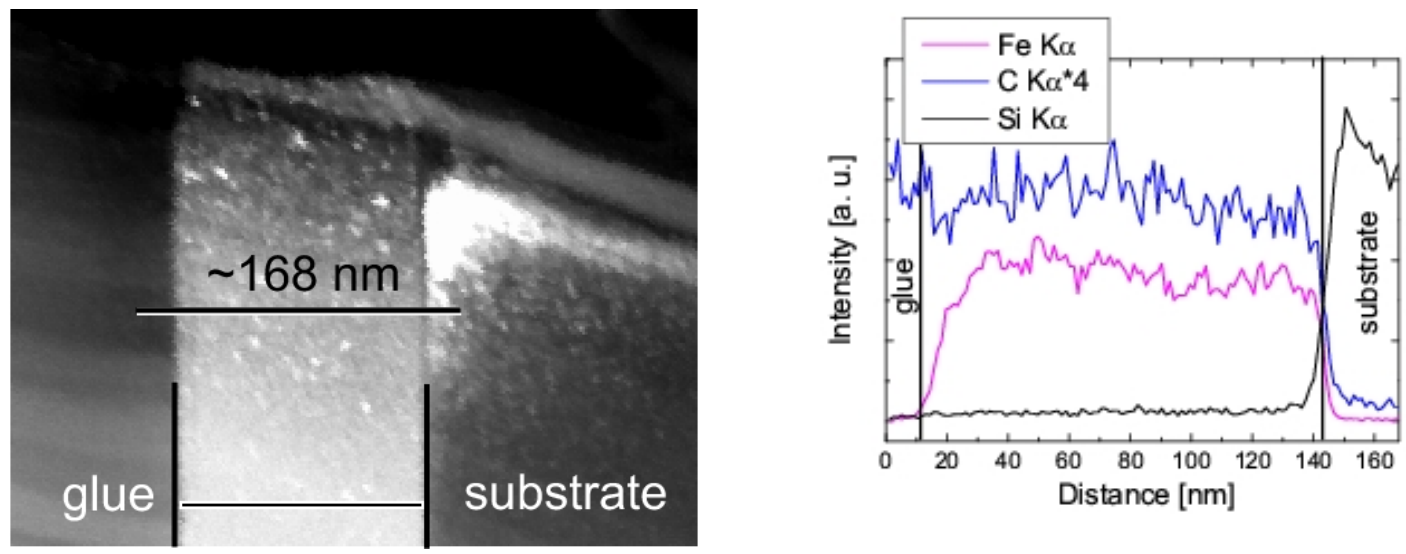

Figure 9.16: EDX scan of the a-C:Fe sample M020 along the line indicated in the micrograph. No concentration variation is detectable.
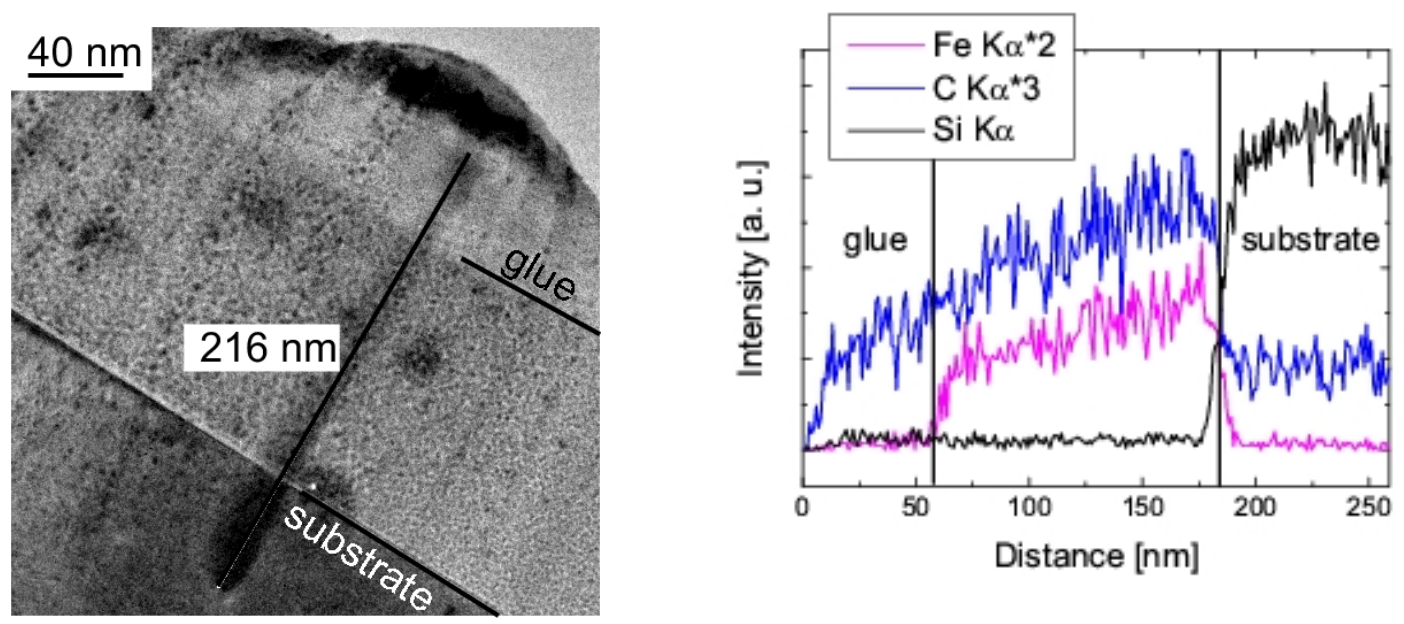

Figure 9.17: EDX scan of the a-C:Fe sample M023 along the line indicated in the micrograph. No concentration variation is detectable. 

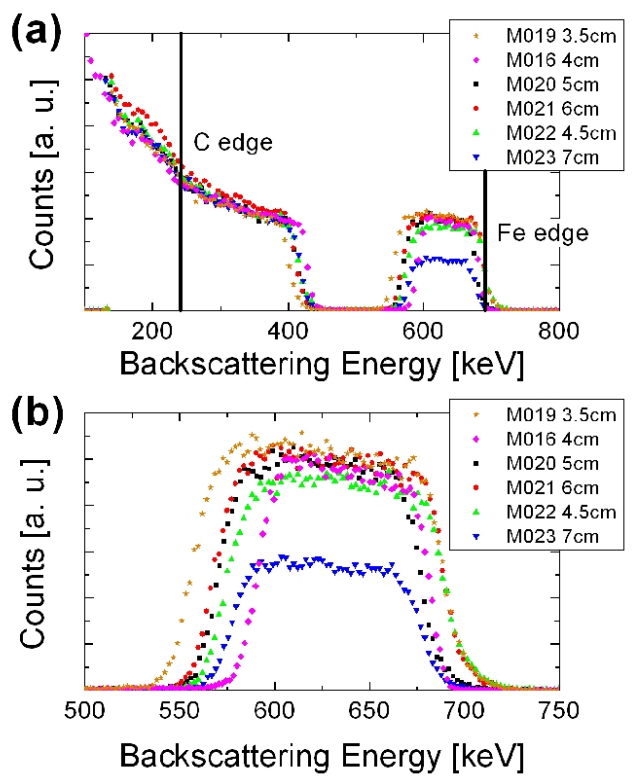

Figure 9.18: (a) RBS spectra of the samples grown at various target-substrate distances. (b) Enlargement of the iron signal. The iron content does only decrease at a distance of $7 \mathrm{~cm}$.

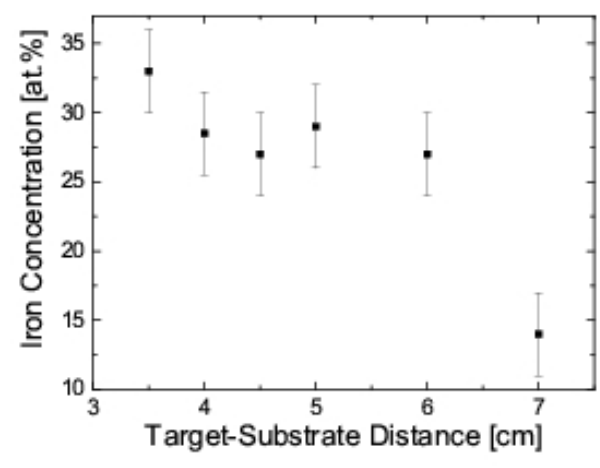

Figure 9.19: RBS results of the a-C:Fe films grown at various targetsubstrate distances. The iron concentration shows no clear dependency on the target-substrate distance but drops abruptly when the distance is raised from 6 to $7 \mathrm{~cm}$.

$\sim 30$ at.\%, while sample M023 contains only 14 at.\% iron. This becomes even more clear from Figure 9.19. The concentration shows no monotonous dependency on the target-substrate distance.

If now the results of the TEM analysis are considered together with the RBS results, the following is found: The cluster sizes vary only very little, as it is the case for the iron concentration. Sample M023 is the exception from this rule. This sample contains less iron and contains the smallest clusters. However, such small changes in the target-substrate distance should not have a considerable influence on the film composition. It is therefore questionable whether increasing the targetsubstrate distance from $6 \mathrm{~cm}$ to $7 \mathrm{~cm}$ is responsible for the sudden decrease of the iron concentration. 


\subsubsection{Variation of RF Power}

For the last set of samples, the RF power was varied. This parameter influences the sputtering yield of the target material by the ions from the plasma and the energy the sputtered atoms. In this manner, it may have an influence on both the sample composition and morphology.

Table 9.4: Deposition parameters of the a-C:Fe films grown with R-MSD at various RF powers. All samples were deposited at room temperature onto p-Si $<100>$ substrates.

\begin{tabular}{cccc}
\hline \hline Sample No. & Ar $/ \mathrm{CH}_{4}$ ratio & Target-Substrate Distance $[\mathrm{cm}]$ & RF power $[\mathrm{W}]$ \\
\hline M027 & $15 / 1$ & 4 & 80 \\
M025 & $15 / 1$ & 4 & 90 \\
M016 & $15 / 1$ & 4 & 100 \\
M024 & $15 / 1$ & 4 & 110 \\
M026 & $15 / 1$ & 4 & 120 \\
\hline \hline
\end{tabular}
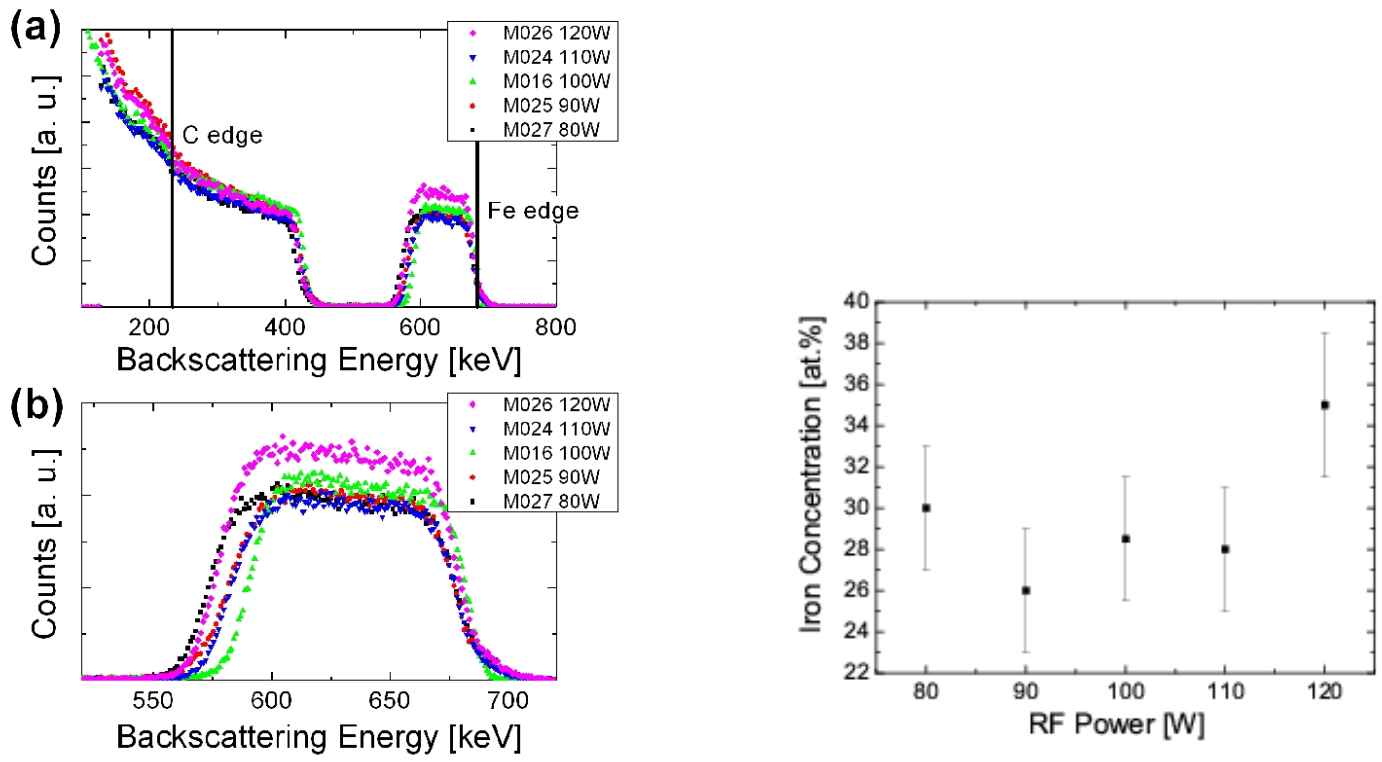

Figure 9.20: (a) RBS spectra of the a-

Figure 9.21: RBS results of the a-C:Fe $\mathrm{C}:$ Fe samples grown with various RF powers. (b) Enlargement of the iron signal. The iron concentration is only increased for an RF power of $120 \mathrm{~W}$. films grown at various magnetron powers. The iron concentration shows no clear dependency on the target-substrate distance. 
Figure 9.20 shows the RBS spectra of these samples [156]. Again, the spectra are normalized to the silicon edge for better comparability of the iron signals. The iron concentration is similar for all samples, with exception of sample M026, which was grown with the highest RF power of $120 \mathrm{~W}$. This sample contains the slightly higher amount of $\sim 35 \%$ iron. Such an increase is reasonable, as the increase of the RF power results in higher sputtering yields. Therefore, more iron atoms are sputtered off the target and transported to the substrate. Up to now, non of the samples could successfully be prepared for TEM analysis. Therefore, no information on the morphology is available yet.

\subsection{Summary and Discussion}

Copper- and iron-containing amorphous carbon films were grown using R-MSD in order to investigate a possible multilayer formation. While a-C: $\mathrm{Cu}$ films grown by MSIBD consist of uniformly distributed clusters of nanometre size within an amorphous carbon matrix, $\mathrm{Wu}$ and Ting found a periodic concentration variation for their magnetron-sputtered a-C:Cu films [1]. Although the deposition parameters were chosen very similar, such a film morphology could not be reproduced by R-MSD in this work. However, there are a number of differences between the setups: First of all, the films reported on by Wu and Ting were grown using a DC R-MSD setup, while the setup used here is driven by an RF power supply. Therefore, the energy distribution of the particles impinging on the substrate are possibly quite different even though the nominal power was $100 \mathrm{~W}$ in both cases. During recent discussions, $\mathrm{Ms}$. Wu reported on the formation of multilayers in a-C:Pt films grown by RF RMSD at higher RF powers [157]. Furthermore, in the magnetron setup used by Wu and Ting, the target and substrate holder are positioned at an inclined angle towards each other. In the setup used for this thesis, the target and substrate surfaces are parallel to each other. Therefore, the particles impinge on the substrate at different angles in both studies. This may result in slightly different ion ranges within the films. The target holder of Wu's and Ting's setup is not water cooled. This may also have an influence on the mobility of the deposited species. Furthermore, the a$\mathrm{C}: \mathrm{Cu}$ samples reported on above are severely oxygen contaminated. The formation of copper oxides is very likely to have a strong influence of the morphology of the films.

The EDX line scan of sample M001 suggested a periodic variation of the copper 
concentration throughout the film. However the distance of the maxima is $1-2$ orders of magnitude larger than the layer distances found for the MSIBD-grown a-C:Au and a-C:Fe films. A variation of this magnitude should have been resolved by RBS. As the copper signal in the respective RBS spectrum shows no such periodic variation, the maxima in the EDX line scan are obviously a preparation artifact.

The cluster diameter of the R-MSD deposited a-C: $\mathrm{Cu}$ films are of the same order of magnitude as for the MSIBD films. While films M001 and M003 were deposited at the same gas flow ratios and exhibit the same copper concentration, their mean cluster diameters are quite different. The clusters of sample M001, which was deposited at a larger target-substrate distance, are smaller. Presumably, the particles impinging on the substrate have smaller energies and therefore the mobility of the copper particles is smaller. The composition of the samples shows the expected dependence on the gas flow ratio.

Corbella and co-workers observed a multilayer formation also for a-C films containing the carbide-forming metal titanium 2]. Furthermore, MSIBD-grown a-C:Fe films also show a multilayer structure. Therefore, the morphology of a-C:Fe films grown by R-MSD was also of interest. As in the case of the a-C:Cu films, no multilayer structure was found. The exception is a variation over two to four layers near the substrate for some films. As no correlation with the measurable parameters can be found, this effect is not in accordance with the model proposed by Corbella and co-workers, who also find a diminishing multilayer structure of their films. It rather has to be attributed to variations of the deposition conditions at the beginning of the process, when target poisoning and carbon sputtering of the target are not yet in equilibrium. Again, a comparison of the results is rather difficult due to the differences in the experimental setups. The R-MSD setup used by Corbella and coworkers is driven by a pulsed DC power supply and the substrates were RF-biased yielding a different energy distribution of the particles impinging on the substrate. Furthermore, a comparison is difficult, as Corbella and co-workers found multilayers for the non-magnetic metal titanium. Especially in magnetron sputter deposition, the deposition conditions can be very different when the target material is magnetic.

The compositions of the a-C:Fe films investigated here depend mainly on the $\mathrm{Ar} / \mathrm{CH}_{4}$ gas flow ratio. The composition changes only at the largest target-substrate distance and the largest $\mathrm{RF}$ power to smaller or higher iron concentrations, respectively. However, the strong change in composition when changing the targetsubstrate distance from $6 \mathrm{~cm}$ to $7 \mathrm{~cm}$ remains unclear. Generally, much higher 
$\mathrm{Ar} / \mathrm{CH}_{4}$ ratios have to be used in order to obtain films with a reasonable metal concentration than in the case of a-C:Cu films. This arises from the magnetic nature of the iron target, which leads to a magnetic short circuit and the enhancement of the permanent magnet on plasma is significantly reduced.

The cluster diameters of the a-C:Fe films are slightly smaller for the a-C:Fe films than for the a-C:Cu films as it was also the case for the MSIBD films. The tendency of smaller clusters at smaller metal concentrations can also be found for these samples. Furthermore, the cluster diameters for all samples deposited at different target-substrate distances are in the range of 3.0 and $3.7 \mathrm{~nm}$. In particular, the mean cluster size remains at $3.5 \mathrm{~nm}$ for sample M019, which was closest to the target during deposition, although the iron concentration was notably reduced for this sample. This also implies a higher mobility of iron and carbon atoms in the sample at shorter target-substrate distances. 


\section{Chapter 10}

\section{Final Discussion and Conclusion}

The growth of metal-containing amorphous carbon films by MSIBD results in an interesting variety of different structures, depending on the deposition parameters and the metal used. A uniform distribution of metal clusters throughout a matrix, as it is widely reported on in the literature (see Section 1.2), was established for a-C:Cu films at a low copper ion energy of $40 \mathrm{eV}$ as well as for a-C:Au films at high carbon and gold ion energies of $200 \mathrm{eV}$.

Furthermore, silver atoms were found to segregate at the film surface during MSIBD. Only if the carbon/silver ion fluence ratio is not too high and therefore the sputtering yield smaller than one, can silver accumulate at the film surface. The processes driving this behaviour were discussed in Section 8.2. In this context, the intriguing evolution of the multilayer structure found for MSIBD-grown a-C:Au films was also treated. The self-organized formation of metal-rich and metal-deficient multilayers during the co-deposition of carbon and metal ions can be modeled as an interplay of the following: (a) an ion triggered atomic transport, (b) the segregation of metal atoms at the surface, (c) preferential sputtering, and (d) the stability of small metal clusters under ion impact. This phenomenon was observed and discussed for the first time in the course of this work and, at nearly the same time, similar morphologies were reported on by $\mathrm{Wu}$ and Ting [1].

The deposition of carbon and the carbide-forming metal iron results in a very similar layer structure. However, from the considerations of Section 8.3 and the experimental results given in Section 7.3 , it becomes evident that sputtering plays only a minor role in this case and chemical properties are of importance.

Multilayer morphologies of a-C:Me films as well as AuNi films have already been reported in the literature, as was discussed in Chapter 6, and various models were 
proposed [1, 2, 3]. It was shown in Section 8.8 that these models have only limited success in explaining the formation of multilayers by MSIBD. Part of the mechanisms considered crucial do not occur during MSIBD, as chemical reactions or thermal diffusion.

The multilayers reported on in the literature and in this thesis are all features on the nanometre scale with very similar morphologies. The metal-rich layers always consist of metallic particles, which are usually of crystalline structure. The layers are never fully closed metal layers. Nevertheless, there are some differences: The multilayers found by $\mathrm{Wu}$ and Ting usually start with a metal-rich layer near the substrate [1]. This is the opposite for the multilayers presented in this work. Furthermore, the multilayers found by Corbella and co-workers diminish towards the surface [2].

While the mechanisms proposed in Chapter 8 may account for a multilayer formation also in the deposition techniques used by Wu and Ting, Corbella and coworkers, and He and co-workers, there are still some experimental discrepancies. For example, the effect that the first layer near the substrate is always a metal layer in the films reported on by $\mathrm{Wu}$ and Ting [1] cannot be explained by the model proposed in Chapter 8 for non-carbide forming metals with vanishing solubility in carbon. Neither does it account for the disappearance of the multilayer structure, as it was seen by Corbella and co-workers [2].

The community of researchers working on a-C:Me films grown by techniques that employ hyperthermal species is rather large. Nevertheless, only few reported on a multilayer morphology of their films. This can be mainly attributed to two factors: First, the methods implemented are often not suitable for the analysis of the vertical structure of the sample. When TEM is performed, the samples are often not prepared for cross-sectional microscopy but rather plane view images are recorded. The second and more important reason is probably that the deposition conditions for multilayer formation are not easily met. The deposition parameters at which multilayers will form even depend quite sensitively on the geometry of the deposition setup. Therefore, no multilayers were observed in the R-MSD-grown films of this work.

It has been shown that there are several interesting and promising model approaches for the formation of the various morphologies found in films grown by the deposition of hyperthermal species. However, non of the proposed models has the capability to explain all of the features of the various films. In the past, MSIBD 
has proved to be a suitable tool for a precise investigation of film characteristics in dependence on various growth parameters, as these can be precisely and independently varied. In this context, it is more promising to work with ion sources that offer a narrow ion energy distribution. Furthermore, it would be interesting to thoroughly characterize the deposition conditions of the respective setups of the other deposition techniques. Most importantly, the energy of the particles impinging on the substrate should be determined as a function of all parameters that may have an influence. The knowledge of these parameters is essential in understanding the evolution of the various structures found for the different samples, techniques, and setups. In this context, it should then be possible to verify the predicted influence of the deposition parameters on the evolution of the different structures and their respective quantitative characteristics, such as cluster sizes and layer periods.

He and co-workers recently reported on a multilayer structure of $\mathrm{AuNi}$ films [3]. The effect is therefore obviously not limited to carbon-metal systems. It is an exciting challenge to see if a multilayer film morphology can also be obtained for other systems meeting certain demands, such as the immiscibility of two phases and appropriate sputtering yields. 


\section{References}

[1] W.-Y. Wu and J.-M. Ting, Self-assembled alternating nano-scale layers of carbon and metal, Chem. Phys. Lett. 388, 312 (2004).

[2] C. Corbella, B. Echebarria, L. Ramírez-Piscina, E. Pascual, J. L. Andújar, and E. Bertran, Spontaneous formation of nanometric multilayers of metal-carbon films by up-hill diffusion during growth, Appl. Phys. Lett. 87, 213117 (2005).

[3] J. H. He, C. A. Carosella, G. K. Hubler, S. B. Qadri, and J. A. Sprague, Bombardment-induced tunable superlattices in the growth of Au-Ni films, Phys. Rev. Lett. 96, 056105 (2006).

[4] U. Kreibig and M. Vollmer, Optical properties of metal clusters, Springer, New York (1995).

[5] J. P. Zhao, Z. Y. Chen. M. Lu, and J. W. Rabalais, Linear optical properties of a Ti-SiO $\mathrm{O}_{2}$ nanoparticle composite, J. Appl. Phys. 93, 566 (2003).

[6] L. Serra and A. Rubio, Core polarization in the optical response of metal clusters: Generalized time-dependent density-functional theory, Phys. Rev. Lett. 78, 1428 (1997).

[7] J. Lermé, B. Palpant, B. Prével, M. Pellarin, M. Treilleux, J. L. Vialle, A. Perez, and M. Broyer, Quenching of the size effects in free and matrixembedded silver clusters, Phys. Rev. Lett. 80, 5105 (1998).

[8] I. Tanahashi, Y. Manabe, T. Tohda, S. Sasaki, and A. Nakamura, Optical nonlinearities of $\mathrm{Au} / \mathrm{SiO}_{2}$ composite thin films prepared by a sputtering method, J. Appl. Phys. 79, 1244 (1996).

[9] H. B. Liao, R. F. Xiao, J. S. Fu, P. Yu, G. K. L. Wong, and P. Sheng, Large third-order optical nonlinearity in $\mathrm{Au}: \mathrm{SiO}_{2}$ composite films near the percolation threshold, Appl. Phys. Lett. 70, 1 (1997).

[10] H. Inouye, K. Tanaka, I. Tanahashi, Y. Kondo, and K. Hirao, Mechanism of a terahertz optical Kerr shutter with a gold nanoparticle system, J. Phys. Soc. Jpn. 68, 3810 (1999). 
[11] N. Del Fatti and F. Vallée, Ultrafast optical nonlinear properties of metal nanoparticles, Appl. Phys. B 73, 383 (2001).

[12] K. Koski, J. Hölsä, J. Ernoult, and A. Rouzaud, The connection betweeen sputter cleaning and adhesion of thin solid films, Suf. Coat. Technol. 80, 195 (1996).

[13] M. M. Morshed, B. P. McNamara, D. C. Cameron, and M. S. J. Hashmi, Effect of surface treatment on the adhesion of DLC film on 316L stainless steel, Surf. Coat. Technol. 163-164, 541 (2003).

[14] I. Gerhards, C. Ronning, H. Hofsäss, M. Seibt, and H. Gibhardt, Ion beam synthesis of diamond-like carbon thin films containing copper nanocrystals, J. Appl. Phys. 93, 1203 (2003).

[15] S. Zhang, X. L. Bui, X. T. Zeng, and X. Li, Towards high adherent and tough a-C coatings, Thin Sol. Films 482, 138 (2005).

[16] R. J. Narayan, Pulsed laser deposition of functionally gradient diamondlike carbon-metal nanocomposites, Diamond Relat. Mater. 14, 1319 (2005).

[17] R. J. Narayan and D. Scholvin, Nanostructured carbon-metal composite films, J. Vac. Sci. Technol. B 23, 1041 (2005).

[18] R. Hauert, A review of modified DLC coatings for biological applications, Diamond Relat. Mater. 12, 583 (2003).

[19] www.plasmoteg.org.by/development_h8_eng.php

[20] D. Weller, H. Brändle, G. Gorman, C.-J. Lin, and H. Notarys, Magnetic and magneto-optical properties of cobalt-platinum alloys with perpendicular magnetic anisotropy, Appl. Phys. Lett. 61, 2726 (1992).

[21] M. Yu, Y. Liu, and D. J. Sellmyer, Nanostructure and magnetic properties of composite CoPt:C films for extremely high-density recording, J. Appl. Phys. 87, 6959 (2000).

[22] S. Sun, C. B. Murray, D. Weller, L. Folks, and A. Moser, Monodisperse $\mathrm{FePt}$ nanoparticles and ferromagnetic FePt nanocrystal superlattices, Science 287, 1989 (2000).

[23] Y. H. Huang, Y. Zhang, G. C. Hadjipanayis, and D. Weller, Fabrication and characterization of ordered FePt nanoparticles, J. Appl. Phys. 93, 7172 (2003). 
[24] Y. Zhang, J. Wan, M. J. Bonder, G. C. Hadjipanayis, and D. Weller, Formation of FePt nanoparticles in annealed FePt/C multilayers, J. Appl. Phys. 93, 7175 (2003).

[25] Y. Xu, M. L. Yan, J. Zhou, and D. J. Sellmyer, Magnetic properties of dilute FePt:C nanocluster films, J. Appl. Phys. 97, 10J320 (2005).

[26] www.nanofilm.com.sg/eng/case_media.htm

[27] D. Liu, G. Benstetter, E. Lodermeier, X. Chen, J. Ding, Y. Liu, J. Zhang, and T. Ma, Surface and structural properties of ultrathin diamond-like carbon coatings, Diamond Relat. Mater. 12, 1594 (2003).

[28] S. J. Dikshit, P. Lele, S. B. Ogale, and S. T. Kshirsagar, Influence of inprocess copper incorporation on the quality of diamond-like films deposited by pulsed laser deposition technique, J. Mater. Res. 11, 2236 (1996).

[29] N. Benchikh, F. Garrelie, C. Donnet, B. Bouchet-Fabre, K. Wolski, F. Rogemond, A. S. Loir, and J. L. Subtil, Nickel-incorporated amorphous carbon film deposited by femtosecond pulsed laser ablation, Thin Sol. Films 482, 287 (2005).

[30] Y.-H. Xu, J.-M. Qiu, J. Bai, J. H. Judy, and J.-P. Wang, FeCo nanoparticles assembled film, J. Appl. Phys. 97, 10J305 (2005).

[31] J. S. Chen, S. P. Lau, G. Y. Chen, Z. Sun, Y. J. Li, B. K. Tay, and J. W. Chai, Deposition of iron containing amorphous carbon films by filtered cathodic vacuum arc technique, Diamond Relat. Mater. 10, 2018 (2001).

[32] D.-Y. Wang, K.-W. Weng, and S.-Y. Hwang, Study on metal-doped diamond-like carbon films synthesized by cathodic arc evaporation, Diamond Relat. Mater. 9, 1762 (2000).

[33] D. Babonneau, T. Cabioc'h, A. Naudon, J. C. Girard, and M. F. Denanot, Silver nanoparticles encapsulated in carbon cages obtained by co-sputtering of the metal and graphite, Surf. Sci. 409, 358 (1998).

[34] K. Schiffmann, M. Fryda, G. Goerigk, R. Lauer, P. Hinze and A. Bulack, Sizes and distances of metal clusters in Au-, Pt-, W- and Fe-containing diamond-like carbon hard coatings: a comparative study by small angle $X$-ray scattering, wide angle $X$-ray diffraction, transmission electron microscopy and scanning tunnelling microscopy, Thin Sol. Films 347, 60 (1999). 
[35] F. C. Fonseca, A. S. Ferlauto, F. Alvarez, G. F. Goya, and R. F. Jardim, Morphological and magnetic properties of carbon-nickel nanocomposite thin films, J. Appl. Phys. 97, 044313 (2005).

[36] V. I. Ivanov-Omskiu, V. I. Siklitskiu, and S. G. Yastrebov, Copper nanoclusters in amorphous hydrogenated carbon, Phys. Sol. State 40, 524 (1998).

[37] Y. H. Lee, T. C. Han, J. C. A. Huang, and C. R. Lin, Analysis of microstructure of magnetic $\mathrm{Fe}_{3} \mathrm{C}$ nanograins embedded in amorphous carbon films, J. Appl. Phys. 94, 1975 (2003).

[38] J.-M. Ting and H. Lee, DLC composite thin films by sputter deposition, Diamond Relat. Mater. 11, 1119 (2002).

[39] S.G. Yastrebov, V. I. Ivanov-Omskiı̌, V. A. Kosobukin, F. Dumitrache, and C. Morosanu, Raman spectra of iron-modified amorphous carbon, Tech. Phys. Lett. 30, 995 (2004).

[40] T. Cabioc'h, A. Naudon, M. Jaouen, D. Thiaudiére, and D. Babonneau, Co-sputtering $\mathrm{C}$-Cu film synthesis: microstructural study of copper precipitates encapsulated into a carbon matrix, Phil. Mag. B 79, 501 (1999).

[41] D. Babonneau, T. Cabioc'h, M.-F. Denanot, and A. Naudon, Microstructural study of a $C$-Fe alloy synthesized by ion-beam sputtering co-deposition, Appl. Phys. Lett. 74, 800 (1999).

[42] D. Babonneau, A. Naudon, T. Cabioc'h, and O. Lyon, Morphology of encapsulated iron nanoparticles obtained by co-sputtering and implantation: a GISAXS study, J. Appl. Cryst. 33, 437 (2000).

[43] D. V. Kulikov, Y. V. Trushin, V. S. Kharlamov, and V. I. Ivanov-Omskiu,, Size distribution of copper nanoclusters in amorphous carbon, Tech. Phys. Lett. 25, 198 (1999).

[44] T. N. Vasilevskaya, N. S. Andreev, I. A. Drozdova, V. N. Filipovich, S. G. Yastrebov, and T. K. Zvonareva, The structure of copper-doped amorphous hydrogenated carbon films, Phys. Sol. State 41, 1918 (1999).

[45] V. I. Ivanov-Omskiı̌, A. B. Lodygin, and S. G. Yastrebov, Analysis of the size-distribution function of metallic nanoclusters in a hydrogenated amorphous carbon host, Semiconductors 36, 743 (2002).

[46] V. I. Ivanov-Omskiŭ, A. V. Kolobov, A. B. Lodygin, and S. G. Yastrebov, Size distribution of cobalt nanoclusters in an amorphous carbon matrix, Semiconductors 38, 1416 (2004). 
[47] S. G. Yastrebov, V. I. Ivanov-Omskiǔ, F. Dumitrache, and C. Morosanu, Raman spectroscopy of amorphous carbon modified with iron, Semiconductors 37, 473 (2003).

[48] C.-C. Chen and F. C.-N. Hong, Structure and properties of diamond-like carbon nanocomposite films containing copper nanoparticles, Appl. Surf. Sci. 242, 261 (2005).

[49] Y. Lee, J. H. Chen, M. S. Guo, G. M. Liu, and C. S. Lue, Structure and magnetic properties of FePt nanograins embedded in amorphous carbon films, J. Magn. Magn. Mater. 282, 15 (2004).

[50] H. Lee and J.-M. Ting, Platiunum-containing diamond-like carbon thin films, J. Am. Ceram. Soc. 87, 2183 (2004).

[51] W.-Y. Wu and J.-M. Ting, Growth and characteristics of carbon films with nano-sized metal particles, Thin Sol. Films 420-421, 166 (2002).

[52] E. Bertran, C. Corbella, A. Pinyol, M. Vives, and J. L. Andújar, Comparative study of metal/amorphous- carbon multilayer structures produced by magnetron sputtering, Diamond Relat. Mater. 12, 1008 (2003).

[53] C. Corbella, M. Vives, A. Pinyol, E. Bertran, C. Canal, M. C. Polo, and J. L. Andújar, Preparation of metal ( $W, M o, N b$, Ti) containing a-C:H films by reactive magnetron sputtering, Surf. Coat. Technol. 177, 409 (2004).

[54] C. Corbella, E. Pascual, G. Oncins, C. Canal, J. L. Andújar, and E. Bertran, Composition and morphology of metal-containing diamond-like carbon films obtained by reactive magnetron sputtering, Thin Sol. Films 482, 293 (2005).

[55] Lexikon der Physik, Bd. 5, Spektrum Akademischer Verlag, Heidelberg Berlin (2000).

[56] G. M. Whitesides and B. Grzybowski, Self-assembly at all scales, Science 295, 2418 (2002).

[57] P. Moriarty, Nanostructured Materials, Rep. Prog. Phys. 64297 (2001), and references therein.

[58] J. C. Jiang, E. I. Meletis, and K. I. Gnanasekar, Self-organized, ordered array of coherent orthogonal column nanostructures in epitaxial $\mathrm{La}_{0.8} \mathrm{Sr}_{0.2} \mathrm{MnO}_{3}$ thin films, Appl. Phys. Lett. 80, 4831 (2002).

[59] D. Babonneau, F. Pailloux, J.-P. Eymery, M.-F. Denanot, Ph. Guérin, E. Fonda, and O. Lyon, Spontaneous organization of columnar nanoparticles in Fe-BN nonocomposite films, Phys. Rev. B 71, 035430 (2005). 
[60] K. B. Blodgett and I. Langmuir, Built-up films of Barium Stearate and their optical properties, Phys. Rev. 51, 964 (1937).

[61] T. Bjørnholm, T. Hassenkam, and N. Reitzel, Supramolecular organization of highly conducting organic thin films by the Langmuir-Blodgett technique, J. Mater. Chem. 9, 1975 (1999).

[62] G. Decher, Fuzzy Nanoassemblies: Toward Layered Polymeric Multicomposites, Science 277, 1232 (1997).

[63] K. Fukunaga, T. Hashimoto, H. Elbs, and G. Krausch, Self-assembly of a lamellar ABC triblock copolymer thin film, Macromolecules 35, 4406 (2002).

[64] R. E. Liesegang, Über einige Eigenschaften von Gallerten, Naturwiss. Wochenschr. 11, 353 (1896).

[65] T. Antal, M. Droz, J. Magnin, and Z. Rácz, Formation of Liesegang patterns: A spinodal decomposition scenario, Phys. Rev. Lett. 83, 2880 (1999).

[66] I. Grosshans, H. Karl, and B. Stritzker, Temporal evolution of ion implanted CdSe distribution in $\mathrm{SiO}_{2}$ on silicon during annealing, Nucl. Instr. Meth. B 219-220, 820 (2004).

[67] http://ujf-grenoble.fr/PHY/AMPposter/ImagesPoster/ imagesPosterDiffrac

[68] F. Richter, Superharte dünne Schichten, Phys. Bl. 52, 355 (1996).

[69] H. Hofsäss, H. Binder, T. Klumpp, and E. Recknagel, Doping and growth of diamond-like carbon films by ion beam deposition, Diamond Relat. Mater. 3, 137 (1993).

[70] C. Ronning, Untersuchungen zum Wachstum und zur Dotierung diamantähnlicher Schichten hergestellt über die Deposition massenseparierter Ionen, Doctoral Thesis, University of Konstanz, Hartung-Gorre Verlag, Konstanz (1996).

[71] C. Ronning, E. Dreher, J.-U. Thiele, P. Oelhafen, and H. Hofsäss, Electronic and atomic structure of undoped and doped ta-C films, Diamond Relat. Mater. 6, 830 (1997).

[72] H. Hofsäss, H. Feldermann, R. Merk, M. Sebastian, and C. Ronning, Cylindrical spike model for the formation of diamondlike thin films by ion deposition, Appl. Phys. A 66, 153 (1998). 
[73] A. C. Ferrari, B. Kleinsorge, N. A. Morrison, A. Hart, V. Stolojan, and J. Robertson, Stress reduction and bond stability during thermal annealing of tetrahedral amorphous carbon, J. Appl. Phys. 85, 7191 (1999).

[74] J. O. Orwa, I. Andrienko, J. L. Peng, S. Prawer, Y. B. Zhang, and S. P. Lau, Thermally induced $s p^{2}$ clustering in tetrahedral amophous carbon (ta-C) films, J. Appl. Phys. 96, 6286 (2004).

[75] M. Chhowalla, Y. Yin, G. A. J. Amaratunga, D. R. McKenzie, and Th. Frauenheim, Highly tetrahedral amorphous carbon films with low stress, Appl. Phys. Lett. 69, 2344 (1996).

[76] T. B. Massalski, H. Okamoto, P. R. Subramanian, and L. Kacprzak (Eds.), Binary Alloy Phase Diagrams, ASM, Metal Park, OH (1996).

[77] H. Föll, Einführung in die Materialwissenschaften 1, Skript (2005). www.tf.uni-kiel.de/matwis/amat/mw1_ge/kap_8/illustr/i8_4_1.html

[78] A. E. W. Jarfors, The influence of carbon on the phases in the coppertitanium system and their precipitation, J. Mater. Sci. 34, 4533 (1999) and references therin.

[79] I. Kararkaya and W. T. Thompson, The Ag-C (silver-carbon) system, Bull. Alloy Phase Diagr. 9, 226 (1988).

[80] R. B. McLellan, The solubility of carbon in solid gold, copper, and silver, Scripta Metallurgica 3, 389 (1969).

[81] G. Mathieu, S. Guiot, and J. Cabané, Solubilite du carbone dans l'argent, le cuivre et l'or, Scripta Metallurgica 7, 421 (1973).

[82] J. Kunze, Nitrogen and carbon in iron and steel, Akademie-Verlag, Berlin (1990).

[83] R. C. Ruhl and M. Cohen, Splat quenching of iron-carbon alloys, Trans. TMS-AIME 245, 241 (1969).

[84] D. L. Williamson, K. Nakazawa, and G. Krauss, A study of the early stages of tempering in an Fe-1.2 pct alloy, Metall. Trans. A 10A, 1351 (1979).

[85] D. H. Jack and K. H. Jack, Carbides and nitrides in steel, Mater. Sci. Eng. 11, 1 (1973).

[86] A. Königer, C. Hammerl, M. Zeitler, and B. Rauschenbach, Formation of metastable iron carbide phases after high-fluence carbon ion implantation into iron at low temperatures, Phys. Rev. B 55, 8143 (1997). 
[87] W. D. Knight, K. Clemenger, W. A. de Heer, W. A. Saunders, M. Y. Chou, and M. L. Cohen, Electronic shell structure and abundances of Sodium clusters, Phys. Rev. Lett. 52, 2141 (1984).

[88] K. Clemenger, Ellipsoidal shell structure in free-electron metal clusters, Phys. Rev. B 32, 1359 (1985).

[89] W. Eckardt and Z. Penzar, Self-consistent Sommerfeld droplet as a simple model for an accurate prediction of the electronic properties of small metal particles, Phys. Rev. B 38, 4273 (1988).

[90] G. Lauritsch, P.-G. Reinhard, J. Meyer, and M. Brack, Triaxially deformed sodium clusters in a self-consistent microscopic description, Phys. Lett. A 160, 179 (1991).

[91] W. A. de Heer, The physics of simple metal clusters: expermiental aspects and simple models, Rev. Mod. Phys. 65, 611 (1993).

[92] M. Brack, The physics of simple metal clusters: self-consistent jellium model and semicalssical approaches, Rev. Mod. Phys. 65, 677 (1993).

[93] B. J. Winter, E. K. Parks, and S. J. Riley, Copper clusters: The interplay between electronic and geometrical structure, J. Chem. Phys. 94, 8618 (1991).

[94] R. Fournier, Theoretical study of the structure of silver clusters, J. Chem. Phys. 115, 2165 (2001).

[95] S. J. Chey, L. Huang, and J. H. Weaver, Interface bonding and manipulation of $\mathrm{Ag}$ and $\mathrm{Cu}$ nanocrystals on Si(111)-(7×7)-based surfaces, Phys. Rev. B 59, 16033 (1999).

[96] K. Reichelt, Nucleation and growth of thin films, Vacuum 38, 1083 (1988).

[97] H. Lüth, Surfaces and interfaces of solids, Springer Series in Surface Sciences 15, Springer Verlag, Berlin Heidelberg (1993).

[98] S. Xu and B. L. Evans, Nucleation and growth of ion beam sputtered metal films, J. Mater. Sci. 27, 3108 (1992).

[99] B. L. Evans, A. I. Maaroof, and S. Xu, Nucleation and growth of platinum and nickel films on amorphous carbon substrates, J. Appl. Phys. 76, 900 (1994).

[100] S. Xu, B. L. Evans, D. I. Flynn, and C. En, The study of island growth of ion beam sputtered metal films by digital image processing, Thin Sol. Films 238, 54 (1994). 
[101] C. Borchers, P. Ricardo, and C. Michaelsen, Interfacial wetting and percolation threshold in ultrathin Ni/C multilayer films, Phil. Mag. A 80, 1669 (2000).

[102] C. Borchers and C. Michaelsen, Ultrathin metal/light material multilayer films: thermodynamics and microstructure, Phil. Mag. A 82, 1195 (2002).

[103] M. Bilaniuk and J. M. Howe, Wetting, bonding and interfacial energy of nanocrystalline metal particles on crystalline DCH polymer and amorphous carbon substrates, Interface Sci. 6, 317 (1998).

[104] L. Z. Mezey and J. Giber, The surface free energies of solid chemical elements: calculation from internal free enthalpies of atomization, Jap. J. Appl. Phys. 21, 1569 (1982).

[105] H. Baumann and K. Bethge, The Frankfurth PIG ion source, Nucl. Instr. Meth. 189, 107 (1981).

[106] G. Sidenius, The high temperature hollow cathode ion source, Nucl. Instr. Meth. 38, 19 (1965).

[107] M. A. Liebermann and A. J. Lichtenberg, Principles of plasma discharges and materials processing, John Wiley \& Sons, New York (1994).

[108] M. Mišina, L. R. Shaginyan, M. Maček, and P. Panjan, Energy resolved ion mass spectroscopy of the plasma during reactive magnetron sputtering, Surf. Coat. Technol. 142-144, 348 (2001).

[109] D. Babonneau, I. R. Videnović, M. G. Garnier, and P. Oelhafen, Morphology and size distribution of gold nanoclusters in $a-C: H$ films studied by grazing incidence small-angle X-ray scattering, Phys. Rev. B 63, 195401 (2001).

[110] Y. Lifshitz, S. R. Kasi, and J. W. Rabalais, Subplantation model for film growth from hyperthermal species: Application to diamond, Phys. Rev. Lett. 62, 1290 (1989).

[111] Y. Lifshitz, S. R. Kasi, J. W. Rabalais, and W. Eckstein, Subplantation model for film growth from hyperthermal species, Phys. Rev. B 41, 10468 (1990).

[112] J. F. Ziegler, J. P. Biersack, and U. Littmark, The stopping and range of ions in solids, Pergamon Press, New York (1985). Free download: www.srim.org 
[113] P. Neumaier, G. Dollinger, A. Bergmaier, W. Eckstein, R. Fischer, L. Görgens, H. Hofsäss, H. U. Jäger, H. Kröger, and C. Ronning, to be published.

[114] C. Ronning, I. Gerhards, M. Seibt, H. Hofsäss, W. Y. Wu, and J. M. Ting, Self-assembled nano-scale multilayer formation using physical vapor deposition methods, Nucl. Instr. Meth. B 242, 261 (2006).

[115] T. K. Monsees, K. Barth, S. Tippelt, K. Heidel, A. Gorbunov, W. Pompe, and R. H. W. Funk, Effects of different titanium alloys and nanosize surface patterning on adhesion, differentiation, and orientation of osteoblastlike cells, Cells Tiss. Org. 180, 81 (2005).

[116] K. Vanormelingen, B. Degroote, H. Pattyn, and A. Vantomme, Thin film growth using hyperthermal ions: a surface morphology study, Surf. Sci. 561, 147 (2004).

[117] N. Tsubouchi and Y. Horino, Low-temperature epitaxial Ni silicidation: The role of hyperthermal species, J. Chem. Phys. 122, 214704 (2005).

[118] C. Weissmantel in K. J. Klabunde (ed.), Thin films from free atoms and particles, Academic Press, Inc., Orlando, (1985).

[119] S. Eyhusen, I. Gerhards, H. Hofsäss, C. Ronning, M. Bomenhofer, J. Zweck, and M. Seibt, Ion energy thresholds and stability of cubic boron nitride, Diamond Relat. Mater. 12, 1877 (2003).

[120] H. Hofsäss, S. Eyhusen, and C. Ronning, On the mechanisms of cubic boron nitride film growth, Diamond Relat. Mater. 13, 1103 (2004).

[121] J. C. Slater, The effects of radiation on materials, J. Appl. Phys. 22, 237 (1951).

[122] F. Seitz and J. S. Koehler, Displacement of atoms during irradiation, Solid State Phys. 2, 305 (1956).

[123] M. Nastasi, J. W. Mayer, and J. K. Hirvonen, Ion-Solid interactions, fundamentals and applications, Cambridge Solid State Science Series, Cambridge University Press, Cambridge (1996).

[124] G. H. Vineyard, Thermal spikes and activated processes, Rad. Eff. 29, 245 (1976).

[125] R. Behrisch (Ed.), Sputtering by particle bombardment I, Topics in Applied Physics 47, Springer Verlag, Heidelberg (1981). 
[126] R. Behrisch (Ed.), Sputtering by particle bombardment II, Topics in Applied Physics 52, Springer Verlag, Heidelberg (1983).

[127] R. Behrisch and K. Wittmaack (Eds.), Sputtering by particle bombardment III, Topics in Applied Physics 64, Springer Verlag, Heidelberg (1991).

[128] H. Kröger, C. Ronning, H. Hofsäss, P. Neumaier, A. Bergmaier, L. Görgens, and G. Dollinger, Diffusion in diamond-like carbon, Diamond Relat. Mater. 12, 2042 (2003).

[129] R. S. Robinson and S. M. Rossnagel in O. Auciello and R. Kelly (eds.), Ion bombardment modification of surfaces, fundamentals and applications, Elsevier Science Publishers B. V., Amsteram (1984).

[130] M. A. Makeev and A.-L. Barabási, Ion-induced effective surface diffusion in ion sputtering, Appl. Phys. Lett. 71, 2800 (1997).

[131] D. B. Williams and C. B. Carter, Transmission electron microscopy, parts I-IV, Plenum Press, New York London (1996).

[132] L. A. Geannuzzi and F. A. Stevie (Eds.), Introduction to focused ion beams, instrumentation, theory, techniques amd practice, Springer Science+Business Media, New York (2005).

[133] L. R. Doolittle, A Semiautomatic Algorithm for Rutherford Backscattering Analysis, Nucl. Instr. Meth. B 15, 227 (1986).

[134] N. P. Barradas, C. Jeynes, and R. P. Wepp, Simulated annealing analysis of Rutherford backscattering data, Appl. Phys. Lett. 71, 291 (1997).

[135] G. Schatz and A. Weidinger, Nuclear condensed matter physics: nuclear methods and applications, Wiley, Chichester (1996).

[136] M. Uhrmacher, K. Pampus, F. J. Bergmeister, D. Purschke, and K. P. Lieb, Energy calibration of the $500 \mathrm{kV}$ heavy ion implanter IoNAs, Nucl. Instr. Meth. B 9, 234 (1985).

[137] A. G. Smekal, Zur Quantentheorie der Dispersion, Naturwiss. 11, 873 (1923).

[138] C. V. Raman and K. S. Krishnan, A new type of secondary radiation, Nature 121, 501 (1928).

[139] D. J. Gardiner, P. R. Graves (Eds.), Practical Raman spectroscopy, Springer Verlag, Berlin Heidelberg (1989). 
[140] P. V. Huong, Structural studies of diamond films and ultrahard materials by Raman and micro-Raman spectroscopies, Diamond Relat. Mater. 1, 33 (1991).

[141] A. C. Ferrari and J. Robertson, Interpretation of Raman spectra of disordered and amorphous carbon, Phys. Rev. B 61, 14095 (2000).

[142] K. W. R. Gilkes, S. Prawer, K. W. Nugent, J. Robertson, H. S. Sands, Y. Lifshitz, and X. Shi, Direct quantitative detection of the $s p^{3}$ bonding in diamond-like carbon films using ultraviolet and visible Raman spectroscopy, J. Appl. Phys. 87, 7283 (2000).

[143] D. G. McCulloch, S. Prawer, and A. Hoffman, Structural investigation of xenon-ion-beam-irradiated glassy carbon, Phys. Rev. B 50, 5905 (1994).

[144] S. Prawer, K. W. Nugent, Y. Lifshitz, G. D. Lempert, E. Grossman, J. Kulik, I. Avigal, and R. Kalish, Systematic variation of the Raman spectra of DLC films as a function of $s p^{2}: s p^{3}$ composition, Diamond Relat. Mater. 5, 433 (1996).

[145] B. D. Cullity, Elements of X-Ray Diffraction, Addison-Wesley Publishing Company, Reading, MA (1978).

[146] Bruker Analytical X-Ray Systems, D8 X-Ray Diffractometer, User's Manual, Karlsruhe (1998).

[147] I. Gerhards, Synthese und Charakterisierung von Metall-Nanoclustern in amorphem Kohlenstoff, Diplomarbeit, 2. Physikalisches Institut, Universität Göttingen (2001).

[148] I. Gerhards, C. Ronning, U. Vetter, H. Hofsäss, H. Gibhardt, G. Eckold, Q. Li, S. T. Lee, Y. L. Huang, and M. Seibt, Ion beam synthesis of amorphous carbon thin films containing metallic nanoclusters, Surf. Coat. Technol. 158-159, 114 (2002).

[149] W.-Y. Wu, private communication (2005).

[150] S. Puri and K. Binder, Surface-directed physe separation with off-critical composition: Analytical and numerical results, Phys. Rev. E 66, 061602 (2002).

[151] K. Yaldram and K. Binder, Unmixing of binary alloys by a vacancy mechanism of diffusion: a computer simulation, Z. Phys. B 82, 405 (1991).

[152] I. Gerhards, H. Stillrich, C. Ronning, H. Hofsäss, and M. Seibt, Selforganized nanoscale multilayer growth in hyperthermal ion deposition, Phys. Rev. B 70, 245418 (2004). 
[153] B. Diu, C. Guthmann, D. Lederer, and B. Roulet, Grundlagen der Statistischen Physik, de Gruyter, Berlin (1994), pp. 96-106.

[154] W. A. Saunders, Fission and liquid-drop behavior of charged gold clusters, Phys. Rev. Lett. 64, 3046 (1990).

[155] M. Niederdrenk, Herstellung, Charakterisierung und Korrosionsverhalten von Eisennitridschichten, Diplomarbeit, 2. Physikalisches Institut, Universität Göttingen (1994).

[156] H. Zutz, Wachstum von Kohlenstoff-Metall-Nanokompositschichten, Diplomarbeit, 2. Physikalisches Institut, Universität Göttingen (2005).

[157] W.-Y. Wu, private communication (2006). 


\section{Acknowledgements}

Ich danke meinem Doktorvater Prof. Dr. Hans Hofsäss für die Möglichkeit, das interessante Thema meiner Diplomarbeit in dieser Doktorarbeit weiterführen zu können. Ich danke ihm für die lange Diskussion der Multilagenbildung und wie man sie am besten anderen Menschen erklärt. Außerdem muss ich ihm für die moralische Unterstützung und die vielen technischen Hilfestellungen bei der etwas problematische Wiederbelebung unseres Mr. Stringer danken.

PD Dr. Michael Seibt danke ich zum einen dafür, dass er sich bereit erklärt hat, das Koreferat dieser Arbeit zu übernehmen. Vor allem muss ich ihm aber für die viele Hilfe am Elektronenmikroskop und beim Verständnis der Bilder danken. In diesem Zusammenhang geht auch ein Dank an seine Mitarbeiter Tore Niermann, Karsten Thiel und Carsten Rudolph.

PD Dr. Carsten Ronning möchte ich für viele Diskussionen physikalischer, technischer oder auch mal kulinarischer Natur danken und für alles, was er mir im Laufe der Jahre beigebracht hat.

Mein besonderer Dank gilt Hayo Zutz. Ich glaube, ohne ihn würde Mr. Stringer immer noch nicht wieder schöne Metall-Kohlenstoffproben herstellen.

Holger Stillrich danke ich für die Einweisung ins Arbeiten an Mr. Stringer und all die Ferndiagnosen und Online-Beratung.

Ich bedanke mich bei Jan Zennek und Holger Gibhardt, an deren Spektrometern die Ramanmessungen durchgeführt wurden; zudem danke ich Matthias Hahn und Volker Radisch für die Präparation meiner letzten drei TEM-Proben am Focussed Ion Beam. Ich danke Velimir Milinovic und Kun Zhang für die Einweisung in die Rutherford Rückstreuungsspektroskopie an IonAs, Ettore Carpene und Salvatore Cusenza für die Hilfe bei Röntgendiffraktometriemessungen.

Daniel Schwen und Sören Eyhusen danke ich für die Hilfe mit all meinen RechnerProblemen.

Danke Detlef Purschke und Andy King, Uwe Ronsöhr, "Pumpen Peter" Arnsberger und Holger Schebela, für die jeweiligen Beiträge die Ihr in Eurer Arbeit am IONAS, mit der Bereitstellung von flüssigem Stickstoff, der Kontrolle der Gase und den Diskussionen über Vakuumpumpen oder Vakuumlecks zu meiner Arbeit geleistet habt.

Zu besonderem Dank bin ich den Mitarbeitern der Werkstätten des 2. Physikalischen Instituts unter Leitung von Klaus Arndt und Kurt Schemmerling und der Zen- 
tralwerstatt der Fakultät verpflichtet. Die vielen Reparaturen und Neuanfertigungen waren immer tadellos!

In allen Lagen des Verwaltungslebens waren Frau Wohlfarth, Lucie Hamdie und Heike Ahrens immer zur Stelle. Vielen Dank! Lucie danke ich auch besonders für das Korrekturlesen dieser Arbeit.

Allen Nudelessern, Daniel, Daniel, Gregor, Hayo, Anne, Sven, Dennis, Frank und Wilma danke ich dafür, dass sie diese schöne Tradition mitgeholfen haben zu begründen und zu bewahren. Bitte lasst sie nicht sterben, wenn meine Küche eines Tages weit weg ist!

Lieber Helge, vielen Dank für die viele Schokolade und die sauren Zungen, die es bei Therapiesitzungen in Deinem Büro immer als Heilmittel gab.

Mein tiefster Dank geht an meine Familie, meine Eltern und Großeltern und an meinen Mann Volker, für ihre fortwährende Unterstützung und Liebe. 


\section{Lebenslauf}

Name: $\quad$ Inga Karolin Hannstein, geb. Gerhards

Addresse: $\quad$ Nonnenstieg 39

37075 Göttingen

Geburtsdatum: 19. Juli 1976

Geburtsort: Brake (Unterweser)

Familienstand: verheiratet

Werdegang

Schule

1982 - 1986 Grundschule Stadland/Jade in Rodenkirchen

1986 - 1988 Orientierungsstufe Stadland

1988 - 1992 Gymnasium Nordenham

1992 - 1993 Caledonia Senior Secondary School, Terrace, B.C., Canada

1993 - 1995 Gymnasium Nordenham

$1995 \quad$ Abitur

Universität

1995 - 1999 Studium der Physik and der Technischen Universität Braunschweig 1999 - 2001 Studium der Physik an der Universität Göttingen

2001 Diplom in Physik. Diplomarbeit unter der Betreuung von Prof. H. Hofsäss.

Titel der Diplomarbeit "Synthese und Charakterisierung von Metall-

Nanoclustern in amorphem Kohlenstoff".

Seit Nov. 2001: Wissenschaftliche Angestellte und Doktorandin unter Betreuung von Prof. H. Hofsäss am II. Physikalischen Institut der Universität Göttingen. 